\title{
On the optimal control of passive or non-expansive systems
}

\author{
Timothy H. Hughes
}

\begin{abstract}
The positive-real and bounded-real lemmas solve two important linear-quadratic optimal control problems for passive and non-expansive systems, respectively. The lemmas assume controllability, yet a passive or non-expansive system can be uncontrollable. In this paper, we solve these optimal control problems without making any assumptions. In particular, we show how to extract the greatest possible amount of energy from a passive but not necessarily controllable system (e.g., a passive electric circuit) using state feedback. A complete characterisation of the set of solutions to the linear matrix inequalities in the positive-real and bounded-real lemmas is also obtained. In addition, we obtain necessary and sufficient conditions for a system to be non-expansive that augment the bounded-real condition with new conditions relevant to uncontrollable systems.
\end{abstract}

Index Terms -Passive, non-expansive, optimal control, positivereal, bounded-real, controllability, observability.

\section{INTRODUCTION}

The positive-real and bounded-real lemmas are recognised as two of the most fundamental results in systems and control. They relate to two important optimal control problems, for passive and non-expansive systems, respectively [1]-[4]. In the positive-real lemma, the solution to the optimal control problem gives the least upper bound on the energy that can be extracted from a passive system. The lemmas also provide results on the solutions of important classes of Linear Matrix Inequalities (LMIs) and Algebraic Riccati Equations (AREs), the theory of spectral factorization, and the concepts of positive-real and bounded-real functions. But the classical versions of these lemmas consider only controllable systems.

In [5], it was emphasised that a passive system (e.g., a passive electric circuit) can be uncontrollable, and a theory of passive linear systems was developed that does not assume controllability. In contrast to other papers on this subject (see [6. Section 3.3] and the discussion following Theorem 13 in the present paper), [5] did not introduce any alternative assumptions. But it did not consider the related optimal control problem, nor did it consider non-expansive systems. It is the purpose of this paper to solve the optimal control problems considered in the positive-real and bounded-real lemmas in the absence of any assumptions. In so doing, we characterise the set of solutions to the LMIs in these two lemmas, and we show how to use state feedback to extract the greatest amount of energy from a passive (not necessarily controllable) linear system. Also, in contrast with the case of controllable systems,

Timothy H. Hughes is with the College of Engineering, Mathematics and Physical Sciences, University of Exeter, Penryn Campus, Penryn, Cornwall, TR10 9EZ, UK, t.h.hughes@exeter.ac.uk we show that for there to exist a solution to the LMI in the bounded-real lemma, and for a system to be non-expansive, it is necessary but not sufficient for the $\mathcal{H}_{\infty}$ norm of the system's transfer function to be bounded above by one. We also provide a necessary and sufficient condition, by introducing two new conditions relevant to uncontrollable systems.

The paper is structured as follows. Section II introduces the notation, and contains preliminary system theoretic results that are formalised using the behavioral approach [7]. In Section III, we review the classical positive-real lemma and the associated optimal control problem. We then state the main results concerning this optimal control problem in Theorems 10, 12 and 13, which are proved in Sections IV] and V] The theorems explicitly characterise the solution to the optimal control problem in terms of an ARE (relevant when the transfer function $H$ satisfies $\lim _{\xi \rightarrow \infty}\left(H(\xi)+H(-\xi)^{T}\right)>0$ ), and a spectral factorization of $H(\xi)+H(-\xi)^{T}$ (relevant in the general case). Section $\nabla I$ contains analogous results relevant to non-expansive systems (Theorems 20, 22, and 23, which are proved in Sections VII and VIII). In particular, we define the new concept of a bounded-real pair of polynomial matrices, which appears in our new necessary and sufficient condition for a system to be non-expansive. Finally, some intermediate results are provided in Appendices $\mathrm{A}, \mathrm{D}$.

\section{NOTATION AND PRELIMINARIES}

The notation in the paper is as follows. $\mathbb{R}(\mathbb{C})$ denotes the real (complex) numbers; $\mathbb{C}_{+}\left(\overline{\mathbb{C}}_{+}\right)$denotes the open (closed) right-half plane; $\mathbb{C}_{-}\left(\overline{\mathbb{C}}_{-}\right)$denotes the open (closed) left-half plane. If $\lambda \in \mathbb{C}$, then $\Re(\lambda)(\Im(\lambda))$ denotes its real (imaginary) part, and $\bar{\lambda}$ its complex conjugate. $\mathbb{R}[\xi](\mathbb{R}(\xi))$ denotes the polynomials (rational functions) in the indeterminate $\xi$ with real coefficients. $\mathbb{R}^{m \times n}$ (resp., $\mathbb{C}^{m \times n}, \mathbb{R}^{m \times n}[\xi], \mathbb{R}^{m \times n}(\xi)$ ) denotes the $m \times n$ matrices with entries from $\mathbb{R}$ (resp., $\mathbb{C}$, $\mathbb{R}[\xi], \mathbb{R}(\xi)$ ). If $H \in \mathbb{R}^{m \times n}, \mathbb{C}^{m \times n}, \mathbb{R}^{m \times n}[\xi]$, or $\mathbb{R}^{m \times n}(\xi)$, then $H^{T}$ denotes its transpose, and if $H$ is nonsingular (i.e., $\operatorname{det}(H) \neq 0$ ) then $H^{-1}$ denotes its inverse. $\mathbb{R}_{s}^{n \times n}$ denotes the real $n \times n$ symmetric matrices. The block column (block diagonal) matrix with entries $H_{1}, \ldots, H_{n}$ is denoted $\operatorname{col}\left(H_{1} \cdots H_{n}\right)\left(\operatorname{diag}\left(H_{1} \cdots H_{n}\right)\right)$. We will use horizontal and vertical lines to indicate the partition in block matrix equations (e.g., see (43)). If $M \in \mathbb{C}^{m \times m}$, then $M>0$ $(M \geq 0)$ indicates that $M$ is Hermitian positive (non-negative) definite, and $\operatorname{spec}(M):=\{\lambda \in \mathbb{C} \mid \operatorname{det}(\lambda I-M)=0\}$.

A $V \in \mathbb{R}^{n \times n}[\xi]$ is called unimodular if its determinant is a non-zero constant (equivalently, $V^{-1} \in \mathbb{R}^{n \times n}[\xi]$ ). The matrices $P \in \mathbb{R}^{m \times n}[\xi]$ and $Q \in \mathbb{R}^{m \times q}[\xi]$ are called left coprime if $\operatorname{rank}\left(\left[\begin{array}{ll}P & -Q\end{array}\right](\lambda)\right)$ is the same for all $\lambda \in \mathbb{C}$. 
If $H \in \mathbb{R}^{m \times n}(\xi)$, then (i) $H^{\star}(\xi):=H(-\xi)^{T}$; (ii) normalrank $(H):=\max _{\lambda \in \mathbb{C}}(\operatorname{rank}(H(\lambda)))$; and (iii) $H$ is called proper if $\lim _{\xi \rightarrow \infty}(H(\xi))$ exists, and strictly proper if $\lim _{\xi \rightarrow \infty}(H(\xi))=0$. If $Z \in \mathbb{R}^{r \times n}(\xi)$ and $H:=Z^{\star} Z$, then $Z$ is called a spectral factor of $H$ if (i) $Z$ is analytic in $\mathbb{C}_{+}$; and (ii) $Z(\lambda)$ has full row rank for all $\lambda \in \mathbb{C}_{+}$. If $H \in \mathbb{R}^{m \times n}(\xi)$, then $\|H\|_{\infty}$ denotes its $\mathscr{H}_{\infty}$ norm, and it is called boundedreal if $\|H\|_{\infty} \leq 1$ (i.e., $H$ satisfies $I-H(\bar{\lambda})^{T} H(\lambda) \geq 0$ for all $\lambda \in \overline{\mathbb{C}}_{+}$). If $m=n$, then $H$ is called positive-real if (i) $H$ is analytic in $\mathbb{C}_{+}$; and (ii) $H(\bar{\lambda})^{T}+H(\lambda) \geq 0$ for all $\lambda \in \mathbb{C}_{+}$.

We let $\mathcal{L}_{2}^{\text {loc }}\left(\mathbb{R}, \mathbb{R}^{k}\right)$ denote the ( $k$-vector-valued) locally square integrable functions, and if $\mathbf{w} \in \mathcal{L}_{2}^{\text {loc }}\left(\mathbb{R}, \mathbb{R}^{k}\right)$ then $\mathbf{w}^{T}$ denotes the function satisfying $\mathbf{w}^{T}(t)=\mathbf{w}(t)^{T}$ for all $t \in \mathbb{R}$.

We will consider state-space systems of the form

$$
\begin{gathered}
\mathcal{B}_{s}=\left\{(\mathbf{u}, \mathbf{y}, \mathbf{x}) \in \mathcal{L}_{2}^{\text {loc }}\left(\mathbb{R}, \mathbb{R}^{n}\right) \times \mathcal{L}_{2}^{\text {loc }}\left(\mathbb{R}, \mathbb{R}^{m}\right) \times \mathcal{L}_{2}^{\text {loc }}\left(\mathbb{R}, \mathbb{R}^{d}\right)\right. \\
\text { such that } \left.\frac{d \mathbf{x}}{d t}=A \mathbf{x}+B \mathbf{u} \text { and } \mathbf{y}=C \mathbf{x}+D \mathbf{u}\right\},
\end{gathered}
$$$$
\text { with } A \in \mathbb{R}^{d \times d}, B \in \mathbb{R}^{d \times n}, C \in \mathbb{R}^{m \times d} \text { and } D \in \mathbb{R}^{m \times n} \text {, }
$$

and we interpret differentiation in a weak sense (see [7, Section 2.3.2]). In particular, for any given $\mathbf{u} \in \mathcal{L}_{2}^{\text {loc }}\left(\mathbb{R}, \mathbb{R}^{n}\right)$ and $\mathbf{x}_{0} \in$ $\mathbb{R}^{d}$, there exists $(\mathbf{u}, \mathbf{y}, \mathbf{x}) \in \mathcal{B}_{s}$ such that

$$
\begin{aligned}
& \mathbf{x}(t)=e^{A\left(t-t_{0}\right)} \mathbf{x}_{0}+\int_{t_{0}}^{t} e^{A(t-\tau)} B \mathbf{u}(\tau) d \tau, \text { and } \\
& \mathbf{y}(t)=C e^{A\left(t-t_{0}\right)} \mathbf{x}_{0}+D \mathbf{u}(t)+\int_{t_{0}}^{t} C e^{A(t-\tau)} B \mathbf{u}(\tau) d \tau,
\end{aligned}
$$

for all $t \geq t_{0}$. Moreover, if $(\mathbf{u}, \mathbf{y}, \mathbf{x}) \in \mathcal{B}_{s}$, then there exists $\mathbf{x}_{0} \in \mathbb{R}^{d}$ such that $\mathbf{x}$ satisfies (2) for (almost) all $t \geq t_{0}$, so $\mathbf{x}(t)$ is determined by (2) in this interval (and $\mathbf{x}\left(t_{0}\right)=\mathbf{x}_{0}$ ).

The external behavior of (1) is denoted by

$$
\mathcal{B}_{s}^{(\mathbf{u}, \mathbf{y})}:=\left\{(\mathbf{u}, \mathbf{y}) \mid \exists \mathbf{x} \text { such that }(\mathbf{u}, \mathbf{y}, \mathbf{x}) \in \mathcal{B}_{s}\right\},
$$

and has the properties outlined in the following two lemmas, which are easily shown from results in [8].

Lemma 1: Let $\mathcal{B}_{s}$ be as in (1) and $\mathcal{A}(\xi):=\xi I-A$. There exist polynomial matrices $P, Q, M, N, U, V, E, F, G$ such that 1. $\left[\begin{array}{cc}M & N \\ U & V\end{array}\right]\left[\begin{array}{ccc}-D & I & -C \\ -B & 0 & \mathcal{A}\end{array}\right]=\left[\begin{array}{ccc}-P & Q & 0 \\ -E & -F & G\end{array}\right]$

2. $\left[\begin{array}{cc}M & N \\ U & V\end{array}\right]$ is unimodular; and

3. $G$ is nonsingular.

Furthermore, whenever conditions 13 are satisfied, then the external behavior $\mathcal{B}_{s}^{(\mathbf{u}, \mathbf{y})}$ in (4) satisfies $\mathcal{B}_{s}^{(\mathbf{u}, \mathbf{y})}=\mathcal{B}$, where

$\mathcal{B}=\left\{(\mathbf{u}, \mathbf{y}) \in \mathcal{L}_{2}^{\text {loc }}\left(\mathbb{R}, \mathbb{R}^{n}\right) \times \mathcal{L}_{2}^{\text {loc }}\left(\mathbb{R}, \mathbb{R}^{m}\right) \mid P\left(\frac{d}{d t}\right) \mathbf{u}=Q\left(\frac{d}{d t}\right) \mathbf{y}\right\}$

and we call $(A, B, C, D)$ a realization of $(P, Q)$.

Lemma 2: Let $\mathcal{B}$ be as in (5) with $P \in \mathbb{R}^{m \times n}[\xi]$ and $Q \in \mathbb{R}^{m \times m}[\xi]$ where $Q$ is nonsingular and $Q^{-1} P$ is proper. Then there exists $\mathcal{B}_{s}$ as in (1) such that $\mathcal{B}=\mathcal{B}_{s}^{(\mathbf{u}, \mathbf{y})}$. Furthermore, whenever $\mathcal{B}_{s}$ in (1) satisfies $\mathcal{B}=\mathcal{B}_{s}^{(\mathbf{u}, \mathbf{y})}$, then there exist polynomial matrices $M, N, U, V, E, F$ and $G$ satisfying conditions 13 of Lemma 1.

Remark 3: If $\mathcal{B}_{s}$ in (1) and $\mathcal{B}$ in (5) satisfy $\mathcal{B}_{s}^{(\mathbf{u}, \mathbf{y})}=\mathcal{B}$, then $H(\xi):=D+C(\xi I-A)^{-1} B$ satisfies $Q^{-1} P=H$. However, the condition $Q^{-1} P=H$ only guarantees that $\mathcal{B}_{s}^{(\mathbf{u}, \mathbf{y})}$ takes the form of (5) if $P$ and $Q$ are left coprime.

A system $\mathcal{B}$ is called controllable if, for any two trajectories $\mathbf{w}_{1}, \mathbf{w}_{2} \in \mathcal{B}$ and $t_{0} \in \mathbb{R}$, there exists $\mathbf{w} \in \mathcal{B}$ and $t_{1} \geq t_{0}$ such that $\mathbf{w}(t)=\mathbf{w}_{1}(t)$ for all $t \leq t_{0}$ and $\mathbf{w}(t)=\mathbf{w}_{2}(t)$ for all $t \geq t_{1}$ [7. Definition 5.2.2]; and stabilizable if for any $\mathbf{w}_{1} \in \mathcal{B}$ there exists $\mathbf{w} \in \mathcal{B}$ such that $\mathbf{w}(t)=\mathbf{w}_{1}(t)$ for all $t \leq t_{0}$ and $\lim _{t \rightarrow \infty} \mathbf{w}(t)=0$ [7, Definition 5.2.29]. The behavior $\mathcal{B}$ in (5) is controllable (resp., stabilizable) if and only if $P$ and $Q$ are left coprime (resp., $\operatorname{rank}\left(\left[\begin{array}{ll}P & -Q\end{array}\right](\lambda)\right)$ is the same for all $\lambda \in \overline{\mathbb{C}}_{+}$) [7, Theorems 5.2.10 and 5.2.30]. We call the pair $(A, B)$ controllable (resp., stabilizable) if $\mathcal{B}_{s}$ is controllable (resp., stabilizable), which holds if and only if $\operatorname{rank}\left(\left[\begin{array}{ll}\lambda I-A & B\end{array}\right]\right)=d$ for all $\lambda \in \mathbb{C}$ (resp., $\lambda \in \overline{\mathbb{C}}_{+}$).

Finally, if $\mathcal{B}_{s}$ takes the form of (1), then we call the pair $(C, A)$ observable if $(\mathbf{u}, \mathbf{y}, \mathbf{x}) \in \mathcal{B}_{s}$ and $(\mathbf{u}, \mathbf{y}, \hat{\mathbf{x}}) \in \mathcal{B}_{s}$ imply $\mathbf{x}=\hat{\mathbf{x}}$ [7, Definition 5.3.2]. If, in addition, $\mathcal{B}_{s}^{(\mathbf{u}, \mathbf{y})}$ takes the form of (5), then we call $(A, B, C, D)$ an observable realization for $(P, Q)$. With the notation

$$
V_{o}:=\operatorname{col}\left(\begin{array}{llll}
C & C A & \cdots & C A^{d-1}
\end{array}\right),
$$

then $(C, A)$ is observable if and only if $\operatorname{rank}\left(V_{o}\right)=d[7$, Theorem 5.3.9].

Remark 4: It is easily shown that if $\mathcal{B}_{s}$ is controllable (resp., stabilizable) then so too is $\mathcal{B}_{s}^{(\mathbf{u}, \mathbf{y})}$. Furthermore, if $(C, A)$ is observable and $\mathcal{B}_{s}^{(\mathbf{u}, \mathbf{y})}$ is controllable (resp., stabilizable), then $\mathcal{B}_{s}$ is controllable (resp., stabilizable).

\section{PASSIVE SYSTEMS}

The positive-real lemma considers the optimal control problem concerning the available energy for a passive system:

Definition 5 (Available energy, Passive system): Let $\mathcal{B}_{s}$ be as in (1) with $m=n$. For any given $\mathbf{x}_{0} \in \mathbb{R}^{d}$, let

$$
\begin{array}{r}
\mathcal{E}_{+}^{\sigma_{p}}\left(\mathbf{x}_{0}\right)=\left\{\int_{t_{0}}^{t_{1}}-\left(\mathbf{u}^{T} \mathbf{y}\right)(t) d t \mid t_{1} \geq t_{0},(\mathbf{u}, \mathbf{y}, \mathbf{x}) \in \mathcal{B}_{s},\right. \\
\text { and } \left.\mathbf{x}\left(t_{0}\right)=\mathbf{x}_{0}\right\} .
\end{array}
$$

Then the available energy $S_{a}^{\sigma_{p}}$ satisfies (i) $S_{a}^{\sigma_{p}}\left(\mathbf{x}_{0}\right)=$ $\sup \left(\mathcal{E}_{+}^{\sigma_{p}}\left(\mathbf{x}_{0}\right)\right)$ if $\mathcal{E}_{+}^{\sigma_{p}}\left(\mathbf{x}_{0}\right)$ is bounded above; and (ii) $S_{a}^{\sigma_{p}}\left(\mathbf{x}_{0}\right)=\infty$ otherwise. If $S_{a}^{\sigma_{p}}\left(\mathbf{x}_{0}\right)<\infty$ for all $\mathbf{x}_{0} \in \mathbb{R}^{d}$, then $\mathcal{B}_{s}^{(\mathbf{u}, \mathbf{y})}$ is called passive.

In words, the available energy is the least upper bound on the energy that can be extracted from $t_{0}$ onwards.

The positive-real lemma provides the solution (if it exists) to the optimal control problem in Definition 5, and several necessary and sufficient conditions for passivity. These relate: (a) the existence of real matrices $X \geq 0$ such that

$$
\Omega(X):=\left[\begin{array}{cc}
-A^{T} X-X A & C^{T}-X B \\
C-B^{T} X & D+D^{T}
\end{array}\right]
$$

satisfies $\Omega(X) \geq 0$; (b) whether the transfer function

$$
H(\xi):=D+C(\xi I-A)^{-1} B
$$

is positive-real; and (c) a second optimal control problem concerning the required energy, defined as follows

Definition 6 (Required energy): Let $\mathcal{B}_{s}$ be as in (1) with $m=n$. For any given $\mathbf{x}_{0} \in \mathbb{R}^{d}$, let

$$
\begin{array}{r}
\mathcal{E}_{-}^{\sigma_{p}}\left(\mathbf{x}_{0}\right)=\left\{\int_{t_{1}}^{t_{0}}\left(\mathbf{u}^{T} \mathbf{y}\right)(t) d t \mid t_{1} \leq t_{0},(\mathbf{u}, \mathbf{y}, \mathbf{x}) \in \mathcal{B}_{s},\right. \\
\left.\mathbf{x}\left(t_{1}\right)=0 \text { and } \mathbf{x}\left(t_{0}\right)=\mathbf{x}_{0}\right\} .
\end{array}
$$


Then the required energy $S_{r}^{\sigma_{p}}$ satisfies (i) $S_{r}^{\sigma_{p}}\left(\mathbf{x}_{0}\right)=$ $\sup \left(\mathcal{E}_{-}^{\sigma_{p}}\left(\mathbf{x}_{0}\right)\right)$ if $\mathcal{E}_{-}^{\sigma_{p}}\left(\mathbf{x}_{0}\right)$ is bounded above; and (ii) $S_{r}^{\sigma_{p}}\left(\mathbf{x}_{0}\right)=\infty$ otherwise.

Also, if $D+D^{T}>0$, then, with the notation

$$
\begin{aligned}
& \Gamma(X):=-A^{T} X-X A \\
& \quad-\left(C^{T}-X B\right)\left(D+D^{T}\right)^{-1}\left(C-B^{T} X\right), \\
& \text { and } A_{\Gamma}(X):=A-B\left(D+D^{T}\right)^{-1}\left(C-B^{T} X\right),
\end{aligned}
$$

the conditions (a)-(c) also relate to the spectral properties of $A_{\Gamma}(X)$ for solutions $X$ to the $\operatorname{ARE} \Gamma(X)=0$. Critically to this paper, it is typically assumed that $(A, B)$ is controllable and $(C, A)$ is observable.

Lemma 7 (Positive-real lemma): Let $\mathcal{B}_{s}$ be as in (1) with $m=n,(A, B)$ controllable and $(C, A)$ observable; let $S_{a}^{\sigma_{p}}$ and $S_{r}^{\sigma_{p}}$ be as in Definitions 5 and 6 and let $\Omega$ and $H$ be as in (7)-(8). The following are equivalent:

1. $S_{a}^{\sigma_{p}}\left(\mathbf{x}_{0}\right)<\infty$ for all $\mathbf{x}_{0} \in \mathbb{R}^{d}$ (i.e., $\mathcal{B}_{s}^{(\mathbf{u}, \mathbf{y})}$ is passive).

2. $S_{r}^{\sigma_{p}}\left(\mathbf{x}_{0}\right)<\infty$ for all $\mathbf{x}_{0} \in \mathbb{R}^{d}$.

3. $H$ is positive-real.

4. There exists $X \in \mathbb{R}_{s}^{d \times d}$ such that $X \geq 0$ and $\Omega(X) \geq 0$.

5. $S_{a}^{\sigma_{p}}\left(\mathbf{x}_{0}\right)=\frac{1}{2} \mathbf{x}_{0}^{T} X_{-} \mathbf{x}_{0}$ and $S_{r}^{\sigma_{p}}\left(\mathbf{x}_{0}\right)=\frac{1}{2} \mathbf{x}_{0}^{T} X_{+} \mathbf{x}_{0}$, where $X_{-}, X_{+} \in \mathbb{R}_{s}^{d \times d}$ satisfy (i) $\Omega\left(X_{-}\right) \geq 0$ and $\Omega\left(X_{+}\right) \geq 0$; and (ii) if $X \in \mathbb{R}_{s}^{d \times d}$ satisfies $\Omega(X) \geq 0$, then $0<X_{-} \leq$ $X \leq X_{+}$.

If, in addition, $D+D^{T}>0$ and $\Gamma(X), A_{\Gamma}(X)$ are as in (9)(10), then $1-5]$ are equivalent to each of the following:

6. There exists a unique $X_{-} \in \mathbb{R}_{s}^{d \times d}$ satisfying (i) $X_{-} \geq 0$;

(ii) $\Gamma\left(X_{-}\right)=0$; and (iii) $\operatorname{spec}\left(A_{\Gamma}\left(X_{-}\right)\right) \in \overline{\mathbb{C}}_{-}$.

7. There exists a unique $X_{+} \in \mathbb{R}_{s}^{d \times d}$ satisfying (i) $X_{+} \geq 0$; (ii) $\Gamma\left(X_{+}\right)=0$; and (iii) $\operatorname{spec}\left(A_{\Gamma}\left(X_{+}\right)\right) \in \overline{\mathbb{C}}_{+}$.

Moreover, if conditions 6 and 7 hold, then $S_{a}^{\sigma_{p}}\left(\mathbf{x}_{0}\right)=$ $\frac{1}{2} \mathbf{x}_{0}^{T} X_{-} \mathbf{x}_{0}$ and $S_{r}^{\sigma_{p}}\left(\mathbf{x}_{0}\right)=\frac{1}{2} \mathbf{x}_{0}^{T} X_{+} \mathbf{x}_{0}$.

Proof: See [2, Sections 3-5].

It was shown in [5] that, if controllability and observability are not assumed, then the positive-real condition is necessary but not sufficient for there to exist a solution to the LMI in the positive-real lemma (condition 4 in Lemma 7). A new condition was provided in terms of the polynomial matrices $P, Q$ describing the external behavior (see Lemma 1). Specifically, it was shown that there exists a solution to the LMI if and only if $(P, Q)$ are a positive-real pair, defined as follows.

Definition 8 (Positive-real pair): Let $P, Q \in \mathbb{R}^{n \times n}[\xi]$. We call $(P, Q)$ a positive-real pair if the following hold:

(a) $P(\lambda) Q(\bar{\lambda})^{T}+Q(\lambda) P(\bar{\lambda})^{T} \geq 0$ for all $\lambda \in \mathbb{C}_{+}$.

(b) $\operatorname{rank}\left(\left[\begin{array}{ll}P & -Q\end{array}\right](\lambda)\right)=n$ for all $\lambda \in \overline{\mathbb{C}}_{+}$.

(c) If $\mathbf{p} \in \mathbb{R}^{n}[\xi]$ and $\lambda \in \mathbb{C}$ satisfy $\mathbf{p}^{T}\left(P Q^{\star}+Q P^{\star}\right)=0$ and $\mathbf{p}(\lambda)^{T}\left[\begin{array}{ll}P & -Q\end{array}\right](\lambda)=0$, then $\mathbf{p}(\lambda)=0$.

Remark 9: If $\mathcal{B}_{s}$ is as in (1), then $\mathcal{B}_{s}^{(\mathbf{u}, \mathbf{y})}$ takes the form indicated in Lemma 1. With $P, Q$ as defined in Lemma $1, Q$ is nonsingular, and $H:=Q^{-1} P$ satisfies (8). Then, condition (a) of Definition 8 is equivalent to $H$ being positive-real [5. Sections 4-5]. Also, condition [b) is equivalent to the stabilizability of $\mathcal{B}_{s}^{(\mathbf{u}, \mathbf{y})}$. Finally, a physical interpretation of condition (c) is given in [5, Sections 4-5]. This condition relates to the fact that, if (i) $(\mathbf{u}, \mathbf{y}, \mathbf{x}) \in \mathcal{B}_{s}$ and $t_{1}>t_{0}$ satisfy $\mathbf{x}\left(t_{0}\right)=\mathbf{x}\left(t_{1}\right)=0$ and $\int_{t_{0}}^{t_{1}}\left(\mathbf{u}^{T} \mathbf{y}\right)(t) d t=0$; (ii)
$(0, \hat{\mathbf{y}}, \hat{\mathbf{x}}) \in \mathcal{B}_{s} ;$ and (iii) $\alpha \in \mathbb{R}$, then $(\alpha \mathbf{u}, \alpha \mathbf{y}+\hat{\mathbf{y}}, \alpha \mathbf{x}+\hat{\mathbf{x}}) \in$ $\mathcal{B}_{s}$. It can then be shown that, if $\mathcal{B}_{s}^{(\mathbf{u}, \mathbf{y})}$ is passive, then $\int_{t_{0}}^{t_{1}}\left(\mathbf{u}^{T} \hat{\mathbf{y}}\right)(t) d t=0$.

In this paper, we develop the results in [5] to solve the optimal control problem of extracting the greatest possible amount of energy from a passive system, and to characterise the set of solutions to the LMI considered in the positive-real lemma, in the absence of any controllability or observability assumptions. The main results in this section are in the next three theorems, which will be proved in Sections IV and $\mathrm{V}$

Theorem 10: Let $\mathcal{B}_{s}$ and $\mathcal{B}_{s}^{(\mathbf{u}, \mathbf{y})}$ be as in (1) and (4) with $m=n$; let $S_{a}^{\sigma_{p}}$ be as in Definition [5, and let $V_{o}$ and $\Omega$ be as in (6) and (7). The following are equivalent:

1. $S_{a}^{\sigma_{p}}\left(\mathbf{x}_{0}\right)<\infty$ for all $\mathbf{x}_{0} \in \mathbb{R}^{d}$ (i.e., $\mathcal{B}_{s}^{(\mathbf{u}, \mathbf{y})}$ is passive).

2. The external behavior $\mathcal{B}_{s}^{(\mathbf{u}, \mathbf{y})}$ takes the form of (5), where $(P, Q)$ is a positive-real pair.

3. There exists $X \in \mathbb{R}_{s}^{d \times d}$ such that $X \geq 0$ and $\Omega(X) \geq 0$.

4. $S_{a}^{\sigma_{p}}\left(\mathbf{x}_{0}\right)=\frac{1}{2} \mathbf{x}_{0}^{T} X_{-} \mathbf{x}_{0}$, where $X_{-} \in \mathbb{R}_{s}^{d \times d}$ satisfies (i) $X_{-} \geq 0$; (ii) $\Omega\left(X_{-}\right) \geq 0$; (iii) if $\mathbf{z} \in \mathbb{R}^{d}$, then $V_{o} \mathbf{z}=$ $0 \Longleftrightarrow X_{-} \mathbf{z}=0$; and (iv) if $X \in \mathbb{R}_{s}^{d \times d}$ satisfies $X \geq 0$ and $\Omega(X) \geq 0$, then $X_{-} \leq X$.

Moreover, if $(C, A)$ is observable and the above conditions hold, then (i) $\operatorname{spec}(A) \in \overline{\mathbb{C}}_{-}$; and (ii) if $X \in \mathbb{R}_{s}^{d \times d}$ satisfies $\Omega(X) \geq 0$, then $X_{-} \leq X$.

Remark 11: We note from Theorem 10 that, for a passive system, $\mathbf{z} \in \mathbb{R}^{d}$ satisfies $V_{o} \mathbf{z}=0$ if and only if $S_{a}^{\sigma_{p}}(\mathbf{z})=0$. In words, the available energy of the state $\mathrm{z}$ is zero if and only if $\mathbf{z}$ is an unobservable mode (we call $\mathbf{z}$ an unobservable mode if $(0,0, \mathbf{x}) \in \mathcal{B}_{s}$ where $\mathbf{x}(t)=e^{A t} \mathbf{z}$ for all $\left.t \in \mathbb{R}\right)$. $\triangle$

The next theorem provides an explicit expression for the available energy for the case with $D+D^{T}>0$.

Theorem 12: Let $\mathcal{B}_{s}$ be as in (1) with $m=n$; let $S_{a}^{\sigma_{p}}$ be as in Definition 5, let $V_{o}, \Gamma$ and $A_{\Gamma}$ be as in (6), (9) and (10), respectively; and let $D+D^{T}>0$. The following are equivalent: 1. $S_{a}^{\sigma_{p}}\left(\mathbf{x}_{0}\right)<\infty$ for all $\mathbf{x}_{0} \in \mathbb{R}^{d}$ (i.e., $\mathcal{B}_{s}^{(\mathbf{u}, \mathbf{y})}$ is passive).

2. There exists $X_{-} \in \mathbb{R}_{s}^{d \times d}$ satisfying (i) $X_{-} \geq 0$; (ii) $\Gamma\left(X_{-}\right)=0$; (iii) if $\mathbf{z} \in \mathbb{R}^{d}$ satisfies $V_{o} \mathbf{z}=0$, then $X_{-} \mathbf{z}=0$; and (iv) if $\lambda \in \mathbb{C}_{+}$and $\mathbf{z} \in \mathbb{C}^{d}$ satisfy $A_{\Gamma}\left(X_{-}\right) \mathbf{z}=\lambda \mathbf{z}$, then $V_{o} \mathbf{z}=0$.

Moreover, if these conditions hold, then $S_{a}^{\sigma_{p}}\left(\mathbf{x}_{0}\right)=\frac{1}{2} \mathbf{x}_{0}^{T} X_{-} \mathbf{x}_{0}$. The final theorem provides an explicit expression for the available energy in the general case.

Theorem 13: Let $\mathcal{B}_{s}$ be as in (1) with $m=n$; let $S_{a}^{\sigma_{p}}$ be as in Definition [5, and let $V_{o}$ and $H$ be as in (6) and (8). The following are equivalent:

1. $S_{a}^{\sigma_{p}}\left(\mathbf{x}_{0}\right)<\infty$ for all $\mathbf{x}_{0} \in \mathbb{R}^{d}$ (i.e., $\mathcal{B}_{s}^{(\mathbf{u}, \mathbf{y})}$ is passive).

2. There exists $X_{-} \in \mathbb{R}_{s}^{d \times d}$ satisfying (i) $X_{-} \geq 0$; (ii) if $\mathbf{z} \in \mathbb{R}^{d}$ satisfies $V_{o} \mathbf{z}=0$, then $X_{-} \mathbf{z}=0$; and (iii) there exist real matrices $L$ and $W$ such that

$$
\begin{aligned}
& \text { (iiia) }-A^{T} X_{-}-X_{-} A=L^{T} L, C-B^{T} X_{-}=W^{T} L \text {, and } \\
& D+D^{T}=W^{T} W \text {; and }
\end{aligned}
$$

Moreover, if these conditions hold, then $S_{a}^{\sigma_{p}}\left(\mathbf{x}_{0}\right)=\frac{1}{2} \mathbf{x}_{0}^{T} X_{-} \mathbf{x}_{0}$.

In proving Theorems 10, 12 and 13, we show how to compute the available energy $S_{a}^{\sigma_{p}}$ and obtain a linear state feed- 
back law such that, with $\mathbf{x}_{0}:=\mathbf{x}\left(t_{0}\right)$, then $\int_{t_{0}}^{\infty}-\left(\mathbf{u}^{T} \mathbf{y}\right)(t) d t$ is arbitrarily close to $S_{a}^{\sigma_{p}}\left(\mathbf{x}_{0}\right)$ (see Remark 16).

We next present an example to illustrate the distinction between the results in this section and other papers in the literature that deal with similar objectives.

It has long been recognised that the controllability and observability assumptions in the positive-real lemma are unduly restrictive, and there have been many notable attempts to relax these assumptions. A comprehensive summary is provided in [6. Section 3.3] (see also [9] for additional properties of the LMI $\Omega(X) \geq 0$ ). These results focus on the equivalence of the positive-real condition with the existence of solutions $X \in \mathbb{R}_{s}^{d \times d}$ to an LMI (similar to condition 3 of Theorem 10) or an ARE (similar to condition 2 of Theorem [12] [10][13]. None of these papers explicitly consider the optimal control problem in Definition 5. Also, each of these papers introduce alternative assumptions that are not necessary for guaranteeing a solution to the optimal control problem. These assumptions include: (i) $\operatorname{spec}(A) \in \mathbb{C}_{-}[12]$, [13]; (ii) $(A, B)$ is stabilizable [10], [11]; (iii) $H+H^{\star}$ is nonsingular [11]; (iv) $H(j \omega)+H(-j \omega)^{T}>0$ for all $\omega \in \mathbb{R}[10]$, [13] (note that this implies (iii)); and (v) $(C, A)$ is observable [10], [11]. A key objective of this paper is to avoid such assumptions entirely.

We also note that several papers have sought to demonstrate the equivalence of the conditions (a) $H(j \omega)+H(-j \omega)^{T} \geq 0$ for all $\omega \in \mathbb{R}$; and (b) there exists $X \in \mathbb{R}_{s}^{d \times d}$ (not necessarily non-negative definite) such that $\Omega(X) \geq 0$ [14], [15]. The papers [16], [17] consider a similar problem using the formalism of the behavioral approach. These papers again introduce additional assumptions. Specifically, [14] assumes that $A$ is unmixed; and [15] assumes sign controllability. Both of these conditions imply the assumption (vi) $[j \omega I-A \quad B]$ has full row rank for all $\omega \in \mathbb{R}$. Also, [17] assumes conditions (iii), (v) and (vi); and [16] considers only single-input single-output systems that satisfy conditions (v) and (vi).

However, there are physical systems that do not satisfy any of the assumptions in these papers. For example, consider the two electric circuits in Fig. 1 Note that, for each of these circuits, the pair $(A, B)$ is not stabilizable. This implies that there is no state feedback law that transfers the internal currents and voltages to zero (however, there is a state feedback law that transfers the external currents and voltages to zero, and so the external behavior $\mathcal{B}_{s}^{(i, v)}$ is stabilizable). Also, both circuits violate assumptions (i), (ii) and (vi) in the previous discussion, and the circuit on the right has $H+H^{\star}=0$ (and so violates assumptions (iii) and (iv)). Now, consider the circuit on the left. Following note A[1, we let

$$
T:=\left[\begin{array}{cccc}
1 & 1 & -1 & -1 \\
0 & 1 & 0 & 0 \\
0 & 0 & 1 & 0 \\
0 & 0 & 0 & 1
\end{array}\right], \text { so } T^{-1}=\left[\begin{array}{cccc}
1 & -1 & 1 & 1 \\
0 & 1 & 0 & 0 \\
0 & 0 & 1 & 0 \\
0 & 0 & 0 & 1
\end{array}\right],
$$

which transform the system into observer staircase form:

$$
\hat{A}:=T A T^{-1}=\left[\begin{array}{cccc}
-1 & 0 & 0 & 0 \\
-1 & 0 & -1 & 0 \\
-1 & 1 & -1 & -1 \\
0 & -1 & 0 & 0
\end{array}\right], \hat{B}:=T B=\left[\begin{array}{l}
0 \\
1 \\
1 \\
1
\end{array}\right]
$$

and $\hat{C}:=C T^{-1}=\left[\begin{array}{llll}-1 & 0 & 0 & 0\end{array}\right]$.

We note that the final three columns of $V_{o} T^{-1}$ are zero (so this circuit also violates assumption (v)), and it follows from Theorem 10 that $X_{-}=T^{T} \hat{X}_{-} T$ where $\hat{X}_{-}=\operatorname{diag}\left(\begin{array}{ll}\lambda & 0\end{array}\right)$ and $\lambda$ is the least real positive number satisfying

$$
\left[\begin{array}{cc}
-\hat{A}^{T} \hat{X}_{-}-\hat{X}_{-} \hat{A} & \hat{C}^{T}-\hat{X}_{-} \hat{B} \\
\hat{C}-\hat{B}^{T} \hat{X}_{-} & D+D^{T}
\end{array}\right]=\left[\begin{array}{ccc}
2 \lambda & 0 & -1 \\
0 & 03 \times 3 & 0 \\
-1 & 0 & 2
\end{array}\right] \geq 0 .
$$

Thus, $\lambda=\frac{1}{4}$, and from Theorem 10 we conclude that, with $\mathbf{x}_{0}=\mathbf{x}(0)$, then $S_{a}^{\sigma_{p}}\left(\mathbf{x}_{0}\right)=\frac{1}{8}\left(\left(i_{1}+i_{2}-v_{3}-v_{4}\right)(0)\right)^{2}$. Note that more energy can be extracted from this system than can be extracted from the system $\mathcal{B}=\left\{(u, y) \in \mathcal{L}_{2}^{\text {loc }}(\mathbb{R}, \mathbb{R}) \times\right.$ $\left.\mathcal{L}_{2}^{\text {loc }}(\mathbb{R}, \mathbb{R}) \mid y=u\right\}$ (for which $\int_{t_{0}}^{t_{1}}-u(t) y(t) d t \leq 0$ ), despite the fact that both systems have the same transfer function.

In Remark 16, we will show how to extract the greatest possible amount of energy from this circuit. Following that remark, we let $i=-\left(D+D^{T}\right)^{-1}\left(C-B^{T} X_{-}\right) \mathbf{x}=\frac{1}{2}\left(i_{1}+\right.$ $\left.i_{2}-v_{3}-v_{4}\right)$. From the variation of the constants formula (2),

$$
\left[\begin{array}{l}
i_{1} \\
i_{2} \\
v_{3} \\
v_{4}
\end{array}\right](t)=\frac{1}{2}\left[\begin{array}{c}
\left(\cos (t)+e^{-t}\right)\left(i_{1}-i_{2}\right)(0)+\sin (t)\left(v_{3}-v_{4}\right)(0) \\
\left(-\cos (t)+e^{-t}\right)\left(i_{1}-i_{2}\right)(0)-\sin (t)\left(v_{3}-v_{4}\right)(0) \\
-\sin (t)\left(i_{1}-i_{2}\right)(0)+\left(\cos (t)+e^{-t}\right)\left(v_{3}-v_{4}\right)(0) \\
\sin (t)\left(i_{1}-i_{2}\right)(0)+\left(-\cos (t)+e^{-t}\right)\left(v_{3}-v_{4}\right)(0)
\end{array}\right],
$$

whereupon $v(t)=-\frac{1}{2} e^{-t}\left(\left(i_{1}+i_{2}-v_{3}-v_{4}\right)(0)\right)=-i(t)$. It can then be verified that $\int_{0}^{\infty}-i(t) v(t) d t=\frac{1}{8}\left(\left(i_{1}+i_{2}-v_{3}-\right.\right.$ $\left.\left.v_{4}\right)(0)\right)^{2}=S_{a}^{\sigma_{p}}\left(\mathbf{x}_{0}\right)$.

Now, consider the circuit on the right of Fig. 11 We let

$$
A=\left[\begin{array}{cc}
0 & 1 \\
-1 & 0
\end{array}\right], B=\left[\begin{array}{l}
0 \\
2
\end{array}\right], C=\left[\begin{array}{ll}
0 & 1
\end{array}\right] \text {, and } D=0 \text {. }
$$

From Theorem 10 , we find that $S_{a}^{\sigma_{p}}(\mathbf{x}(0))=\frac{1}{4}\left(\left(i_{1}+i_{2}\right)(0)^{2}+\right.$ $\left.\left(v_{3}+v_{4}\right)(0)^{2}\right)$. Again, Remark 16 explains how to extract the greatest amount of energy from this circuit. In that remark,

$A_{\epsilon}=\left[\begin{array}{cc}0 & 1 \\ -1 & -2 \epsilon\end{array}\right], B_{\epsilon}=\left[\begin{array}{c}0 \\ 2 \sqrt{1+\epsilon^{2}}\end{array}\right], C_{\epsilon}=\left[\begin{array}{ll}0 & \frac{1-\epsilon^{2}}{\sqrt{1+\epsilon^{2}}}\end{array}\right], D_{\epsilon}=\epsilon$, $X_{-}^{\epsilon}=\frac{(1-\epsilon)^{2}}{2\left(1+\epsilon^{2}\right)} I, u_{\epsilon}=-\frac{1-\epsilon}{\sqrt{1+\epsilon^{2}}}\left(v_{3}+v_{4}\right)$, and $u=-\left(v_{3}+v_{4}\right)=-y$.

We then let $i=u$ and $v=y$. In this case, $i$ and $v$ are independent of $\epsilon$, and it can be verified that $i(t)=$ $t e^{-t}\left(i_{1}+i_{2}\right)(0)+\left(t e^{-t}-e^{-t}\right)\left(v_{3}+v_{4}\right)(0)=-v(t)$ and $\int_{0}^{\infty}-i(t) v(t) d t=\frac{1}{4}\left(\left(i_{1}+i_{2}\right)(0)^{2}+\left(v_{3}+v_{4}\right)(0)^{2}\right)=S_{a}^{\sigma_{p}}\left(\mathbf{x}_{0}\right)$.

We end this section with a remark about the optimal control problem in Definition 6 concerning the required energy.

Remark 14: The required energy $S_{r}^{\sigma_{p}}\left(\mathbf{x}_{0}\right)$ is not considered in Theorems 10, 12 and 13 If $\mathcal{B}_{s}$ is as in (1) and $(A, B)$ is controllable, then $S_{r}^{\sigma_{p}}\left(\mathbf{x}_{0}\right)$ corresponds to the energy required to transfer the state to $\mathbf{x}_{0}$ from the origin. However, if $\mathcal{B}_{s}$ is not controllable, then there exist $\mathbf{x}_{0} \in \mathbb{R}^{d}$ which cannot be reached from the origin, so the required energy for such states is undefined. Indeed, the controllability of $(A, B)$ is related to the existence of an upper bound to the set of $X \in \mathbb{R}_{s}^{d \times d}$ which satisfy condition 3 of Theorem 10 . Specifically, if there exist $\mathbf{z} \in \mathbb{C}^{d}$ and $\lambda \in \mathbb{C}$ such that $\mathbf{z}^{T}\left[\begin{array}{ll}\lambda I-A & B\end{array}\right]=0$, then $\mathbf{z}^{T} \frac{d \mathbf{x}}{d t}=\lambda \mathbf{z}^{T} \mathbf{x}$, and so $\mathbf{z}^{T} \mathbf{x}(t)=e^{\lambda\left(t-t_{0}\right)} \mathbf{z}^{T} \mathbf{x}\left(t_{0}\right)$ for all $t \in \mathbb{R}$, whence $\mathbf{z}^{T} \mathbf{x}\left(t_{0}\right) \neq 0$ implies that $\mathbf{x}(t) \neq 0$ for all $t \in \mathbb{R}$. If, in addition, $\lambda \in \overline{\mathbb{C}}_{-}$, then there are no trajectories satisfying $\mathbf{z}^{T} \mathbf{x}\left(t_{0}\right) \neq 0$ and $\lim _{t \rightarrow-\infty}(\mathbf{x}(t))=0$. 

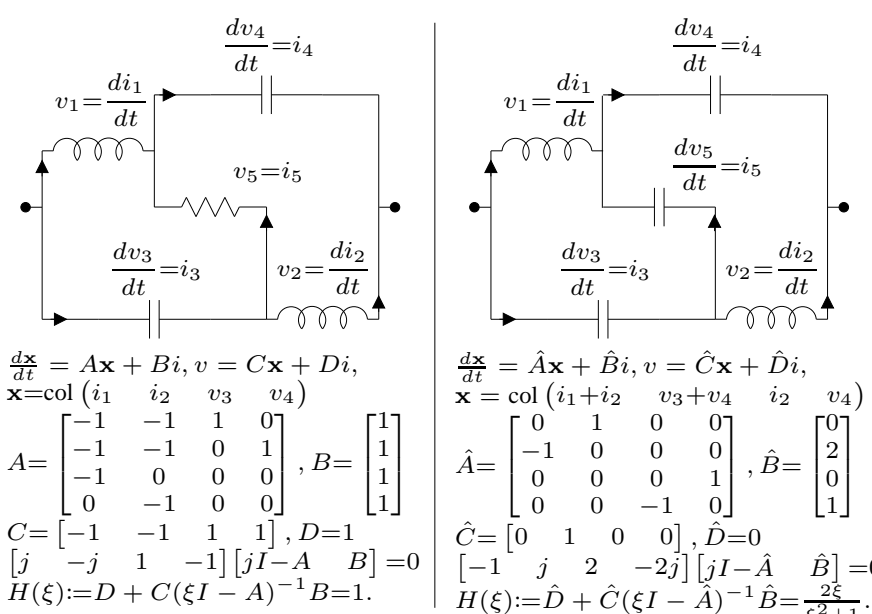

$\frac{d \mathbf{x}}{d t}=\hat{A} \mathbf{x}+\hat{B} i, v=\hat{C} \mathbf{x}+\hat{D} i$, $\mathbf{x}=\operatorname{col}\left(\begin{array}{llll}i_{1}+i_{2} & v_{3}+v_{4} & i_{2} & v_{4}\end{array}\right)$ $\hat{A}=\left[\begin{array}{cccc}0 & 1 & 0 & 0 \\ -1 & 0 & 0 & 0 \\ 0 & 0 & 0 & 1 \\ 0 & 0 & -1 & 0\end{array}\right], \hat{B}=\left[\begin{array}{l}0 \\ 2 \\ 0 \\ 1\end{array}\right]$ $\hat{C}=\left[\begin{array}{llll}0 & 1 & 0 & 0\end{array}\right], \hat{D}=0$ $\left[\begin{array}{llll}-1 & j & 2 & -2 j\end{array}\right]\left[\begin{array}{lll}j I-\hat{A} & \hat{B}\end{array}\right]=0$ $H(\xi):=\hat{D}+\hat{C}(\xi I-\hat{A})^{-1} \hat{B}=\frac{2 \xi}{\xi^{2}+1}$
Fig. 1. Two electric circuits with uncontrollable and unobservable state-space representations.

In fact, for a passive system, the following two conditions are equivalent: (i) the LMI in condition 4 of Lemma 7 has no upper bound; and (ii) there exists $0 \neq \mathbf{z} \in \mathbb{C}^{d}$ and $\lambda \in \overline{\mathbb{C}}_{-}$such that $\mathbf{z}^{T}\left[\begin{array}{ll}\lambda I-A & B\end{array}\right]=0$. To see that (ii) $\Rightarrow$ (i), let $X \in \mathbb{R}_{s}^{d \times d}$ satisfy $\Omega(X) \geq 0$ and $\hat{X}:=\mathbf{z}^{T}+\overline{\mathbf{z}} \mathbf{z}^{T}$. Then, for any given $\alpha>0,0 \leq X+\alpha \hat{X} \in \mathbb{R}_{s}^{d \times d}$ satisfies $\Omega(X+\alpha \hat{X})=\Omega(X)-\alpha(\lambda+\bar{\lambda}) \operatorname{diag}(\hat{X} \quad 0) \geq 0$. Conversely, if (ii) does not hold, then there exists $K \in \mathbb{R}^{d \times n}$ such that $\operatorname{spec}(A+B K) \in \mathbb{C}_{+}$. Then, for any given $\mathbf{x}_{0} \in \mathbb{R}^{d}$, there exists a trajectory $(\mathbf{u}, \mathbf{y}, \mathbf{x}) \in \mathcal{B}_{s}$ with $\mathbf{u}=K \mathbf{x}$, $\mathbf{x}\left(t_{0}\right)=\mathbf{x}_{0}$ and $\lim _{t \rightarrow-\infty}(\mathbf{x}(t))=0$. Finally, for this trajectory, it can be shown that (a) there exists $\hat{X} \in \mathbb{R}_{s}^{d \times d}$ such that $\int_{-\infty}^{t_{0}}\left(\mathbf{u}^{T} \mathbf{y}\right)(t) d t \leq \frac{1}{2} \mathbf{x}_{0}^{T} \hat{X} \mathbf{x}_{0}$; and (b) if $X$ satisfies condition 4 of Lemma 7, then $\frac{1}{2} \mathbf{x}_{0}^{T} X \mathbf{x}_{0} \leq \int_{-\infty}^{t_{0}}\left(\mathbf{u}^{T} \mathbf{y}\right)(t) d t$. It follows that (i) does not hold.

The following two sections provide the proofs of Theorems 10, 12 and 13. Then, in Sections VI-VIII we state and prove three analogous theorems relevant to non-expansive systems.

\section{PASSIVE SYSTEMS AND THE AVAILABLE ENERGY}

In this section, we prove Theorem 10. The proof uses the concept of storage functions and the results in Appendix B

Proof of Theorem 10 . That $2 \Longleftrightarrow 3$ is shown in [5]. Here, we prove $1 \Rightarrow 4 \Rightarrow 3 \Rightarrow 1$

$1 \Rightarrow$ 4. First, consider a $k>0$ such that $I+k D$ is nonsingular, and let $\mathbf{z} \in \mathbb{R}^{d}$ be fixed but arbitrary. Then, let

$$
\tilde{C}:=(I+k D)^{-1} C \text { and } \tilde{A}:=A-k B(I+k D)^{-1} C ;
$$

let $\tilde{\mathbf{x}}(t)=e^{\tilde{A}\left(t-t_{0}\right)} \mathbf{z}$ for all $t \in \mathbb{R}$; and let $\tilde{\mathbf{u}}=-k \tilde{C} \tilde{\mathbf{x}}$ and $\tilde{\mathbf{y}}=\tilde{C} \tilde{\mathbf{x}}$. It can be verified that $(\tilde{\mathbf{u}}, \tilde{\mathbf{y}}, \tilde{\mathbf{x}}) \in \mathcal{B}_{s}, \tilde{\mathbf{x}}\left(t_{0}\right)=\mathbf{z}$, and $\int_{t_{0}}^{t_{1}}-\left(\tilde{\mathbf{u}}^{T} \tilde{\mathbf{y}}\right)(t) d t=k \int_{t_{0}}^{t_{1}}\left(\tilde{\mathbf{y}}^{T} \tilde{\mathbf{y}}\right)(t) d t \geq 0$. Thus, $S_{a}^{\sigma_{p}}\left(\mathbf{x}_{0}\right)=$ $\frac{1}{2} \mathbf{x}_{0}^{T} X_{-} \mathbf{x}_{0}$ for some $X_{-} \in \mathbb{R}_{s}^{d \times d}$ with $X_{-} \geq 0$ by Lemma B.4 (it is conventional to include the $\frac{1}{2}$ ). It remains to show that $X_{-}$satisfies conditions 4 (ii)-(iv).

The proof of 4 (iii) is inspired by [2, Proof of Lemma 1]. Note initially that, since $X_{-} \geq 0$, then any given $\mathbf{z} \in \mathbb{R}^{d}$ satisfies $X_{-} \mathbf{z}=0 \Longleftrightarrow \mathbf{z}^{T} X_{-} \mathbf{z}=0 \Longleftrightarrow S_{a}^{\sigma_{p}}(\mathbf{z})=0$, whence $V_{o} \mathbf{z}=0 \Rightarrow X_{-} \mathbf{z}=0$ by Lemma B.5. Now, let $\tilde{\mathbf{u}}, \tilde{\mathbf{y}}$, and $\tilde{\mathbf{x}}$ be as in the previous paragraph, so $\int_{t_{0}}^{t_{1}}-\left(\tilde{\mathbf{u}}^{T} \tilde{\mathbf{y}}\right)(t) d t=$ $k \int_{t_{0}}^{t_{1}}\left(\tilde{\mathbf{y}}^{T} \tilde{\mathbf{y}}\right)(t) d t \leq S_{a}^{\sigma_{p}}(\mathbf{z})$. If $X_{-} \mathbf{z}=0$, then $0=S_{a}^{\sigma_{p}}(\mathbf{z}) \geq$ $k \int_{t_{0}}^{t_{1}}\left(\tilde{\mathbf{y}}^{T} \tilde{\mathbf{y}}\right)(t) d t$, so $\tilde{\mathbf{y}}(t)=\tilde{C} e^{\tilde{A}\left(t-t_{0}\right)} \mathbf{z}=0$ for all $t \geq t_{0}$. This implies $\tilde{C} \tilde{A}^{k} \mathbf{z}=0(k=0,1,2, \ldots)$, which implies $C A^{k} \mathbf{z}=0(k=0,1,2, \ldots)$, hence $V_{o} \mathbf{z}=0$.

To prove 4 (ii), note from Lemma B.3 that $S_{a}^{\sigma_{p}}$ is a storage function (with respect to $\mathbf{u}^{T} \mathbf{y}$ ). Thus, $S_{a}^{\sigma_{p}}\left(\mathbf{x}\left(t_{1}\right)\right) \leq$ $\int_{t_{0}}^{t_{1}}\left(\mathbf{u}^{T} \mathbf{y}\right)(t) d t+S_{a}^{\sigma_{p}}\left(\mathbf{x}\left(t_{0}\right)\right)$ for all $(\mathbf{u}, \mathbf{y}, \mathbf{x}) \in \mathcal{B}_{s}$ and $t_{1} \geq t_{0}$. From the variation of the constants formula (2) (3), for any given $\mathbf{x}_{0} \in \mathbb{R}^{d}$ and $\mathbf{u}_{0} \in \mathbb{R}^{n}$, there exists $\mathrm{a}(\mathbf{u}, \mathbf{y}, \mathbf{x}) \in \mathcal{B}_{s}$ with $\mathbf{x}$ differentiable, $\mathbf{x}\left(t_{0}\right)=\mathbf{x}_{0}$, and $\mathbf{u}\left(t_{0}\right)=\mathbf{u}_{0}$. Thus, $\left(\mathbf{u}^{T} \mathbf{y}\right)\left(t_{0}\right)-\frac{d}{d t}\left(S_{a}^{\sigma_{p}}(\mathbf{x})\right)\left(t_{0}\right)=$ $\frac{1}{2}\left[\begin{array}{ll}\mathbf{x}_{0}^{T} & \mathbf{u}_{0}^{T}\end{array}\right] \Omega\left(X_{-}\right) \operatorname{col}\left(\mathbf{x}_{0} \quad \mathbf{u}_{0}\right) \geq 0$, and so $\Omega\left(X_{-}\right) \geq 0$.

To prove 4 (iv), note that if $X \geq 0$ and $\Omega(X) \geq 0$, then

$$
\begin{aligned}
& 2 \int_{t_{0}}^{t_{1}}\left(\mathbf{u}^{T} \mathbf{y}\right)(t) d t-\left[\left(\mathbf{x}^{T} X \mathbf{x}\right)(t)\right]_{t_{0}}^{t_{1}} \\
&=\int_{t_{0}}^{t_{1}}\left(\left[\begin{array}{ll}
\mathbf{x}^{T} & \mathbf{u}^{T}
\end{array}\right] \Omega(X) \operatorname{col}\left(\begin{array}{ll}
\mathbf{x} & \mathbf{u}
\end{array}\right)\right)(t) d t
\end{aligned}
$$

so $\frac{1}{2} \mathbf{x}\left(t_{1}\right)^{T} X \mathbf{x}\left(t_{1}\right) \leq \int_{t_{0}}^{t_{1}}\left(\mathbf{u}^{T} \mathbf{y}\right)(t) d t+\frac{1}{2} \mathbf{x}\left(t_{0}\right)^{T} X \mathbf{x}\left(t_{0}\right)$. With $S\left(\mathbf{x}_{0}\right):=\frac{1}{2} \mathbf{x}_{0}^{T} X \mathbf{x}_{0}$ for all $\mathbf{x}_{0} \in \mathbb{R}^{d}$, it follows that $S$ is a storage function. Thus, $\mathbf{x}_{0}^{T} X \mathbf{x}_{0} \geq \mathbf{x}_{0}^{T} X_{-} \mathbf{x}_{0}$ for all $\mathbf{x}_{0} \in \mathbb{R}^{d}$ by Lemma B.3, which implies $X \geq X_{-}$.

\section{$4 \Rightarrow$ Immediate.}

$3 \Rightarrow$ 1. Recall from the proof of $1 \Rightarrow 4$ iv) that $S\left(\mathbf{x}_{0}\right)=\frac{1}{2} \mathbf{x}_{0}^{T} X \mathbf{x}_{0}$ is a storage function (with respect to $\mathbf{u}^{T} \mathbf{y}$ ). Condition 1 then follows from Lemma B.3.

It remains to show that, if $(C, A)$ is observable and conditions 14 hold, then (i) $\operatorname{spec}(A) \in \overline{\mathbb{C}}_{-}$; and (ii) if $X \in \mathbb{R}_{s}^{d \times d}$ satisfies $\Omega(X) \geq 0$, then $X_{-} \leq X$.

Condition (i) follows from [3], Theorem 3.7.5], as condition 4 implies that $X_{-}>0$ and $-A^{T} X_{-}-X_{-} A \geq 0$.

To see (ii), let $X \in \mathbb{R}_{s}^{d \times d}$ satisfy $\Omega(X) \geq 0$, and note that (12) holds. Then, for any given $\mathbf{x}_{0} \in \mathbb{R}^{d}$ and $\epsilon>0$, there exists $(\mathbf{u}, \mathbf{y}, \mathbf{x}) \in \mathcal{B}_{s}$ with $\mathbf{x}\left(t_{0}\right)=\mathbf{x}_{0}$ and $t_{1} \geq t_{0}$ such that

$\int_{t_{0}}^{t_{1}}-\left(\mathbf{u}^{T} \mathbf{y}\right)(t) d t=S_{a}^{\sigma_{p}}\left(\mathbf{x}_{0}\right)-\epsilon \leq \frac{1}{2}\left(\mathbf{x}_{0}^{T} X \mathbf{x}_{0}-\mathbf{x}\left(t_{1}\right)^{T} X \mathbf{x}\left(t_{1}\right)\right)$.

We will show that there exists $M \in \mathbb{R}$ (independent of $\epsilon$ ) such that $\mathbf{x}\left(t_{1}\right)^{T} X \mathbf{x}\left(t_{1}\right) \geq M \epsilon$. This implies that $\mathbf{x}_{0}^{T} X_{-} \mathbf{x}_{0} \leq$ $\mathbf{x}_{0}^{T} X \mathbf{x}_{0}+\epsilon(1-M)$. Since $\epsilon$ can be chosen to be arbitrarily small, we conclude that $X_{-} \leq X$

To obtain the bound $M$, let $k>0$ be such that $I+k D$ is nonsingular; let $\tilde{C}$ and $\tilde{A}$ be as in 111 ; let $\mathbf{x}\left(t_{1}\right)=\mathbf{x}_{1}$; and let $(\tilde{\mathbf{u}}(t), \tilde{\mathbf{y}}(t), \tilde{\mathbf{x}}(t))=(\mathbf{u}(t), \mathbf{y}(t), \mathbf{x}(t))$ for all $t_{0} \leq t<t_{1}$, and $\tilde{\mathbf{x}}(t)=e^{\tilde{A}\left(t-t_{1}\right)} \mathbf{x}_{1}, \tilde{\mathbf{u}}(t)=-k \tilde{C} \mathbf{x}(t)$, and $\tilde{\mathbf{y}}(t)=\tilde{C} \mathbf{x}(t)$ for all $t \geq t_{1}$. Then $(\tilde{\mathbf{u}}, \tilde{\mathbf{y}}, \tilde{\mathbf{x}}) \in \mathcal{B}_{s}$ with $\tilde{\mathbf{x}}\left(t_{0}\right)=\mathbf{x}_{0}$. Next, consider a fixed $T>0$, and let $\tilde{\mathcal{O}}:=\int_{0}^{T} e^{\tilde{A}^{T} \tau} \tilde{C}^{T} \tilde{C} e^{\tilde{A} \tau} d \tau$. From earlier in the proof, $(\tilde{C}, \tilde{A})$ is observable since $(C, A)$ is, and so $\tilde{\mathcal{O}}>0$. Moreover,

$\int_{t_{0}}^{t_{1}+T}-\left(\tilde{\mathbf{u}}^{T} \tilde{\mathbf{y}}\right)(t) d t=S_{a}^{\sigma_{p}}\left(\mathbf{x}_{0}\right)-\epsilon+k \mathbf{x}_{1}^{T} \tilde{\mathcal{O}} \mathbf{x}_{1} \leq S_{a}^{\sigma_{p}}\left(\mathbf{x}_{0}\right)$,

so $\mathbf{x}_{1}^{T} \tilde{\mathcal{O}} \mathbf{x}_{1} \leq \epsilon / k$. Now, let $\lambda>0$ denote the least eigenvalue of $\tilde{\mathcal{O}}$. Also, if $X \geq 0$ we let $\mu:=0$, and otherwise we let $\mu<0$ be the most negative eigenvalue of $X$. By Rayleigh's quotient, $\mathbf{x}_{1}^{T} X \mathbf{x}_{1} \geq \mu \mathbf{x}_{1}^{T} \mathbf{x}_{1} \geq \frac{\mu}{\lambda} \mathbf{x}_{1}^{T} \tilde{\mathcal{O}} \mathbf{x}_{1} \geq \frac{\mu \epsilon}{k \lambda}$, which gives the bound $M:=\mu /(k \lambda)$. 


\section{EXPLICIT CHARACTERISATION OF THE AVAILABLE ENERGY}

In this section, we prove Theorems 12 and 13 . We also show how to compute the available energy of a passive system.

Proof of Theorem 12. With the notation

$$
S(X):=\left[\begin{array}{cc}
I & 0 \\
\left(D+D^{T}\right)^{-1}\left(B^{T} X-C\right) & I
\end{array}\right],
$$

then $S(X)$ is nonsingular and

$$
S(X)^{T} \Omega(X) S(X)=\left[\begin{array}{cc}
\Gamma(X) & 0 \\
0 & D+D^{T}
\end{array}\right] .
$$

Thus, $\Omega(X) \geq 0 \Longleftrightarrow \Gamma(X) \geq 0$.

$2 \Rightarrow$ 1, From (14), $X_{-} \geq 0$ and $\Omega\left(X_{-}\right) \geq 0$, so $S_{a}^{\sigma_{p}}\left(\mathbf{x}_{0}\right)<\infty$ for all $\mathbf{x}_{0} \in \mathbb{R}^{d}$ by Theorem 10 .

$1 \Rightarrow 2 \quad$ Since $S_{a}^{\sigma_{p}}\left(\mathrm{x}_{0}\right)<\infty$ for all $\mathrm{x}_{0} \in \mathbb{R}^{d}$, then $S_{a}^{\sigma_{p}}\left(\mathbf{x}_{0}\right)=\frac{1}{2} \mathbf{x}_{0}^{T} X_{-} \mathbf{x}_{0}$ for some $X_{-} \in \mathbb{R}_{s}^{d \times d}$ satisfying (i) $X_{-} \geq 0$, (ii) $\Omega\left(X_{-}\right) \geq 0$, and (iii) if $\mathbf{z} \in \mathbb{R}^{d}$, then $X_{-} \mathbf{z}=$ $0 \Longleftrightarrow V_{o} \mathbf{z}=0$, by Theorem 10 . It remains to show that conditions 2(ii) and 2(iv) are also satisfied.

To show condition 2(ii), we let $\sigma(\mathbf{u}, \mathbf{y})=\mathbf{u}^{T} \mathbf{y}$. From the proof of Theorem 10, $\sigma$ satisfies the conditions of Lemma B.4 so (35) holds from the proof of that lemma. Also, for any given $t_{2} \geq t_{1} \geq t_{0}$ and $(\mathbf{u}, \mathbf{y}, \mathbf{x}) \in \mathcal{B}_{s}$ with $\mathbf{x}\left(t_{0}\right)=\mathbf{x}_{0}$,

$$
\begin{aligned}
\int_{t_{0}}^{t_{2}} & -\sigma(\mathbf{u}, \mathbf{y})(t) d t \leq \int_{t_{0}}^{t_{1}}-\sigma(\mathbf{u}, \mathbf{y})(t) d t+S_{a}^{\sigma_{p}}\left(\mathbf{x}\left(t_{1}\right)\right) \\
& =S_{a}^{\sigma_{p}}\left(\mathbf{x}_{0}\right)-\frac{1}{2} \int_{t_{0}}^{t_{1}}\left(\left[\begin{array}{ll}
\mathbf{x}^{T} & \mathbf{u}^{T}
\end{array}\right] \Omega\left(X_{-}\right) \operatorname{col}\left(\begin{array}{ll}
\mathbf{x} & \mathbf{u}
\end{array}\right)\right)(t) d t .
\end{aligned}
$$

By taking the supremum over all $t_{2} \geq t_{1}$ and $\mathbf{u} \in \mathcal{L}_{2}^{\text {loc }}\left(\mathbb{R}, \mathbb{R}^{n}\right)$, and using (35) from the proof of Lemma B.4 we find that

$$
\begin{array}{r}
0 \leq \sup _{\mathbf{u} \in \mathcal{L}_{2}^{\text {loc }}\left(\mathbb{R}, \mathbb{R}^{n}\right)} \int_{t_{0}}^{t_{1}}-\left(\left[\begin{array}{ll}
\mathbf{x}^{T} & \mathbf{u}^{T}
\end{array}\right] \Omega\left(X_{-}\right) \operatorname{col}\left(\begin{array}{ll}
\mathbf{x} & \mathbf{u}
\end{array}\right)\right)(t) d t \\
\text { such that }(\mathbf{u}, \mathbf{y}, \mathbf{x}) \in \mathcal{B}_{s}, \mathbf{x}\left(t_{0}\right)=\mathbf{x}_{0}, \quad(15)
\end{array}
$$

for any given $\mathbf{x}_{0} \in \mathbb{R}^{d}$ and $t_{1} \geq t_{0} \in \mathbb{R}$. Since $\Omega\left(X_{-}\right) \geq 0$, then the above inequality must be satisfied with equality. We let $\mathbf{v}:=\mathbf{u}+\left(D+D^{T}\right)^{-1}\left(C-B^{T} X_{-}\right) \mathbf{x}$, so (13)-15) imply

$$
\begin{aligned}
& 0=\inf _{\mathbf{v} \in \mathcal{L}_{2}^{\text {loc }}\left(\mathbb{R}, \mathbb{R}^{n}\right)} \int_{t_{0}}^{t_{1}}\left(\mathbf{x}^{T} \Gamma\left(X_{-}\right) \mathbf{x}+\mathbf{v}^{T}\left(D+D^{T}\right) \mathbf{v}\right)(t) d t \\
& \text { such that } \mathbf{x} \in \mathcal{L}_{2}^{\text {loc }}\left(\mathbb{R}, \mathbb{R}^{d}\right), \frac{d \mathbf{x}}{d t}=A_{\Gamma}\left(X_{-}\right) \mathbf{x}+B \mathbf{v}, \mathbf{x}\left(t_{0}\right)=\mathbf{x}_{0} .
\end{aligned}
$$

From [18, Section 2.3], for any given $t_{1} \geq t_{0}$, the above infimum is equal to $\mathbf{x}_{0}^{T} P\left(t_{0}-t_{1}\right) \mathbf{x}$, where $P$ is an absolutely continuous matrix function that satisfies $P(0)=0$ and

$$
\begin{aligned}
-\frac{d P}{d t}=P A_{\Gamma}( & \left.X_{-}\right)+A_{\Gamma}\left(X_{-}\right)^{T} P \\
& -P B\left(D+D^{T}\right)^{-1} B^{T} P+\Gamma\left(X_{-}\right) .
\end{aligned}
$$

Since $\mathbf{x}_{0} \in \mathbb{R}^{d}$ can be chosen arbitrarily, then $P(t)=\frac{d P}{d t}(t)=$ 0 for all $t<0$, and so $\Gamma\left(X_{-}\right)=0$ by (16).

To show condition 2 (iv), we consider the cases: (i) $(C, A)$ observable; and (ii) $(C, A)$ not observable.

Case (i): $(C, A)$ observable. We note that $\left[\begin{array}{ll}\lambda I-A & B\end{array}\right]$ has full row rank for all $\lambda \in \overline{\mathbb{C}}_{+}$(see Remarks 4 and 9 ). This implies that $\left[\lambda I-A_{\Gamma}\left(X_{-}\right) \quad B\right]$ has full row rank for all $\lambda \in \overline{\mathbb{C}}_{+}$, so $\left(A_{\Gamma}\left(X_{-}\right), B\right)$ is stabilizable. The proof of this condition is then identical to [4, Lemma 7].
Case (ii): $(C, A)$ not observable. Consider the observer staircase form (see note $\mathrm{A}\left[1\right.$, and let $\mathcal{B}_{s}$ and $\hat{S}_{a}^{\sigma}$ be as in Lemma B.5 (with $\sigma(\mathbf{u}, \mathbf{y})=\mathbf{u}^{T} \mathbf{y}$ ). It follows from Lemma B.5 that $X_{-}=T^{T} \operatorname{diag}\left(\hat{X}_{-} \quad 0\right) T$ where $\hat{X}_{-} \in \mathbb{R}_{s}^{\hat{d} \times \hat{d}}$ with $\frac{1}{2} \hat{\mathbf{x}}_{0}^{T} \hat{X}_{-} \hat{\mathbf{X}}_{0}=\hat{S}_{a}^{\sigma_{p}}\left(\hat{\mathbf{x}}_{0}\right)$ for all $\hat{\mathbf{x}}_{0} \in \mathbb{R}^{\hat{d}}$. With

$\hat{\Gamma}(\hat{X}):=-A_{11}^{T} \hat{X}-\hat{X} A_{11}-\left(C_{1}^{T}-\hat{X} B_{1}\right)\left(D+D^{T}\right)^{-1}\left(C_{1}-B_{1}^{T} \hat{X}\right)$, and $A_{\hat{\Gamma}}(\hat{X}):=A_{11}-B_{1}\left(D+D^{T}\right)^{-1}\left(C_{1}-B_{1}^{T} \hat{X}\right)$,

it follows from case (i) that $\hat{X}_{-} \geq 0, \hat{\Gamma}\left(\hat{X}_{-}\right)=0$, and $\operatorname{spec}\left(A_{\hat{\Gamma}}\left(\hat{X}_{-}\right)\right) \in \overline{\mathbb{C}}_{-}$. Also, it can be verified that $\Gamma\left(X_{-}\right)=$ $T^{T} \operatorname{diag}\left(\hat{\Gamma}\left(\hat{X}_{-}\right) \quad 0\right) T$; and

$$
T A_{\Gamma}\left(X_{-}\right) T^{-1}=\left[\begin{array}{cc}
A_{\hat{\Gamma}^{(}}\left(\hat{X}_{-}\right) & 0 \\
\hat{A}_{21} & A_{22}
\end{array}\right],
$$

where $\hat{A}_{21}:=A_{21}-B_{2}\left(D+D^{T}\right)^{-1}\left(C_{1}^{T}-B_{1}^{T} \hat{X}_{-}\right)$. Now, suppose $\lambda \in \mathbb{C}_{+}$and $\mathbf{z} \in \mathbb{C}^{d}$ satisfy $A_{\Gamma}\left(X_{-}\right) \mathbf{z}=\lambda \mathbf{z}$, and let $T_{1}$ be as in note $\mathrm{A}$.1. Since $\lambda I-A_{\hat{\Gamma}}\left(\hat{X}_{-}\right)$is nonsingular for all $\lambda \in \mathbb{C}_{+}$, then (18) implies that $T_{1} \mathbf{z}=0$, and it is then easily shown that $V_{o} \mathbf{z}=0$.

It remains to show that if $X_{-}$satisfies condition 2, then $S_{a}^{\sigma_{p}}\left(\mathbf{x}_{0}\right)=\frac{1}{2} \mathbf{x}_{0}^{T} X_{-} \mathbf{x}_{0}$. To prove this, we assume that $(C, A)$ is observable, and we show that if $X \in \mathbb{R}_{s}^{d \times d}$ satisfies $X \geq 0$, $X \neq X_{-}$, and $\Gamma(X)=0$, then $\operatorname{spec}\left(A_{\Gamma}(X)\right) \notin \overline{\mathbb{C}}_{-}$. The case with $(C, A)$ not observable can then be shown by considering the observer staircase form as in the proof of case (ii) above.

If $\Gamma(X)=0$, then $\Omega(X) \geq 0$, so $Y:=X-$ $X_{-} \geq 0$ by Theorem 10. Also, by direct calculation, $A_{\Gamma}\left(X_{-}\right)^{T} Y+Y A_{\Gamma}\left(X_{-}\right)+Y B\left(D+D^{T}\right)^{-1} B^{T} Y=0$. From before, $\left(A_{\Gamma}\left(X_{-}\right), B\right)$ is stabilizable, so from [4, Proof of Lemma A.1] we find that $A_{\Gamma}\left(X_{-}\right)+B\left(D+D^{T}\right)^{-1} B^{T} Y=$ $A_{\Gamma}(X)$ satisfies $\operatorname{spec}\left(A_{\Gamma}(X)\right) \notin \overline{\mathbb{C}}_{-}$.

Remark 15: From the proof of Theorems 10 and 12 in order to find the matrix $X_{-}$in those theorems, it suffices to find an $X_{-} \in \mathbb{R}_{s}^{d \times d}$ satisfying $\Gamma\left(X_{-}\right)=0$ and $\operatorname{spec}\left(A_{\Gamma}\left(X_{-}\right)\right) \in \overline{\mathbb{C}}_{-}$ for the case with $(C, A)$ observable. This can be obtained from the controller staircase form [3, Theorem 3.3.4]:

$T A T^{-1}=\left[\begin{array}{cc}A_{11} & A_{12} \\ 0 & A_{22}\end{array}\right], T B=\left[\begin{array}{c}B_{1} \\ 0\end{array}\right], C T^{-1}=\left[\begin{array}{ll}C_{1} & C_{2}\end{array}\right]$,

with $\left(A_{11}, B_{1}\right)$ controllable. Since $(C, A)$ is observable, then so too is $\left(C_{1}, A_{11}\right)$. Furthermore, $D+C_{1}\left(\xi I-A_{11}\right)^{-1} B_{1}=$ $D+C(\xi I-A)^{-1} B$, which is positive-real (see Remark 9). Thus, with $\hat{\Gamma}$ and $A_{\hat{\Gamma}}$ as in (17), there exists a unique $X_{11}>0$ satisfying $\hat{\Gamma}\left(X_{11}\right)=0$ and $\operatorname{spec}\left(A_{\hat{\Gamma}}\left(X_{11}\right)\right) \in \overline{\mathbb{C}}_{-}$[2], Lemma 2]. This can be efficiently computed using the methods in [3, Chapter 6]. Next, note that $(A, B)$ is stabilizable since $(C, A)$ is observable (see Remarks 4 and 9], so $\operatorname{spec}\left(A_{22}\right) \in \mathbb{C}_{-}$[7], Corollary 5.2.31]. Thus, from [3, Theorem 3.7.4], there exists a unique real $X_{12}^{T}$ satisfying the Sylvester equation:

$$
\begin{aligned}
A_{22}^{T} X_{12}^{T}+ & X_{12}^{T} A_{\hat{\Gamma}}\left(X_{11}\right) \\
& =-A_{12}^{T} X_{11}-C_{2}^{T}\left(D+D^{T}\right)^{-1}\left(C_{1}-B_{1}^{T} X_{11}\right)
\end{aligned}
$$

and a unique real $Z \geq 0$ satisfying the Lyapunov equation:

$$
\begin{aligned}
& -\left(A_{22}^{T} Z+Z A_{22}\right) \\
& \quad=\left(C_{2}^{T}-X_{12}^{T} X_{11}^{-1} C_{1}^{T}\right)\left(D+D^{T}\right)^{-1}\left(C_{2}-C_{1} X_{11}^{-1} X_{12}\right)
\end{aligned}
$$


Then, with the notation

$$
\begin{aligned}
\hat{A}_{12} & :=A_{12}-B_{1}\left(D+D^{T}\right)^{-1}\left(C_{2}-B_{1}^{T} X_{12}\right), \\
\text { and } X_{-} & =T^{T}\left[\begin{array}{cc}
X_{11} & X_{12} \\
X_{12}^{T} & Z+X_{12}^{T} X_{11}^{-1} X_{12}
\end{array}\right] T,
\end{aligned}
$$

it can be verified that $X_{-} \geq 0, \Gamma\left(X_{-}\right)=0$, and

$$
T A_{\Gamma}\left(X_{-}\right) T^{-1}=\left[\begin{array}{cc}
A_{\hat{\Gamma}}\left(X_{11}\right) & \hat{A}_{12} \\
0 & A_{22}
\end{array}\right] .
$$

This implies that $\operatorname{spec}\left(A_{\Gamma}\left(X_{-}\right)\right)=\operatorname{spec}\left(A_{\hat{\Gamma}}\left(X_{11}\right)\right) \cup$ $\operatorname{spec}\left(A_{22}\right) \in \overline{\mathbb{C}}_{-}$, so $X_{-}$is the matrix in Theorem 12

To finish this section we prove Theorem 13

Proof of Theorem 13 . That $2 \Rightarrow 1$ is immediate from Theorem 10 , since $X_{-} \geq 0$ satisfies $\Omega\left(X_{-}\right) \geq 0$. It remains to show that $1 \Rightarrow 2$ and if $X_{-}$has the properties indicated in condition 2 then $S_{a}^{\sigma_{p}}\left(\mathbf{x}_{0}\right)=\frac{1}{2} \mathbf{x}_{0}^{T} X_{-} \mathbf{x}_{0}$. We will prove this for the cases: (i) $(C, A)$ observable and $D+D^{T}>0$; (ii) $(C, A)$ observable; and finally (iii) $(C, A)$ not observable.

Case (i) $(C, A)$ observable and $D+D^{T}>0$. It suffices to show that $X_{-}$satisfies condition 2 in Theorem 12 if and only if $X_{-}$satisfies condition 2 in the present theorem.

First, let $X_{-}$satisfy condition 2 in Theorem 12 Since $D+D^{T}>0$, then there exists a nonsingular $W$ satisfying $W^{T} W=D+D^{T}$. We let $L:=\left(W^{T}\right)^{-1}\left(C-B^{T} X_{-}\right)$, and we obtain $-A^{T} X_{-}-X_{-}^{T} A-L^{T} L=\Gamma\left(X_{-}\right)=0$.

Now, let $Z\left(\underline{\xi)}:=W+L(\xi I-A)^{-1} B\right.$. From Theorems 10 and 12. $\operatorname{spec}(A) \in \overline{\mathbb{C}}_{-}$and $\operatorname{spec}\left(A_{\Gamma}\left(X_{-}\right)\right) \in \overline{\mathbb{C}}_{-}$. Also,

$$
\left[\begin{array}{cc}
\lambda I-A & -B \\
L & W
\end{array}\right]=\left[\begin{array}{cc}
\lambda I-A_{\Gamma}\left(X_{-}\right) & -B \\
0 & W
\end{array}\right]\left[\begin{array}{cc}
I & 0 \\
W^{-1} L & I
\end{array}\right] .
$$

The matrices in 20 have full row rank for all $\lambda \in \mathbb{C}_{+}$, so $Z$ is a spectral factor for $H+H^{\star}$ by Lemma D.1.

Next, let $X_{-}$satisfy condition 2 in the present theorem. Since $W+L(\xi I-A)^{-1} B$ is a spectral factor of $H+H^{\star}$, then $W$ is nonsingular. Thus, $L=\left(W^{T}\right)^{-1}\left(C-B^{T} X_{-}\right)$, and $\Gamma\left(X_{-}\right)=-A^{T} X_{-}-X_{-} A-L^{T} L=0$. As before, $\operatorname{spec}(A) \in$ $\overline{\mathbb{C}}_{-}$, so the matrices in (20) have full row rank for all $\lambda \in \mathbb{C}_{+}$ by Lemma D.1 and so $\operatorname{spec}\left(A_{\Gamma}\left(X_{-}\right)\right) \in \overline{\mathbb{C}}_{-}$.

Case (ii) $(\boldsymbol{C}, \boldsymbol{A})$ observable. Let $P$ and $Q$ be as in Theorem 10, and let $P_{1}:=P$ and $Q_{1}:=Q$. If $P_{1}$ and $Q_{1}$ do not satisfy the conditions of case (i), then we will construct $P_{m}, Q_{m} \in \mathbb{R}^{n_{m} \times n_{m}}[\xi]$ that do. Specifically, we consider the following four statements:

(R1) $P_{i}, Q_{i} \in \mathbb{R}^{n_{i} \times n_{i}}[\xi]$ where $\left(P_{i}, Q_{i}\right)$ is a positive-real pair and $Q_{i}^{-1} P_{i}$ is proper.

(R2) $D_{i}:=\lim _{\xi \rightarrow \infty}\left(Q_{i}^{-1} P_{i}(\xi)\right)$ is symmetric.

(R3) $P_{i}$ is nonsingular and $D_{i}=\operatorname{diag}\left(\begin{array}{ll}I_{r_{i}} & 0\end{array}\right)$.

(R4) $D_{i}=I$ or $n_{i}=0$.

By Theorem 10, $P_{1}$ and $Q_{1}$ satisfy condition (R1). Then, using Lemmas D.2 D.4 we construct $P_{2}, \ldots, P_{m}$, $Q_{2}, \ldots, Q_{m}$ such that condition $(\mathrm{R} 1)$ is satisfied, $n_{i} \leq n_{i-1}$, and $\operatorname{deg}\left(\operatorname{det}\left(Q_{i}\right)\right) \leq \operatorname{deg}\left(\operatorname{det}\left(Q_{i-1}\right)\right)$, for $i=2, \ldots, m$; and

1) If, for $i=k-1, \overline{(\mathrm{R} 2)}$ is not satisfied, then (R2) is satisfied for $i=k$ (Lemma D.2).

2) If, for $i=k-1,(\mathrm{R} 2)$ is satisfied but (R3) is not, then (R2) and (R3) are satisfied for $i=k$; and if $P_{k-1}$ is singular then $n_{k}<n_{k-1}$ (Lemma D.3).
3) If, for $i=k-1,(\mathrm{R} 2)$ and (R3) are satisfied but (R4) is not, then $\operatorname{deg}\left(\operatorname{det}\left(Q_{k}\right)\right)<\operatorname{deg}\left(\operatorname{det}\left(Q_{k-1}\right)\right)$ (Lemma D.4).

This inductive procedure terminates in a finite number of steps with matrices $P_{m}$ and $Q_{m}$ that satisfy conditions (R1) (R4). The procedure is inspired by the sequence of transformations outlined in [3, Section 8.4]. In contrast to [3], we also consider the case of uncontrollable systems.

Next, we consider the following four statements:

(S1) There exist polynomial matrices $M_{i}, N_{i}, U_{i}, V_{i}, E_{i}, F_{i}$ such that $\left[\begin{array}{cc}M_{i} & N_{i} \\ U_{i} & V_{i}\end{array}\right]\left[\begin{array}{ccc}-D_{i} & I & -C_{i} \\ -B_{i} & 0 & \mathcal{A}_{i}\end{array}\right]=\left[\begin{array}{ccc}-P_{i} & Q_{i} & 0 \\ -E_{i} & -F_{i} & I\end{array}\right]$, where $\mathcal{A}_{i}(\xi):=\xi I-A_{i}$, and the leftmost matrix is unimodular.

(S2) With $\Omega_{i}(X):=\left[\begin{array}{cc}-A_{i}^{T} X-X A_{i} & C_{i}^{T}-X B_{i} \\ C_{i}-B_{i}^{T} X & D_{i}+D_{i}^{T}\end{array}\right]$, then $X_{i}$ is a real matrix that satisfies (i) $X_{i} \geq 0$; (ii) $\Omega_{i}\left(X_{i}\right) \geq 0$; and (iii) if $X$ is a real matrix that satisfies $X \geq 0$ and $\Omega_{i}(X) \geq 0$, then $X_{i} \leq X$.

(S3) $X_{i}, L_{i}$ and $W_{i}$ are real matrices such that $X_{i} \geq 0$ and $\Omega_{i}\left(X_{i}\right)=\left[\begin{array}{cc}-A_{i}^{T} X_{i}-X_{i} A_{i} & C_{i}^{T}-X_{i} B_{i} \\ C_{i}-B_{i}^{T} X_{i} & D_{i}+D_{i}^{T}\end{array}\right]=\left[\begin{array}{c}L_{i}^{T} \\ W_{i}^{T}\end{array}\right]\left[\begin{array}{ll}L_{i} & W_{i}\end{array}\right]$.

(S4) $\left[\begin{array}{cc}\lambda I-A_{i} & -B_{i} \\ L_{i} & W_{i}\end{array}\right]$ has full row rank for all $\lambda \in \mathbb{C}_{+}$.

From notes $\mathrm{A}[1, \mathrm{~A} \cdot 2$ there exist real matrices $A_{m}, B_{m}, C_{m}, D_{m}$ such that condition (S1) holds. Then, from case (i), there is a unique $X_{m}$ for which there exist $L_{m}$ and $W_{m}$ that satisfy conditions (S3) and (S4). Furthermore, by Theorem 10 , this $X_{m}$ also satisfies condition (S2) Then, using Lemmas D.2 D.4 we find that there are unique $X_{i}$ for which there exist $L_{i}$ and $W_{i}$ that satisfy conditions (S3) and (S4), and these $X_{i}$ also satisfy condition (S2) $(i=m-1, \ldots, 1)$. Now, let

$$
\begin{gathered}
\hat{\mathcal{B}}_{s}:=\left\{\left(\mathbf{u}, \mathbf{y}, \mathbf{x}_{1}\right) \in \mathcal{L}_{2}^{\text {loc }}\left(\mathbb{R}, \mathbb{R}^{n}\right) \times \mathcal{L}_{2}^{\text {loc }}\left(\mathbb{R}, \mathbb{R}^{n}\right) \times \mathcal{L}_{2}^{\text {loc }}\left(\mathbb{R}, \mathbb{R}^{d_{1}}\right) \mid\right. \\
\left.\frac{d \mathbf{x}_{1}}{d t}=A_{1} \mathbf{x}_{1}+B_{1} \mathbf{u} \text { and } \mathbf{y}=C_{1} \mathbf{x}_{1}+D_{1} \mathbf{u}\right\} .
\end{gathered}
$$

Since $P=P_{1}$ and $Q=Q_{1}$, then from note $\mathrm{A} / 2$ we conclude that $\left(C_{1}, A_{1}\right)$ is observable and $\hat{\mathcal{B}}_{s}^{(\mathbf{u}, \mathbf{y})}=\mathcal{B}_{s}^{(\mathbf{u}, \mathbf{y})}$. Thus, from note $\mathrm{A}$.3. there exists a nonsingular $T \in \mathbb{R}^{d \times d}$ such that (34) holds. It can then be verified that $X_{-}:=T^{T} X_{1} T$, $L:=L_{1} T$, and $W:=W_{1}$ satisfy condition 2 in the present theorem statement; and $X_{-}$satisfies (a) $\Omega\left(X_{-}\right) \geq 0$; and (b) if $X \in \mathbb{R}_{s}^{d \times d}$ satisfies $X \geq 0$ and $\Omega(X) \geq 0$, then $X_{-} \leq X$. Since $X_{-}$is uniquely determined by conditions (a)-(b), then $S_{a}^{\sigma_{p}}\left(\mathbf{x}_{0}\right)=\frac{1}{2} \mathbf{x}_{0}^{T} X_{-} \mathbf{x}_{0}$ by Theorem 10 .

Case (iii) $(\boldsymbol{C}, \boldsymbol{A})$ not observable. Consider the observer staircase form (see note A[1), so $D+C_{1}\left(\xi I-A_{11}\right)^{-1} B_{1}=$ $D+C(\xi I-A)^{-1} B$, and let $T$ be as in note $\mathrm{A}\left[1\right.$ and $\hat{\mathcal{B}}_{s}$ and $\hat{S}_{a}^{\sigma}$ be as in Lemma B.5 (for the case $\sigma(\mathbf{u}, \mathbf{y})=\mathbf{u}^{T} \mathbf{y}$ ). It follows from Lemma B.5 that $X_{-}=T^{T} \operatorname{diag}\left(\hat{X}_{-} \quad 0\right) T$ where $\hat{X}_{-} \in$ $\mathbb{R}_{s}^{\hat{d} \times \hat{d}}$ with $\frac{1}{2} \hat{\mathbf{x}}_{0}^{T} \hat{X}_{-} \hat{\mathbf{x}}_{0}=\hat{S}_{a}^{\sigma_{p}}\left(\hat{\mathbf{x}}_{0}\right)$ for all $\hat{\mathbf{x}}_{0} \in \mathbb{R}^{\hat{d}}$. From case (ii), $\hat{X}_{-}$is the unique real matrix satisfying (a) $\hat{X}_{-} \geq 0$; and (b) there exist real matrices $\hat{L}, \hat{W}$ such that

(b1) $-A_{11}^{T} \hat{X}_{-}-\hat{X}_{-} A_{11}=\hat{L}^{T} \hat{L}, \quad C_{1}-B_{1}^{T} \hat{X}_{-}=\hat{W}^{T} \hat{L}, \quad$ and $D+D^{T}=\hat{W}^{T} \hat{W} ;$ and

(b2) $\hat{W}+\hat{L}\left(\xi I-A_{11}\right)^{-1} B_{1}$ is a spectral factor of $H+H^{\star}$.

Then, with $L:=\left[\begin{array}{ll}\hat{L} & 0\end{array}\right] T$, and $W:=\hat{W}$, it can be verified that condition 2$]$ of the present theorem statement holds. Also, if 
$X_{-}, L$ and $W$ are real matrices satisfying condition 2 , then $X_{-}=T^{T} \operatorname{diag}\left(\hat{X}_{-} \quad 0\right) T$ for some $0 \leq \hat{X}_{-} \in \mathbb{R}_{s}^{\hat{d} \times \hat{d}}$ with

$$
\begin{gathered}
{\left[\begin{array}{cc}
\left(T^{-1}\right)^{T} & 0 \\
0 & I
\end{array}\right]\left[\begin{array}{cc}
-A^{T} X_{-}-X_{-} A & C^{T}-X_{-} B \\
C-B^{T} X_{-} & D+D^{T}
\end{array}\right]\left[\begin{array}{cc}
\left(T^{-1}\right) & 0 \\
0 & I
\end{array}\right]} \\
\quad=\left[\begin{array}{cc|c}
-A_{11}^{T} \hat{X}_{-}-\hat{X}_{-} A_{11} & 0 & C_{1}^{T}-\hat{X}_{-} B_{1} \\
0 & 0 & 0 \\
\hline C_{1}-B_{1}^{T} \hat{X}_{-} & 0 & D+D^{T}
\end{array}\right]
\end{gathered}
$$

This implies that $L=\left[\begin{array}{ll}\hat{L} & 0\end{array}\right] T$, and $W=\hat{W}$ where $\hat{L}$ and $\hat{W}$ satisfy the aforementioned conditions (b1) and (b2). Then, from case (ii) and Lemma B.5. $S_{a}^{\sigma_{p}}\left(\mathbf{x}_{0}\right)=\hat{S}_{a}^{\sigma_{p}}\left(T_{1} \mathbf{x}_{0}\right)=$ $\frac{1}{2} \mathbf{x}_{0}^{T} T_{1}^{T} \hat{X}_{-} T_{1} \mathbf{x}_{0}=\frac{1}{2} \mathbf{x}_{0}^{T} X_{-} \mathbf{x}_{0}$ for all $\mathbf{x}_{0} \in \mathbb{R}^{d}$.

We conclude this section with a remark about computing the optimal control.

Remark 16: If $\mathcal{B}_{s}$ in (1) satisfies $m=n$ and $D+D^{T}>0$, and $A_{\Gamma}\left(X_{-}\right)$in Theorem 112 satisfies $\operatorname{spec}\left(A_{\Gamma}\left(X_{-}\right)\right) \in \mathbb{C}_{-}$, then $\mathbf{u}:=-\left(D+D^{T}\right)^{-1}\left(C-B^{T} X_{-}\right) \mathbf{x}$ and $(\mathbf{u}, \mathbf{y}, \mathbf{x}) \in$ $\mathcal{B}_{s}$ imply $\int_{t_{0}}^{t_{1}}-\left(\mathbf{u}^{T} \mathbf{y}\right)(t) d t=-\frac{1}{2}\left[\left(\mathbf{x}^{T} X_{-} \mathbf{x}\right)(t)\right]_{t_{0}}^{t_{1}}$ and $\frac{d \mathbf{x}}{d t}=A_{\Gamma}\left(X_{-}\right) \mathbf{x}$. Thus, if $\mathbf{x}\left(t_{0}\right)=\mathbf{x}_{0}$, then $\int_{t_{0}}^{\infty}-\left(\mathbf{u}^{T} \mathbf{y}\right)(t) d t=$ $S_{a}^{\sigma_{p}}\left(\mathbf{x}_{0}\right)$.

If, on the other hand, $D+D^{T}$ is singular or $\operatorname{spec}\left(A_{\Gamma}\left(X_{-}\right)\right) \quad \notin \mathbb{C}_{-}$, then there still exists a linear state feedback law such that, with $\mathbf{x}_{0}:=\mathbf{x}\left(t_{0}\right)$, then $\int_{t_{0}}^{\infty}-\left(\mathbf{u}^{T} \mathbf{y}\right)(t) d t$ comes arbitrarily close to the supremum $S_{a}^{\sigma_{p}}\left(\mathbf{x}_{0}\right)$. This can be constructed as follows. First, it follows from note A.1 and Lemma B.5 that no generality is lost in assuming $(C, A)$ is observable. We then let $\epsilon>0$, and we note that $(I+\epsilon D)$ is necessarily nonsingular. We define

$$
\begin{gathered}
A_{\epsilon}:=A-B(I+\epsilon D)^{-1} \epsilon C, B_{\epsilon}:=B(I+\epsilon D)^{-1} \sqrt{1+\epsilon^{2}}, \\
C_{\epsilon}:=\frac{\left(1-\epsilon^{2}\right)}{\sqrt{1+\epsilon^{2}}}(I+\epsilon D)^{-1} C, D_{\epsilon}:=(D+\epsilon I)(I+\epsilon D)^{-1}, \text { and } \\
\mathcal{B}_{s}^{\epsilon}:=\left\{\left(\mathbf{u}_{\epsilon}, \mathbf{y}_{\epsilon}, \mathbf{x}\right) \in \mathcal{L}_{2}^{\text {loc }}\left(\mathbb{R}, \mathbb{R}^{n}\right) \times \mathcal{L}_{2}^{\text {loc }}\left(\mathbb{R}, \mathbb{R}^{n}\right) \times \mathcal{L}_{2}^{\text {loc }}\left(\mathbb{R}, \mathbb{R}^{d}\right) \mid\right. \\
\left.\quad \frac{d \mathbf{x}}{d t}=A_{\epsilon} \mathbf{x}+B_{\epsilon} \mathbf{u}_{\epsilon} \text { and } \mathbf{y}_{\epsilon}=C_{\epsilon} \mathbf{x}+D_{\epsilon} \mathbf{u}_{\epsilon}\right\},
\end{gathered}
$$

so $\mathbf{u}_{\epsilon}=(\mathbf{u}+\epsilon \mathbf{y}) / \sqrt{1+\epsilon^{2}}$ and $\mathbf{y}_{\epsilon}=(\mathbf{y}+\epsilon \mathbf{u}) / \sqrt{1+\epsilon^{2}}$ satisfy

$$
\begin{aligned}
\int_{t_{0}}^{t_{1}}\left(\mathbf{u}_{\epsilon}^{T} \mathbf{y}_{\epsilon}\right)(t) d t= & \int_{t_{0}}^{t_{1}}\left(\mathbf{u}^{T} \mathbf{y}\right)(t) d t \\
& +\frac{\epsilon}{\sqrt{1-\epsilon^{2}}} \int_{t_{0}}^{t_{1}}\left(\mathbf{u}_{\epsilon}^{T} \mathbf{u}_{\epsilon}+\mathbf{y}_{\epsilon}^{T} \mathbf{y}_{\epsilon}\right)(t) d t,
\end{aligned}
$$

and $(\mathbf{u}, \mathbf{y}, \mathbf{x}) \in \mathcal{B}_{s}$ if and only if $\left(\mathbf{u}_{\epsilon}, \mathbf{y}_{\epsilon}, \mathbf{x}\right) \in \mathcal{B}_{s}^{\epsilon}$. Also, with $H(\xi):=D+C(\xi I-A)^{-1} B$ and $H_{\epsilon}(\xi):=$ $D_{\epsilon}+C_{\epsilon}\left(\xi I-A_{\epsilon}\right)^{-1} B_{\epsilon}$, then $H_{\epsilon}=(H+\epsilon I)(I+\epsilon H)^{-1}$. It can then be verified that $H_{\epsilon}(j \omega)+H_{\epsilon}(-j \omega)^{T}>0$ for all $\omega \in \mathbb{R}$, $D_{\epsilon}+D_{\epsilon}^{T}>0$, and $H_{\epsilon}$ has no poles in $\overline{\mathbb{C}}_{+}$. Since, in addition, $(C, A)$ is observable and $(A, B)$ is stabilizable, then it can be shown that $\operatorname{spec}\left(A_{\epsilon}\right) \in \mathbb{C}_{-}$. It then follows from [11] that there exists $X_{-}^{\epsilon} \in \mathbb{R}_{s}^{d \times d}$ such that

$-A_{\epsilon}^{T} X_{-}^{\epsilon}-X_{-}^{\epsilon} A_{\epsilon}-\left(C_{\epsilon}^{T}-X_{-}^{\epsilon} B_{\epsilon}\right)\left(D_{\epsilon}+D_{\epsilon}^{T}\right)^{-1}\left(C_{\epsilon}-B_{\epsilon}^{T} X_{-}^{\epsilon}\right)=0$, and $\operatorname{spec}\left(A_{\epsilon}-B_{\epsilon}\left(D_{\epsilon}+D_{\epsilon}^{T}\right)^{-1}\left(C_{\epsilon}-B_{\epsilon}^{T} X_{-}^{\epsilon}\right)\right) \in \mathbb{C}_{-}$,

and it follows that if $\mathbf{u}_{\epsilon}:=-\left(D_{\epsilon}+D_{\epsilon}^{T}\right)^{-1}\left(C_{\epsilon}-B_{\epsilon}^{T} X_{-}^{\epsilon}\right) \mathbf{x}$ and $\left(\mathbf{u}_{\epsilon}, \mathbf{y}_{\epsilon}, \mathbf{x}\right) \in \mathcal{B}_{s}^{\epsilon}$, then $\mathbf{x}(t) \rightarrow 0$ as $t \rightarrow \infty$, and $\int_{t_{0}}^{\infty}-\left(\mathbf{u}_{\epsilon}^{T} \mathbf{y}_{\epsilon}\right)(t) d t=\frac{1}{2} \mathbf{x}\left(t_{0}\right)^{T} X_{-}^{\epsilon} \mathbf{x}\left(t_{0}\right)$. Thus, if $\mathbf{u}=(I+$ $\epsilon D)^{-1}\left(\sqrt{1+\epsilon^{2}} \mathbf{u}_{\epsilon}-\epsilon C \mathbf{x}\right)$ and $(\mathbf{u}, \mathbf{y}, \mathbf{x}) \in \mathcal{B}_{s}$, then $\mathbf{u}_{\epsilon}=$ $(\mathbf{u}+\epsilon \mathbf{y}) / \sqrt{1+\epsilon^{2}}$ and $\mathbf{y}_{\epsilon}=(\mathbf{y}+\epsilon \mathbf{u}) / \sqrt{1+\epsilon^{2}}$, so $\mathbf{x}(t) \rightarrow 0$ as $t \rightarrow \infty$ and $\int_{t_{0}}^{\infty}-\left(\mathbf{u}^{T} \mathbf{y}\right)(t) d t \geq \frac{1}{2} \mathbf{x}\left(t_{0}\right)^{T} X_{-}^{\epsilon} \mathbf{x}\left(t_{0}\right)$ by (21). Finally, it can be verified that $X_{-}^{\epsilon} \rightarrow X_{-}$as $\epsilon \rightarrow 0$, so $\int_{t_{0}}^{\infty}-\left(\mathbf{u}^{T} \mathbf{y}\right)(t) d t$ can be made arbitrarily close to the supremum $S_{a}^{\sigma_{p}}\left(\mathbf{x}_{0}\right)$ by taking $\epsilon$ sufficiently small.

A similar argument holds for non-expansive behaviors (considered in the next three sections). In this case, we let $A_{\epsilon}:=A, B_{\epsilon}:=B, C_{\epsilon}:=(1-\epsilon) C, D_{\epsilon}:=(1-\epsilon) D$.

\section{NON-EXPANSIVE SYSTEMS}

In addition to the results on passive systems, we also extend the famous bounded-real lemma to systems that are neither observable nor controllable. This lemma is concerned with non-expansive systems, defined as follows.

Definition 17 (Non-expansive system): Let $\mathcal{B}_{s}$ be as in (1). For any given $\mathbf{x}_{0} \in \mathbb{R}^{d}$, let

$$
\begin{array}{r}
\mathcal{E}_{+}^{\sigma_{g}}\left(\mathbf{x}_{0}\right)=\left\{\int_{t_{0}}^{t_{1}}\left(\mathbf{y}^{T} \mathbf{y}-\mathbf{u}^{T} \mathbf{u}\right)(t) d t \mid t_{1} \geq t_{0},(\mathbf{u}, \mathbf{y}, \mathbf{x}) \in \mathcal{B}_{s},\right. \\
\text { and } \left.\mathbf{x}\left(t_{0}\right)=\mathbf{x}_{0}\right\} .
\end{array}
$$

Then the available storage $S_{a}^{\sigma_{g}}$ satisfies (i) $S_{a}^{\sigma_{g}}\left(\mathbf{x}_{0}\right)=$ $\sup \left(\mathcal{E}_{+}^{\sigma_{g}}\left(\mathbf{x}_{0}\right)\right)$ if $\mathcal{E}_{+}^{\sigma_{g}}\left(\mathbf{x}_{0}\right)$ is bounded above; and (ii) $S_{a}^{\sigma_{g}}\left(\mathbf{x}_{0}\right)=\infty$ otherwise. If $S_{a}^{\sigma_{g}}\left(\mathbf{x}_{0}\right)<\infty$ for all $\mathbf{x}_{0} \in \mathbb{R}^{d}$, then $\mathcal{B}_{s}$ is called non-expansive.

In our results, the following new concept of a bounded-real pair plays a central role.

Definition 18 (Bounded-real pair): Let $P \in \mathbb{R}^{m \times n}[\xi]$ and $Q \in \mathbb{R}^{m \times m}[\xi]$. We call $(P, Q)$ a bounded-real pair if the following hold:

(a) $Q(\lambda) Q(\bar{\lambda})^{T}-P(\lambda) P(\bar{\lambda})^{T} \geq 0$ for all $\lambda \in \overline{\mathbb{C}}_{+}$.

(b) $\operatorname{rank}\left(\left[\begin{array}{ll}P & -Q\end{array}\right](\lambda)\right)=m$ for all $\lambda \in \overline{\mathbb{C}}_{+}$.

(c) If $\mathbf{p} \in \mathbb{R}^{m}[\xi]$ and $\lambda \in \mathbb{C}$ satisfy $\mathbf{p}^{T}\left(Q Q^{\star}-P P^{\star}\right)=0$ and $\mathbf{p}(\lambda)^{T}\left[\begin{array}{ll}P & -Q\end{array}\right](\lambda)=0$, then $\mathbf{p}(\lambda)=0$.

Remark 19: It can be shown that, if $(P, Q)$ is a boundedreal pair, then $Q$ is nonsingular and $\left\|Q^{-1} P\right\|_{\infty} \leq 1$. But the converse is not true. For example, if $P(\xi)=Q(\xi)=\xi+1$, then $\left\|Q^{-1} P\right\|_{\infty}=1$, and condition (b) in Definition 18 holds, but not condition (c), so $(P, Q)$ is not a bounded-real pair. $\triangle$

In this section, we provide necessary and sufficient conditions for a system to be non-expansive (in the absence of any controllability and observability assumptions). These relate (a) the existence of matrices $X \in \mathbb{R}_{s}^{d \times d}$ such that $X \geq 0$ and

$$
\Lambda(X):=\left[\begin{array}{cc}
-A^{T} X-X A-C^{T} C & -C^{T} D-X B \\
-D^{T} C-B^{T} X & I-D^{T} D
\end{array}\right]
$$

satisfies $\Lambda(X) \geq 0$; and (b) the bounded-real pair concept. Also, if $I-D^{T} D>0$, then, with the notation

$$
\begin{array}{r}
\Pi(X):=-A^{T} X-X A-C^{T} C \\
\quad-\left(C^{T} D+X B\right)\left(I-D^{T} D\right)^{-1}\left(D^{T} C+B^{T} X\right), \\
\text { and } A_{\Pi}(X):=A+B\left(I-D^{T} D\right)^{-1}\left(D^{T} C+B^{T} X\right),
\end{array}
$$

conditions (a)-(b) also relate to the spectral properties of $A_{\Pi}(X)$ for solutions $X$ to the $\operatorname{ARE} \Pi(X)=0$. The results in this section are presented in the next three theorems, which we prove in Sections VII VIII

Theorem 20: Let $\mathcal{B}_{s}, \mathcal{B}_{s}^{(\mathbf{u}, \mathbf{y})}, V_{o}$ and $\Lambda$ be as in (11), (4), (6) and (22), respectively; and let $S_{a}^{\sigma_{g}}$ be as in Definition 17. The following are equivalent: 
1. $S_{a}^{\sigma_{g}}\left(\mathbf{x}_{0}\right)<\infty$ for all $\mathbf{x}_{0} \in \mathbb{R}^{d}$ (i.e., $\mathcal{B}_{s}$ is non-expansive).

2. The external behavior $\mathcal{B}_{s}^{(\mathbf{u}, \mathbf{y})}$ takes the form of (5), where $(P, Q)$ is a bounded-real pair.

3. There exists $X \in \mathbb{R}_{s}^{d \times d}$ such that $X \geq 0$ and $\Lambda(X) \geq 0$.

4. $S_{a}^{\sigma_{g}}\left(\mathbf{x}_{0}\right)=\mathbf{x}_{0}^{T} X_{-} \mathbf{x}_{0}$, where $X_{-} \in \mathbb{R}_{s}^{d \times d}$ satisfies (i) $X_{-} \geq 0$; (ii) $\Lambda\left(X_{-}\right) \geq 0$; (iii) if $\mathbf{z} \in \mathbb{R}^{d}$, then $V_{o} \mathbf{z}=0 \Longleftrightarrow X_{-} \mathbf{z}=0$; and (iv) if $X \in \mathbb{R}_{s}^{d \times d}$ satisfies $X \geq 0$ and $\Lambda(X) \geq 0$, then $X_{-} \leq X$.

Moreover, if $(C, A)$ is observable and the above conditions hold, then (i) $\operatorname{spec}(A) \in \mathbb{C}_{-}$; and (ii) if $X \in \mathbb{R}_{s}^{d \times d}$ satisfies $\Lambda(X) \geq 0$, then $X_{-} \leq X$.

Remark 21: From [4, Theorems 3-6], if $(A, B)$ is controllable, then (i) for a system to be non-expansive it is necessary and sufficient for the $\mathcal{H}_{\infty}$ norm of the system's transfer function to be bounded above by one; and (ii) the set of solutions to the LMI in the bounded-real lemma (condition 3 in Theorem 201 is bounded. However, both of these conditions can fail to hold when $(A, B)$ is not controllable.

Theorem 22 provides an explicit solution to the optimal control problem in Definition 17 in the case $I-D^{T} D>0$.

Theorem 22: Let $\mathcal{B}_{s}, V_{o}, H, \Pi$ and $A_{\Pi}$ be as in (1), (6), (8), (23) and (24), respectively; let $S_{a}^{\sigma_{g}}$ be as in Definition 17, and let $I-D^{T} D>0$. The following are equivalent

1. $S_{a}^{\sigma_{g}}\left(\mathbf{x}_{0}\right)<\infty$ for all $\mathbf{x}_{0} \in \mathbb{R}^{d}$ (i.e., $\mathcal{B}_{s}$ is non-expansive).

2. There exists $X_{-} \in \mathbb{R}_{s}^{d \times d}$ satisfying (i) $X_{-} \geq 0$; (ii) $\Pi\left(X_{-}\right)=0$; (iii) if $\mathbf{z} \in \mathbb{R}^{d}$ satisfies $V_{o} \mathbf{z}=0$, then $X_{-} \mathbf{z}=0$; and (iv) if $\lambda \in \mathbb{C}_{+}$and $\mathbf{z} \in \mathbb{C}^{d}$ satisfy $A_{\Pi}\left(X_{-}\right) \mathbf{z}=\lambda \mathbf{z}$, then $V_{o} \mathbf{z}=0$.

Moreover, if these conditions hold, then $S_{a}^{\sigma_{g}}\left(\mathbf{x}_{0}\right)=\mathbf{x}_{0}^{T} X_{-} \mathbf{x}_{0}$.

Theorem 23 solves the optimal control problem in Definition 17 in the general case.

Theorem 23: Let $\mathcal{B}_{s}, V_{o}$ and $H$ be as in (1), (6) and (8), respectively; and let $S_{a}^{\sigma_{g}}$ be as in Definition 17. The following are equivalent:

1. $S_{a}^{\sigma_{g}}\left(\mathbf{x}_{0}\right)<\infty$ for all $\mathbf{x}_{0} \in \mathbb{R}^{d}$ (i.e., $\mathcal{B}_{s}$ is non-expansive).

2. There exists $X_{-} \in \mathbb{R}_{s}^{d \times d}$ satisfying (i) $X_{-} \geq 0$; (ii) if $\mathbf{z} \in \mathbb{R}^{d}$ satisfies $V_{o} \mathbf{z}=0$, then $X_{-} \mathbf{z}=0$; and (iii) there exist real matrices $L$ and $W$ such that

(iiia) $-A^{T} X_{-}-X_{-} A-C^{T} C=L^{T} L,-D^{T} C-B^{T} X_{-}=$ $W^{T} L$, and $I-D^{T} D=W^{T} W$; and

(iiib) $Z(\xi):=W+L(\xi I-A)^{-1} B$ is a spectral factor of $I-H^{\star} H$.

Moreover, if these conditions hold, then $S_{a}^{\sigma_{g}}\left(\mathbf{x}_{0}\right)=\mathbf{x}_{0}^{T} X_{-} \mathbf{x}_{0}$.

Remark 24: As is the case with the positive-real lemma, there have been many notable attempts to relax the controllability and observability assumptions in the bounded-real lemma. A particularly well known result is the so-called strictly bounded-real lemma [6, Lemma 5.6.5]. This lemma proves that, if $\mathcal{B}_{s}$ is as in (1) and $\operatorname{spec}(A) \in \mathbb{C}_{-}$, and $H, \Pi$ and $A_{\Pi}$ are as in (8), (23) and (24) then $\|H\|_{\infty}<1$ if and only if $I-D^{T} D>0$ and there exists $X \geq 0$ such that $\Pi(X)=0$ and $\operatorname{spec}\left(A_{\Pi}(X)\right) \in \mathbb{C}_{-}$.

\section{NON-EXPANSIVE SYSTEMS AND THE AVAILABLE STORAGE}

To prove Theorem 20, we will employ transformations that relate non-expansive and passive systems, and similar transformations that relate positive-real and bounded-real pairs.
Proof of Theorem 20. We will first show the two chains of implications $1 \Rightarrow 4 \Rightarrow 3 \Rightarrow 1$ and $4 \Rightarrow 2 \Rightarrow 3$

$\mathbf{1} \Rightarrow \mathbf{4} \Rightarrow \mathbf{3} \Rightarrow \mathbf{1} \quad$ First, let $\mathrm{z} \in \mathbb{R}^{d}$, and let $\tilde{\mathbf{x}}(t)=e^{A\left(t-t_{0}\right)} \mathbf{z}$ for all $t \in \mathbb{R}, \tilde{\mathbf{u}}=0$, and $\tilde{\mathbf{y}}=C \tilde{\mathbf{x}}$. Then $(\tilde{\mathbf{u}}, \tilde{\mathbf{y}}, \tilde{\mathbf{x}}) \in \mathcal{B}_{s}, \tilde{\mathbf{x}}\left(t_{0}\right)=\mathbf{z}$, and $\int_{t_{0}}^{t_{1}}\left(\tilde{\mathbf{y}}^{T} \tilde{\mathbf{y}}-\tilde{\mathbf{u}}^{T} \tilde{\mathbf{u}}\right)(t) d t \geq 0$. Second, note that $\int_{t_{0}}^{t_{1}}\left(\mathbf{u}^{T} \mathbf{u}-\mathbf{y}^{T} \mathbf{y}\right)(t) d t-\left[\mathbf{x}^{T} X \mathbf{x}\right]_{t_{0}}^{t_{1}}=$ $\int_{t_{0}}^{t_{1}}\left(\left[\begin{array}{ll}\mathbf{x}^{T} & \mathbf{u}^{T}\end{array}\right] \Lambda(X) \operatorname{col}(\mathbf{x} \quad \mathbf{u})\right)(t) d t$. With these two observations, the present implications can be shown in a similar manner to the corresponding implications in Theorem 10.

$2 \Rightarrow$ 3. Consider the observer staircase form (see note A[1), and let

$$
\hat{\Lambda}(\hat{X}):=\left[\begin{array}{cc}
-\hat{A}_{11}^{T} \hat{X}-\hat{X} \hat{A}_{11}-\hat{C}_{1}^{T} \hat{C}_{1} & -\hat{C}_{1}^{T} \hat{D}-\hat{X} \hat{B}_{1} \\
-\hat{D}^{T} \hat{C}_{1}-\hat{B}_{1}^{T} \hat{X} & I-\hat{D}^{T} \hat{D}
\end{array}\right] .
$$

If there exists $\hat{X} \in \mathbb{R}_{s}^{\hat{d} \times \hat{d}}$ satisfying $\hat{X} \geq 0$ and $\hat{\Lambda}(\hat{X}) \geq 0$, then $X:=T^{T} \operatorname{diag}(\hat{X} \quad 0) T$ satisfies $X \geq 0$ and $\Lambda(X) \geq 0$. Thus, it suffices to prove this implication for the case with $(C, A)$ observable. We will prove this for the cases: (i) $m=n$, (ii) $n<m$, and (iii) $m<n$.

Case (i): $\mathbf{m}=\mathbf{n}$. Let $\mathcal{A}(\xi):=\xi I-A$, and let $M, N, U, V, E, F$ and $G$ be polynomial matrices satisfying conditions (a) and (b) in note $\mathrm{A} / 2$. From note $\mathrm{C} / 4$, there exists a signature matrix $\Sigma$ and matrices

$$
\hat{Q}:=\frac{1}{2}(Q-P \Sigma) \text { and } \hat{P}:=\frac{1}{2}(P \Sigma+Q)
$$

such that $\hat{Q}$ is nonsingular and $\hat{Q}^{-1} \hat{P}$ is proper. Now, let $D:=$ $\lim _{\xi \rightarrow \infty}\left(Q^{-1} P(\xi)\right)$ and $\hat{D}:=\lim _{\xi \rightarrow \infty}\left(\hat{Q}^{-1} \hat{P}(\xi)\right)$. Note that $\left(I-Q^{-1} P \Sigma\right) \hat{Q}^{-1} \hat{P}=I+Q^{-1} P \Sigma$, so by taking the limit as $\xi \rightarrow \infty$ we obtain $(I-D \Sigma) \hat{D}=I+D \Sigma$. Thus, if $\mathbf{z} \in \mathbb{R}^{m}$ and $\mathbf{z}^{T}(I-D \Sigma)=0$, then $\mathbf{z}^{T}(I+D \Sigma)=0$, so $\mathbf{z}=0$. Hence, $(I-D \Sigma)$ is nonsingular, and

$$
\hat{D}=(I-D \Sigma)^{-1}(I+D \Sigma)=2(I-D \Sigma)^{-1}-I .
$$

Now, let

$$
\left[\begin{array}{cc}
\hat{M} & \hat{N} \\
\hat{U} & \hat{V}
\end{array}\right]:=\left[\begin{array}{cc}
\frac{1}{2} I & 0 \\
0 & \frac{1}{\sqrt{2}} I
\end{array}\right]\left[\begin{array}{cc}
M & N \\
U & V
\end{array}\right]\left[\begin{array}{cc}
I-D \Sigma & 0 \\
-B \Sigma & \sqrt{2} I
\end{array}\right],
$$

so all of the above matrices are unimodular. Then, with

$$
\begin{aligned}
& \hat{A}:=A+B \Sigma(I-D \Sigma)^{-1} C, \quad \hat{B}:=\sqrt{2} B \Sigma(I-D \Sigma)^{-1}, \\
& \hat{C}:=\sqrt{2}(I-D \Sigma)^{-1} C, \hat{\mathcal{A}}(\xi):=\xi I-\hat{A} \\
& \hat{E}:=\frac{1}{\sqrt{2}}(E \Sigma-F), \text { and } \hat{F}:=\frac{1}{\sqrt{2}}(E \Sigma+F)
\end{aligned}
$$

it can be verified that $(\hat{C}, \hat{A})$ is observable, and

$$
\left[\begin{array}{cc}
\hat{M} & \hat{N} \\
\hat{U} & \hat{V}
\end{array}\right]\left[\begin{array}{ccc}
-\hat{D} & I & -\hat{C} \\
-\hat{B} & 0 & \hat{\mathcal{A}}
\end{array}\right]=\left[\begin{array}{ccc}
-\hat{P} & \hat{Q} & 0 \\
-\hat{E} & -\hat{F} & I
\end{array}\right] .
$$

Hence, $(\hat{A}, \hat{B}, \hat{C}, \hat{D})$ is an observable realization for $(\hat{P}, \hat{Q})$ (see note $\mathrm{A} / 2)$. Since $\hat{Q}:=\frac{1}{2}(Q-P \Sigma)$ and $\hat{P}:=\frac{1}{2}(P \Sigma+Q)$, then it follows from notes $\mathrm{C} / 2, \mathrm{C} / 4$ that $(\hat{P}, \hat{Q})$ is a positivereal pair. Thus, from Lemma 1 and Theorem 10 , there exists $X \in \mathbb{R}_{s}^{d \times d}$ such that $X>0$ and

$$
\hat{\Omega}(X):=\left[\begin{array}{cc}
-\hat{A}^{T} X-X \hat{A} & \hat{C}^{T}-X \hat{B} \\
\hat{C}-\hat{B}^{T} X & \hat{D}+\hat{D}^{T}
\end{array}\right]
$$


satisfies $\hat{\Omega}(X) \geq 0$. Furthermore, with

$$
S=\left[\begin{array}{cc}
I & 0 \\
-\frac{1}{\sqrt{2}} C & \frac{1}{\sqrt{2}}(I-D \Sigma) \Sigma
\end{array}\right],
$$

then it can be verified that $S^{T} \hat{\Omega}(X) S=\Lambda(X)$, which is nonnegative definite since $\hat{\Omega}(X)$ is. This proves case (i).

Case (ii): $\mathbf{m}>\mathbf{n} . \quad$ Let $\hat{P}:=\left[\begin{array}{ll}P & 0_{m \times(m-n)}\end{array}\right]$ and $\hat{Q}:=Q$. It is easily shown from note Cl1 that $(\hat{P}, \hat{Q})$ is a boundedreal pair. Also, with $\hat{A}=A, \hat{B}=\left[\begin{array}{ll}B & 0_{d \times(m-n)}\end{array}\right], \hat{C}=C$, and $\hat{D}=\left[\begin{array}{ll}D & 0_{m \times(m-n)}\end{array}\right]$, it can be verified that $(A, B, C, D)$ is an observable realization for $(P, Q)$ if and only if $(\hat{A}, \hat{B}, \hat{C}, \hat{D})$ is an observable realization for $(\hat{P}, \hat{Q})$. With

$$
\hat{\Lambda}(X):=\left[\begin{array}{cc}
-\hat{A}^{T} X-X \hat{A}-\hat{C}^{T} \hat{C} & -\hat{C}^{T} \hat{D}-X \hat{B} \\
-\hat{D}^{T} \hat{C}-\hat{B}^{T} X & I-\hat{D}^{T} \hat{D}
\end{array}\right],
$$

then $\hat{\Lambda}(X)=\operatorname{diag}(\Lambda(X) \quad I)$. From case (i), there exists $X>0$ such that $\hat{\Lambda}(X) \geq 0$. This $X$ also satisfies $\Lambda(X) \geq 0$.

Case (iii): $\mathbf{m}<\mathbf{n}$. . In this case, let $\hat{P}:=$ $\operatorname{col}\left(\begin{array}{ll}P & 0_{(n-m) \times n}\end{array}\right), \hat{Q}:=\operatorname{diag}\left(\begin{array}{ll}Q & I_{(n-m) \times(n-m)}\end{array}\right), \hat{A}=A$, $\hat{B}=B, \hat{C}=\operatorname{col}\left(\begin{array}{ll}C & 0_{(n-m) \times d}\end{array}\right)$, and $\hat{D}=\operatorname{col}\left(\begin{array}{ll}D & 0_{(n-m) \times n}\end{array}\right)$; and let $\Lambda(X)$ and $\hat{\Lambda}(X)$ be as in (22) and (31), respectively. Then $(\hat{P}, \hat{Q})$ is a bounded-real pair (this is easily shown from note C[1], $\Lambda(X)=\hat{\Lambda}(X)$, and the proof is similar to case (ii).

$4 \Rightarrow 2$. We will prove this for the two cases (i) $(C, A)$ observable; and (ii) $(C, A)$ not observable.

Case (i): $(\boldsymbol{C}, \boldsymbol{A})$ observable. We consider the case $m=$ $n$. The proofs for the cases $m>n$ and $m<n$ are then similar to the corresponding cases in the proof of $2 \Rightarrow 3$ Let $\Sigma, \hat{P}, \hat{Q}, \hat{A}, \hat{B}, \hat{C}, \hat{D}$, and $\hat{\Omega}(X)$ be as in case (i) of the proof of $2 \Rightarrow 3$. Then, from that proof, $(\hat{A}, \hat{B}, \hat{C}, \hat{D})$ is an observable realization of $(\hat{P}, \hat{Q})$, and $\hat{\Omega}\left(X_{-}\right) \geq 0$. Thus, $(\hat{P}, \hat{Q})$ is a positive-real pair by Theorem 10 so $(P, Q)$ is a bounded-real pair by notes $\mathrm{C}, 2, \mathrm{C}, 4$.

Case (ii): $(C, A)$ not observable. Consider the observer staircase form (see note Al1), and let $\hat{\Lambda}$ be as in (31). Then $X_{-}=T^{T} \operatorname{diag}\left(\hat{X}_{-} \quad 0\right) T$ where $\hat{X}_{-} \in \mathbb{R}_{s}^{\hat{d} \times \hat{d}}, \hat{\Lambda}\left(\hat{X}_{-}\right) \geq 0$ and $\hat{X}_{-} \geq 0$. Also, with $\hat{\mathcal{B}}_{s}$ as in note Al1 then $\mathcal{B}_{s}^{(\mathbf{u}, \mathbf{y})}=$ $\hat{\mathcal{B}}_{s}^{(\mathbf{u}, \mathbf{y})}$ as shown in that note. Condition 2 then follows from case (i).

It remains to prove conditions (i)-(ii) in the final paragraph of the present theorem statement. To see (i), let $\lambda \in \overline{\mathbb{C}}_{+}$and $\mathbf{z} \in \mathbb{C}^{d}$ satisfy $(\lambda I-A) \mathbf{z}=0$, and note that $\overline{\mathbf{z}}^{T}\left(A^{T} X+\right.$ $X A) \mathbf{z}=(\lambda+\bar{\lambda}) \overline{\mathbf{z}}^{T} X \mathbf{z}$. Since $-A^{T} X-X A-C^{T} C \geq 0$, then $\overline{\mathbf{z}}^{T} C^{T} C \mathbf{z} \leq-2 \Re(\lambda) \overline{\mathbf{z}}^{T} X \mathbf{z} \leq 0$, so $C \mathbf{z}=0$. If $(C, A)$ is observable, then $\mathbf{z}=0$, so $\operatorname{spec}(A) \in \mathbb{C}_{-}$. The proof of condition (ii) is similar to the corresponding condition in Theorem 10, using the observations in the second paragraph of this proof.

\section{EXPLICIT CHARACTERISATION OF THE AVAILABLE STORAGE FOR A NON-EXPANSIVE SYSTEM}

This section contains the proofs of Theorems 22 and 23 . The proofs provide methods for calculating the available storage for a non-expansive system by using the results in Section $\mathrm{V}$

Proof of Theorem $22.2 \Rightarrow 1$ This follows from Theorem 20, since $X_{-} \geq 0$ and $\Lambda\left(X_{-}\right) \geq 0$.
$1 \Rightarrow 2$ First, we note from Theorem 20 that $(P, Q)$ is a bounded-real pair since $S_{a}^{\sigma_{g}}\left(\mathbf{x}_{0}\right)<\infty$. We will show that this implies condition 2 for the cases: (i) $(C, A)$ observable and $m=n$; (ii) $(C, A)$ observable and $m>n$; (iii) $(C, A)$ observable and $m<n$; then finally (iv) $(C, A)$ not observable.

Case (i) $(C, A)$ observable, $\mathbf{m}=\mathbf{n}$. Let $\Sigma, \hat{P}, \hat{Q}, \hat{A}, \hat{B}, \hat{C}$, and $\hat{D}$ be as in case (i) in the proof of $2 \Rightarrow 3$ in Theorem 20 From that proof, $(\hat{P}, \hat{Q})$ is a positive-real pair, and $(\hat{A}, \hat{B}, \hat{C}, \hat{D})$ is an observable realization of $(\hat{P}, \hat{Q})$. From Theorem 12] with the notation

$\hat{\Gamma}(X):=-\hat{A}^{T} X-X \hat{A}-\left(\hat{C}^{T}-X \hat{B}\right)\left(\hat{D}+\hat{D}^{T}\right)^{-1}\left(\hat{C}-\hat{B}^{T} X\right)$, and $A_{\hat{\Gamma}}(X):=\hat{A}-\hat{B}\left(\hat{D}+\hat{D}^{T}\right)^{-1}\left(\hat{C}-\hat{B}^{T} X\right)$,

there exists $X \in \mathbb{R}_{s}^{d \times d}$ such that $X \geq 0, \hat{\Gamma}(X)=0$, and $\operatorname{spec}\left(A_{\hat{\Gamma}}(X)\right) \in \overline{\mathbb{C}}_{-}$. It can then be verified that $\Pi(X)=$ $\hat{\Gamma}(X)$ and $A_{\Pi}(X)=A_{\hat{\Gamma}}(X)$, so condition 2 holds.

Case (ii) $(\boldsymbol{C}, \boldsymbol{A})$ observable, $\mathbf{m}>\mathbf{n}$. Let $\hat{P}, \hat{Q}, \hat{A}, \hat{B}, \hat{C}$, and $\hat{D}$ be as in case (ii) in the proof of $2 \Rightarrow 3$ in Theorem 20, so $(\hat{P}, \hat{Q})$ is a bounded-real pair, and $(A, B, C, D)$ is an observable realization for $(P, Q)$ if and only if $(\hat{A}, \hat{B}, \hat{C}, \hat{D})$ is an observable realization for $(\hat{P}, \hat{Q})$. Also, let

$$
\begin{aligned}
& \hat{\Pi}(X):=-\hat{A}^{T} X-X \hat{A}-\hat{C}^{T} \hat{C} \\
& \quad-\left(\hat{C}^{T} \hat{D}+X \hat{B}\right)\left(I-\hat{D}^{T} \hat{D}\right)^{-1}\left(\hat{D}^{T} \hat{C}+\hat{B}^{T} X\right), \\
& \text { and } \hat{A}_{\hat{\Pi}}(X):=\hat{A}+\hat{B}\left(I-\hat{D}^{T} \hat{D}\right)^{-1}\left(\hat{D}^{T} \hat{C}+\hat{B}^{T} X\right)
\end{aligned}
$$

It can be verified that $\hat{\Pi}(X)=\Pi(X)$ and $\hat{A}_{\hat{\Pi}}(X)=A_{\Pi}(X)$, so this case follows from case (i).

Case (iii) $(C, A)$ observable, $\mathbf{m}<\mathbf{n}$. In this case, we let $\hat{P}, \hat{Q}, \hat{A}, \hat{B}, \hat{C}$, and $\hat{D}$ be as in case (iii) in the proof of $2 \Rightarrow 3$ in Theorem 20. Then, with $\hat{\Pi}(X)$ and $\hat{A}_{\hat{\Pi}}(X)$ as in (32)-(33), we obtain $\hat{\Pi}(X)=\Pi(X)$ and $\hat{A}_{\hat{\Pi}}(X)=A_{\Pi}(X)$. The proof then follows the argument in case (ii).

Case (iv) $(C, A)$ not observable. This can be proved in the manner of case (ii) in the proof of $1 \Rightarrow 2$ in Theorem 12 .

Finally, with a similar proof to the corresponding implication in Theorem 12, we find that if $X_{-}$satisfies condition 2 of the present theorem, then $S_{a}^{\sigma_{g}}\left(\mathbf{x}_{0}\right)=\mathbf{x}_{0}^{T} X_{-} \mathbf{x}_{0}$.

Proof of Theorem 23. $2 \Rightarrow$ 1, This follows from Theorem [20, since $X_{-} \geq 0$ and $\Lambda\left(X_{-}\right) \geq 0$.

For the remainder of the proof, we let $(C, A)$ be observable and $m=n$. The cases $m>n$ and $m<n$ can be shown by augmenting to the case $m=n$ as in the proof of Theorem 22. The case $(C, A)$ not observable can be shown with a similar argument to the corresponding implication in Theorem 13 .

$1 \Rightarrow 2$ Since $S_{a}^{\sigma_{g}}\left(\mathrm{x}_{0}\right)<\infty$ for all $\mathrm{x}_{0} \in \mathbb{R}^{d}$, then $(P, Q)$ is a bounded-real pair by Theorem [20, Next, let $\Sigma, \hat{P}, \hat{Q}, \hat{A}, \hat{B}, \hat{C}$, and $\hat{D}$ be as in case (i) in the proof of 2 $\Rightarrow 3$ in Theorem 20 (so $I-D \Sigma$ is nonsingular and $(\hat{C}, \hat{A}$ ) is observable), and let $\hat{H}(\xi):=\hat{D}+\hat{C}(\xi I-\hat{A})^{-1} \hat{B}$. Then $(\hat{P}, \hat{Q})$ is a positive-real pair, so from Theorems 10,13 there exist real matrices $X_{-}, \hat{L}$, and $\hat{W}$ with $X_{-} \geq 0$ such that

(a) $-\hat{A}^{T} X_{-}-X_{-} \hat{A}=\hat{L}^{T} \hat{L}, \hat{C}-\hat{B}^{T} X_{-}=\hat{W}^{T} \hat{L}, \hat{D}+\hat{D}^{T}=$ $\hat{W}^{T} \hat{W} ;$ and

(b) $\hat{Z}(\xi):=\hat{W}+\hat{L}(\xi I-\hat{A})^{-1} \hat{B}$ is a spectral factor of $\hat{H}+\hat{H}^{\star}$. 
Then, let $L:=\hat{L}-\frac{1}{\sqrt{2}} \hat{W} C$ and $W:=\frac{1}{\sqrt{2}} \hat{W}(I-D \Sigma) \Sigma$, and it can be verified that condition (iiia) holds. Also,

$$
\left[\begin{array}{cc}
\lambda I-\hat{A} & -\hat{B} \\
\hat{L} & \hat{W}
\end{array}\right]\left[\begin{array}{cc}
I & 0 \\
-\frac{1}{\sqrt{2}} C & \frac{1}{\sqrt{2}}(I-D \Sigma) \Sigma
\end{array}\right]=\left[\begin{array}{cc}
\lambda I-A & -B \\
L & W
\end{array}\right] .
$$

From Theorem 10, $\operatorname{spec}(\hat{A}) \in \overline{\mathbb{C}}_{-}$. Also, from Theorem 20 $\operatorname{spec}(A) \in \mathbb{C}_{-}$. Since, in addition, $(I-D \Sigma)$ is nonsingular, then a similar argument to the proof of Lemma D.1 shows that $Z$ is a spectral factor of $I-H^{\star} H$.

Finally, we prove that if $X_{-}$satisfies condition 2 then $S_{a}^{\sigma_{g}}\left(\mathbf{x}_{0}\right)=\mathbf{x}_{0}^{T} X_{-} \mathbf{x}_{0}$. It suffices to show that $X_{-}$is uniquely determined by condition 2 To show this, we let $\Sigma, \hat{P}, \hat{Q}, \hat{A}, \hat{B}, \hat{C}, \hat{D}$ and $\hat{H}$ be as in the previous paragraph. Following that paragraph, if $X_{-}$satisfies condition 2, then $\hat{L}:=L+W \Sigma(I-D \Sigma)^{-1} C$ and $\hat{W}:=\sqrt{2} W \Sigma(I-D \Sigma)^{-1}$ satisfy the aforementioned conditions (a) and (b) From Theorem 13, these conditions uniquely determine $X_{-}$.

\section{REFERENCES}

[1] J. C. Willems, "Dissipative dynamical systems, Part I: General theory," Arch. Ration. Mech. Anal., vol. 45, pp. 321-351, 1972.

[2] — , "Dissipative dynamical systems, Part II: Linear systems with quadratic supply rates," Arch. Ration. Mech. Anal., vol. 45, pp. 352393, 1972.

[3] B. D. O. Anderson and S. Vongpanitlerd, Network Analysis and Synthesis. Upper Saddle River, NJ: Prentice-Hall, 1973.

[4] B. P. Molinari, "The time-invariant linear-quadratic optimal control problem," Automatica, vol. 13, pp. 345-357, 1977.

[5] T. H. Hughes, "A theory of passive linear systems with no assumptions," Automatica, vol. 86, pp. 87-97, 2017.

[6] B. Brogliato, R. Lozano, B. Maschke, and O. Egeland, Dissipative Systems Analysis and Control. London: Springer-Verlag, 2007.

[7] J. W. Polderman and J. C. Willems, Introduction to Mathematical Systems Theory: A Behavioral Approach. New York : Springer-Verlag, 1998.

[8] T. H. Hughes, "Behavioral realizations using companion matrices and the Smith form," SIAM Journal on Control Optim., vol. 54, no. 2, pp. 845-865, 2016

[9] M. K. Çamlibel, L. Iannelli, and F. Vasca, "Passivity and complementarity," Mathematical Programming, vol. 145, no. 1-2, pp. 531-563, 2014

[10] J. Collado, R. Lozano, and R. Johansson, "On Kalman-YakubovichPopov lemma for stabilizable systems," IEEE Trans. on Automatic Control, vol. 46, no. 7, pp. 1089-1093, July 2001.

[11] W. Sun, P. P. Khargonekar, and D. Shim, "Solution of the positive real control problem for linear time-invariant systems," IEEE Trans. on Automatic Control, vol. 39, no. 10, pp. 2034-2046, October 1994.

[12] L. Pandolfi, "An observation on the positive real lemma," Journal of Mathematical Analysis and Applications, vol. 255, no. 2, pp. 480-490, Mar 2001.

[13] S. Kunimatsu, K. Sang-Hoon, T. Fujii, and M. Ishitobi, "On positive real lemma for non-minimal realization systems," Proceedings of the 17th IFAC World Congress, Seoul, pp. 5868-5873, 2008.

[14] A. Ferrante and L. Pandolfi, "On the solvability of the positive real lemma equations," Systems and Control Letters, vol. 47, no. 3, pp. 211219, October 2002.

[15] A. Ferrante, "Positive real lemma: Necessary and sufficient conditions for the existence of solutions under virtually no assumptions," IEEE Trans. on Automatic Control, vol. 50, pp. 720-724, May 2005.

[16] M. K. Çamlibel, J. C. Willems, and M. N. Belur, "On the dissipativity of uncontrollable systems," in Proceedings of the 42nd IEEE Conference on Decision and Control, Hawaii, Dec. 2003.

[17] D. Pal and M. N. Belur, "Dissipativity of uncontrollable systems, storage functions, and Lyapunov functions," SIAM Journal on Control Optim., vol. 47, no. 6, pp. 2930-2966, 2008.

[18] B. D. O. Anderson and J. B. Moore, Linear Optimal Control. PrenticeHall, 1971.

[19] B. P. Molinari, "Nonnegativity of a quadratic functional," SIAM Journal on Control, vol. 13, no. 4, pp. 792-806, July 1975.

\section{APPENDIX A \\ OBSERVABLE REALIZATIONS OF BEHAVIORS}

In this appendix, we present several results on observable realizations which are used in the proofs of the main theorems. These results build on Lemmas 11 and 2

A 1 Let $\mathcal{B}_{s}$ and $V_{o}$ be as in (1) and (6); let the columns of $S_{2} \in \mathbb{R}^{d \times(d-\hat{d})}$ be a basis for the nullspace of $V_{o}$; let $S=$ $\left[\begin{array}{ll}S_{1} & S_{2}\end{array}\right]$ be nonsingular; and let $S^{-1}=: T=\operatorname{col}\left(\begin{array}{ll}T_{1} & T_{2}\end{array}\right)$ (partitioned compatibly with $S$ ). Then,

$$
\left[\begin{array}{l}
T_{1} \\
T_{2}
\end{array}\right] A\left[\begin{array}{ll}
S_{1} & S_{2}
\end{array}\right]=\left[\begin{array}{cc}
A_{11} & 0 \\
A_{21} & A_{22}
\end{array}\right], C\left[\begin{array}{ll}
S_{1} & S_{2}
\end{array}\right]=\left[\begin{array}{ll}
C_{1} & 0
\end{array}\right],
$$

and $\left(C_{1}, A_{11}\right)$ is observable [7, Corollary 5.3.14]. Furthermore, with the notation $B_{1}:=T_{1} B, B_{2}:=T_{2} B$, and

$$
\begin{gathered}
\hat{\mathcal{B}}_{s}=\left\{(\mathbf{u}, \mathbf{y}, \hat{\mathbf{x}}) \in \mathcal{L}_{2}^{\text {loc }}\left(\mathbb{R}, \mathbb{R}^{n}\right) \times \mathcal{L}_{2}^{\text {loc }}\left(\mathbb{R}, \mathbb{R}^{n}\right) \times \mathcal{L}_{2}^{\text {loc }}\left(\mathbb{R}, \mathbb{R}^{\hat{d}}\right)\right. \\
\text { such that } \left.\frac{d \hat{\mathbf{x}}}{d t}=A_{11} \hat{\mathbf{x}}+B_{1} \mathbf{u} \text { and } \mathbf{y}=C_{1} \hat{\mathbf{x}}+D \mathbf{u}\right\}
\end{gathered}
$$

then it is easily shown from the variation of the constants formula (2)-(3) that $\mathcal{B}_{s}^{(\mathbf{u}, \mathbf{y})}=\hat{\mathcal{B}}_{s}^{(\mathbf{u}, \mathbf{y})}$. Thus, if $P$ and $Q$ are as in Lemma 2, then it follows from Lemmas 142 that there exists an observable realization for $(P, Q)$.

A 2 Let $\mathcal{B}_{s}$ be as in (1) and $\mathcal{A}(\xi)=\xi I-A$. Then $(A, B, C, D)$ is an observable realization for $(P, Q)$ if and only if $P \in \mathbb{R}^{m \times n}[\xi], Q \in \mathbb{R}^{m \times m}[\xi]$, and there exist polynomial matrices $M, N, U, V, E, F$ and $G$ such that (a) conditions 1 and 2 of Lemma 11 hold; and (b) $G=I_{d}$. To see this, note from the final block column in condition 1 of Lemma 1 that, for any given $\lambda \in \mathbb{C}$ and $\mathbf{z} \in \mathbb{C}^{d}$, then $C \mathbf{z}=0$ and $(\lambda I-A) \mathbf{z}=0$ if and only if $G(\lambda) \mathbf{z}=0$. It then follows from [7. Theorem 5.3.7] that $G$ in Lemma 1 is unimodular if and only if $(C, A)$ is observable. Furthermore, if $G$ is unimodular, then by pre-multiplying both sides in condition 11 of Lemma 11 by diag $\left(I \quad G^{-1}\right)$ we obtain polynomial matrices satisfying conditions (a) and (b).

A 3 Let $P$ and $Q$ be as in Lemma 2 If $(A, B, C, D)$ and $(\hat{A}, \hat{B}, \hat{C}, \hat{D})$ are two observable realizations of $(P, Q)$, then there exists a nonsingular $T \in \mathbb{R}^{d \times d}$ such that

$$
\hat{A}=T A T^{-1}, \hat{B}=T B, \hat{C}=C T^{-1} \text {, and } \hat{D}=D .
$$

To see this, let $A \in \mathbb{R}^{d \times d}$ and $\hat{A} \in \mathbb{R}^{\hat{d} \times \hat{d}}$; let $V_{o}$ be as in (6); and let $\hat{V}_{o}:=\operatorname{col}\left(\hat{C} \hat{C} \hat{A} \cdots \hat{C} \hat{A}^{\hat{d}-1}\right)$. It follows from the variation of the constants formula (2)-(3) that, for any given $\mathbf{z} \in \mathbb{R}^{d}$, there exists $\hat{\mathbf{z}} \in \mathbb{R}^{\hat{d}}$ such that $\hat{C} e^{\hat{A} t} \hat{\mathbf{z}}=C e^{A t} \mathbf{z}$ for all $t \in \mathbb{R}$. Suppose initially that $\hat{d} \leq d$. Since $\mathbf{z}$ is arbitrary, there must exist $T \in \mathbb{R}^{\hat{d} \times d}$ such that $\hat{C} \hat{A}^{k} T=C A^{k}$ $(k=0,1, \ldots)$. In particular, $V_{o}=\hat{V}_{o} T$. As $(C, A)$ and $(\hat{C}, \hat{A})$ are observable, then $V_{o}$ and $\hat{V}_{o}$ have full column rank, so $\hat{d}=d$ and $T=\left(\hat{V}_{o}^{T} \hat{V}_{o}\right)^{-1} \hat{V}_{o}^{T} V_{o}$, which is nonsingular (with $\left.T^{-1}=\left(V_{o}^{T} V_{o}\right)^{-1} V_{o}^{T} \hat{V}_{o}\right)$. In particular, $\hat{C}=C T^{-1}$. Also, since $V_{o} A=\hat{V}_{o} \hat{A} T$, then $\hat{A}=\left(\hat{V}_{o}^{T} \hat{V}_{o}\right)^{-1} \hat{V}_{o}^{T} V_{o} A T^{-1}=$ $T A T^{-1}$. Finally, from the variation of the constants formula (2)-(3), we require $V_{o} B=\hat{V}_{o} \hat{B}$, so $\hat{B}=T B$. A similar argument applies when $\hat{d} \geq d$, and completes the proof. 


\section{APPENDIX B}

\section{STORAGE FUNCTIONS}

The storage function concept features in many classical proofs of the positive-real lemma, e.g., [1], [2]. Here, in contrast to [1], [2], we present results on storage functions without any controllability assumptions.

We consider the following optimal control problem.

Definition B.1: Let $\mathcal{B}_{s}$ be as in (1); let $\sigma(\mathbf{u}, \mathbf{y}):=\mathbf{u}^{T} \Sigma_{11} \mathbf{u}+$ $2 \mathbf{u}^{T} \Sigma_{12} \mathbf{y}+\mathbf{y}^{T} \Sigma_{22} \mathbf{y}$ for some $\Sigma_{11} \in \mathbb{R}_{s}^{n \times n}, \Sigma_{12} \in \mathbb{R}^{n \times m}$ and $\Sigma_{22} \in \mathbb{R}_{s}^{m \times m}$; and, for any given $\mathbf{x}_{0} \in \mathbb{R}^{d}$, let

$$
\begin{array}{r}
\mathcal{E}_{+}^{\sigma}\left(\mathbf{x}_{0}\right)=\left\{\int_{t_{0}}^{t_{1}}-\sigma(\mathbf{u}, \mathbf{y})(t) d t \mid t_{1} \geq t_{0},(\mathbf{u}, \mathbf{y}, \mathbf{x}) \in \mathcal{B}_{s},\right. \\
\text { and } \left.\mathbf{x}\left(t_{0}\right)=\mathbf{x}_{0}\right\} .
\end{array}
$$

Then the available storage $S_{a}^{\sigma}$ with respect to the supply rate $\sigma$ satisfies (i) $S_{a}^{\sigma}\left(\mathbf{x}_{0}\right)=\sup \left(\mathcal{E}_{+}^{\sigma}\left(\mathbf{x}_{0}\right)\right)$ if $\mathcal{E}_{+}^{\sigma}\left(\mathbf{x}_{0}\right)$ is bounded above; and (ii) $S_{a}^{\sigma}\left(\mathbf{x}_{0}\right)=\infty$ otherwise.

Note, with $\Sigma_{11}=I, \Sigma_{12}=0$ and $\Sigma_{22}=-I$ (resp., $\Sigma_{11}=$ $\Sigma_{22}=0, \Sigma_{12}=\frac{1}{2} I$ ), then $S_{a}^{\sigma}=S_{a}^{\sigma_{g}}$ (resp., $S_{a}^{\sigma}=S_{a}^{\sigma_{p}}$ ). As in [1], we define a storage function with respect to $\sigma$ as follows.

Definition B.2: Let $\mathcal{B}_{s}$ be as in (1), and let $\sigma$ be as in Definition B.1. We say $S$ is a storage function with respect to the supply rate $\sigma$ if (i) $S\left(\mathbf{x}_{0}\right) \in \mathbb{R}$ and $S\left(\mathbf{x}_{0}\right) \geq 0$ for all $\mathbf{x}_{0} \in \mathbb{R}^{d}$; (ii) $S(0)=0$; and (iii) if $(\mathbf{u}, \mathbf{y}, \mathbf{x}) \in \mathcal{B}_{s}$ and $t_{1} \geq t_{0} \in \mathbb{R}$, then $S\left(\mathbf{x}\left(t_{1}\right)\right) \leq \int_{t_{0}}^{t_{1}}(\sigma(\mathbf{u}, \mathbf{y}))(t) d t+S\left(\mathbf{x}\left(t_{0}\right)\right)$.

The next lemma proves that the boundedness of the available storage is equivalent to the existence of a storage function.

Lemma B.3: Let $\mathcal{B}_{s}$ be as in (1); and let $\sigma$ and $S_{a}^{\sigma}$ be as in Definition B.1. The following hold:

1. If $S_{a}^{\sigma}\left(\mathbf{x}_{0}\right)<\infty$ for all $\mathrm{x}_{0} \in \mathbb{R}^{d}$, then $S_{a}^{\sigma}$ is a storage function with respect to $\sigma$.

2. If there exists a storage function with respect to $\sigma$ (denoted $S)$, then $S_{a}^{\sigma}\left(\mathbf{x}_{0}\right) \leq S\left(\mathbf{x}_{0}\right)<\infty$ for all $\mathbf{x}_{0} \in \mathbb{R}^{d}$.

Proof: See [1, Theorem 1].

In the next lemma, we prove that the available storage $S_{a}^{\sigma}\left(\mathbf{x}_{0}\right)$ is a quadratic form in $\mathbf{x}_{0}$, under an assumption which is satisfied by both passive and non-expansive systems.

Lemma B.4: Let $\mathcal{B}_{s}$ be as in (1); and let $\sigma$ and $S_{a}^{\sigma}$ be as in Definition B.1. Also, for any given $\mathbf{z} \in \mathbb{R}^{d}$ and $t_{0} \in \mathbb{R}$, let there exist $t_{1} \geq t_{0}$, and $(\mathbf{u}, \mathbf{y}, \mathbf{x}) \in \mathcal{B}_{s}$ with $\mathbf{x}\left(t_{0}\right)=\mathbf{z}$, such that $-\int_{t_{0}}^{t_{1}}(\sigma(\mathbf{u}, \mathbf{y}))(t) d t \geq 0$. If $S_{a}^{\sigma}\left(\mathbf{x}_{0}\right)<\infty$ for all $\mathbf{x}_{0} \in \mathbb{R}^{d}$, then there exists $X \in \mathbb{R}_{s}^{d \times d}$ with $X \geq 0$ such that $S_{a}^{\sigma}\left(\mathbf{x}_{0}\right)=\mathbf{x}_{0}^{T} X \mathbf{x}_{0}$ for all $\mathbf{x}_{0} \in \mathbb{R}^{d}$.

Proof: For any given $\mathbf{x}_{1}, \mathbf{x}_{2} \in \mathbb{R}^{d}$, we let $W\left(\mathbf{x}_{1}, \mathbf{x}_{2}\right):=$ $\frac{1}{4}\left(S_{a}^{\sigma}\left(\mathbf{x}_{1}+\mathbf{x}_{2}\right)-S_{a}^{\sigma}\left(\mathbf{x}_{1}-\mathbf{x}_{2}\right)\right)$. We then let $\mathbf{e}_{j}$ denote the $j$ th column of the identity matrix $I_{d}$, we let the $i j$ th entry of $X$ be defined as $X_{i j}:=W\left(\mathbf{e}_{i}, \mathbf{e}_{j}\right)(i, j=1, \ldots, d)$, and we will show that $X$ is symmetric and $S_{a}^{\sigma}(\mathbf{z})=\mathbf{z}^{T} X \mathbf{z}$ for all $\mathbf{z} \in \mathbb{R}^{d}$. To prove this, we will show that, for any given $\mathbf{x}_{1}, \mathbf{x}_{2} \in \mathbb{R}^{d}$ and $\lambda \in \mathbb{R}$, then

(i) $W\left(\lambda \mathbf{x}_{1}, \mathbf{x}_{2}\right)=\lambda W\left(\mathbf{x}_{1}, \mathbf{x}_{2}\right)$; and

(ii) $S_{a}^{\sigma}\left(\mathbf{x}_{1}+\mathbf{x}_{2}\right)+S_{a}^{\sigma}\left(\mathbf{x}_{1}-\mathbf{x}_{2}\right)=2\left(S_{a}^{\sigma}\left(\mathbf{x}_{1}\right)+S_{a}^{\sigma}\left(\mathbf{x}_{2}\right)\right)$.

From [19, Lemma 3], condition (ii) implies that, for any given $\mathbf{x}_{1}, \mathbf{x}_{2}, \mathbf{z} \in \mathbb{R}^{d}$, then (iia) $W\left(\mathbf{x}_{1}, \mathbf{x}_{2}\right)=W\left(\mathbf{x}_{2}, \mathbf{x}_{1}\right)$; (iib) $W\left(\mathbf{x}_{1}+\mathbf{x}_{2}, \mathbf{z}\right)=W\left(\mathbf{x}_{1}, \mathbf{z}\right)+W\left(\mathbf{x}_{2}, \mathbf{z}\right)$; and (iic) $S_{a}^{\sigma}(\mathbf{z})=$ $W(\mathbf{z}, \mathbf{z})$. Together with condition (i), we conclude that $W$ is a symmetric bilinear form, and $X$ is symmetric. We then let $\mathbf{z} \in \mathbb{R}^{d}$ and we denote the $i$ th entry of $\mathbf{z}$ by $z_{i}$, and it follows that $S_{a}^{\sigma}(\mathbf{z})=S_{a}^{\sigma}\left(\sum_{i=1}^{d} z_{i} \mathbf{e}_{i}\right)=W\left(\sum_{i=1}^{d} z_{i} \mathbf{e}_{i}, \sum_{j=1}^{d} z_{j} \mathbf{e}_{j}\right)=$ $\sum_{i=1}^{d} \sum_{j=1}^{d} z_{i} W\left(\mathbf{e}_{i}, \mathbf{e}_{j}\right) z_{j}=\sum_{i=1}^{d} \sum_{j=1}^{d} z_{i} X_{i j} z_{j}=\mathbf{z}^{T} X \mathbf{z}$.

It remains to show conditions (i) and (ii). We first show that, for any given $t_{1} \geq t_{0}$,

$$
\begin{aligned}
S_{a}^{\sigma}\left(\mathbf{x}_{0}\right)= & \sup _{\mathbf{u} \in \mathcal{L}_{2}^{\text {loc }}\left(\mathbb{R}, \mathbb{R}^{n}\right), t_{2} \geq t_{1}} \int_{t_{0}}^{t_{2}}-(\sigma(\mathbf{u}, \mathbf{y}))(t) d t, \\
& \text { such that }(\mathbf{u}, \mathbf{y}, \mathbf{x}) \in \mathcal{B}_{s}, \mathbf{x}\left(t_{0}\right)=\mathbf{x}_{0} .
\end{aligned}
$$

To see this, let $t_{1} \geq t_{0}$ and $(\mathbf{u}, \mathbf{y}, \mathbf{x}) \in \mathcal{B}_{s}$ with $\mathbf{x}\left(t_{0}\right)=\mathbf{x}_{0}$ satisfy $\int_{t_{0}}^{t_{1}}-(\sigma(\mathbf{u}, \mathbf{y}))(t) d t=S_{a}^{\sigma}\left(\mathbf{x}_{0}\right)-\epsilon$ for some $\epsilon>0$. Then, from the conditions in the lemma statement, there exist $t_{2} \geq t_{1}$ and $(\tilde{\mathbf{u}}, \tilde{\mathbf{y}}, \tilde{\mathbf{x}}) \in \mathcal{B}_{s}$ such that $\tilde{\mathbf{u}}(t)=\mathbf{u}(t), \tilde{\mathbf{y}}(t)=\mathbf{y}(t)$, and $\tilde{\mathbf{x}}(t)=\mathbf{x}(t)$ for all $t_{0} \leq t \leq t_{1}$; and $\int_{t_{1}}^{t_{2}}-(\sigma(\tilde{\mathbf{u}}, \tilde{\mathbf{y}}))(t) d t \geq 0$. It follows that $\int_{t_{0}}^{t}-(\sigma(\tilde{\mathbf{u}}, \tilde{\mathbf{y}}))(t) d t \geq S_{a}^{\sigma}\left(\mathbf{x}_{0}\right)-\epsilon$ for all $t \geq t_{1}$. But $S_{a}^{\sigma}\left(\mathbf{x}_{0}\right) \geq \int_{t_{0}}^{t}-(\sigma(\tilde{\mathbf{u}}, \tilde{\mathbf{y}}))(t) d t$, and $\epsilon>0$ can be made arbitrarily small by choosing $t_{1}$ and $\mathbf{u}$. This proves (35).

To prove (i), we let $\mathbf{x}_{1}, \mathbf{x}_{2} \in \mathbb{R}^{d}$ and $\lambda \in \mathbb{R}$ be fixed but arbitrary, and we show that $S_{a}^{\sigma}\left(\lambda \mathbf{x}_{1}+\mathbf{x}_{2}\right)+\lambda S_{a}^{\sigma}\left(\mathbf{x}_{1}-\mathbf{x}_{2}\right) \leq$ $S_{a}^{\sigma}\left(\lambda \mathbf{x}_{1}-\mathbf{x}_{2}\right)+\lambda S_{a}^{\sigma}\left(\mathbf{x}_{1}+\mathbf{x}_{2}\right)$. To see this, suppose instead that there exists $\epsilon>0$ such that

$$
\begin{aligned}
S_{a}^{\sigma}\left(\lambda \mathbf{x}_{1}+\mathbf{x}_{2}\right)+\lambda & S_{a}^{\sigma}\left(\mathbf{x}_{1}-\mathbf{x}_{2}\right) \\
& =S_{a}^{\sigma}\left(\lambda \mathbf{x}_{1}-\mathbf{x}_{2}\right)+\lambda S_{a}^{\sigma}\left(\mathbf{x}_{1}+\mathbf{x}_{2}\right)+\epsilon .
\end{aligned}
$$

There exist $\left(\mathbf{u}_{a}, \mathbf{y}_{a}, \mathbf{x}_{a}\right) \in \mathcal{B}_{s}$ with $\mathbf{x}_{a}\left(t_{0}\right)=\lambda \mathbf{x}_{1}+\mathbf{x}_{2}$, $\left(\mathbf{u}_{b}, \mathbf{y}_{b}, \mathbf{x}_{b}\right) \in \mathcal{B}_{s}$ with $\mathbf{x}_{b}\left(t_{0}\right)=\mathbf{x}_{1}-\mathbf{x}_{2}$, and $t_{1} \geq t_{0}$, with

$$
\begin{aligned}
& S_{a}^{\sigma}\left(\lambda \mathbf{x}_{1}+\mathbf{x}_{2}\right)+\lambda S_{a}^{\sigma}\left(\mathbf{x}_{1}-\mathbf{x}_{2}\right) \\
& \quad \leq \int_{t_{0}}^{t_{1}}\left(-\sigma\left(\mathbf{u}_{a}, \mathbf{y}_{a}\right)-\lambda\left(\sigma\left(\mathbf{u}_{b}, \mathbf{y}_{b}\right)\right)\right)(t)+\epsilon / 2 .
\end{aligned}
$$

Now, let $\left(\mathbf{u}_{c}, \mathbf{y}_{c}, \mathbf{x}_{c}\right):=(\lambda-1) /(1+\lambda)\left(\mathbf{u}_{a}, \mathbf{y}_{a}, \mathbf{x}_{a}\right)+2 \lambda /(1+$ $\lambda)\left(\mathbf{u}_{b}, \mathbf{y}_{b}, \mathbf{x}_{b}\right)$ and $\left(\mathbf{u}_{d}, \mathbf{y}_{d}, \mathbf{x}_{d}\right):=2 /(1+\lambda)\left(\mathbf{u}_{a}, \mathbf{y}_{a}, \mathbf{x}_{a}\right)+$ $(1-\lambda) /(1+\lambda)\left(\mathbf{u}_{b}, \mathbf{y}_{b}, \mathbf{x}_{b}\right)$. It can be verified that $\sigma\left(\mathbf{u}_{a}, \mathbf{y}_{a}\right)+$ $\lambda \sigma\left(\mathbf{u}_{b}, \mathbf{y}_{b}\right)=\sigma\left(\mathbf{u}_{c}, \mathbf{y}_{c}\right)+\lambda \sigma\left(\mathbf{u}_{d}, \mathbf{y}_{d}\right), \mathbf{x}_{c}\left(t_{0}\right)=\lambda \mathbf{x}_{1}-\mathbf{x}_{2}$ and $\mathbf{x}_{d}\left(t_{0}\right)=\mathbf{x}_{1}+\mathbf{x}_{2}$. It follows from (36)-(37) that

$$
\begin{aligned}
S_{a}^{\sigma}\left(\lambda \mathbf{x}_{1}+\right. & \left.\mathbf{x}_{2}\right)+\lambda S_{a}^{\sigma}\left(\mathbf{x}_{1}-\mathbf{x}_{2}\right) \\
& \leq \int_{t_{0}}^{t_{1}}\left(-\sigma\left(\mathbf{u}_{c}, \mathbf{y}_{c}\right)-\lambda\left(\sigma\left(\mathbf{u}_{d}, \mathbf{y}_{d}\right)\right)\right)(t)+\epsilon / 2 \\
& \leq S_{a}^{\sigma}\left(\lambda \mathbf{x}_{1}-\mathbf{x}_{2}\right)+\lambda S_{a}^{\sigma}\left(\mathbf{x}_{1}+\mathbf{x}_{2}\right)+\epsilon / 2 \\
& =S_{a}^{\sigma}\left(\lambda \mathbf{x}_{1}+\mathbf{x}_{2}\right)+\lambda S_{a}^{\sigma}\left(\mathbf{x}_{1}-\mathbf{x}_{2}\right)-\epsilon / 2,
\end{aligned}
$$

a contradiction. Substituting $-\mathbf{x}_{2}$ for $\mathbf{x}_{2}$ in the above argument gives $S_{a}^{\sigma}\left(\lambda \mathbf{x}_{1}-\mathbf{x}_{2}\right)+\lambda S_{a}^{\sigma}\left(\mathbf{x}_{1}+\mathbf{x}_{2}\right) \leq$ $S_{a}^{\sigma}\left(\lambda \mathbf{x}_{1}+\mathbf{x}_{2}\right)+\lambda S_{a}^{\sigma}\left(\mathbf{x}_{1}-\mathbf{x}_{2}\right)$, and completes the proof of (i).

To see (ii), suppose instead that there exists $\epsilon>0$ such that

$$
S_{a}^{\sigma}\left(\mathbf{x}_{1}+\mathbf{x}_{2}\right)+S_{a}^{\sigma}\left(\mathbf{x}_{1}-\mathbf{x}_{2}\right)+\epsilon=2\left(S_{a}^{\sigma}\left(\mathbf{x}_{1}\right)+S_{a}^{\sigma}\left(\mathbf{x}_{2}\right)\right) .
$$

Let $t_{1} \geq t_{0}$ and $\left(\mathbf{u}_{a}, \mathbf{y}_{a}, \mathbf{x}_{a}\right),\left(\mathbf{u}_{b}, \mathbf{y}_{b}, \mathbf{x}_{b}\right) \in \mathcal{B}_{s}$ with $\mathbf{x}_{a}\left(t_{0}\right)=\mathbf{x}_{1}$ and $\mathbf{x}_{b}\left(t_{0}\right)=\mathbf{x}_{2}$ be such that

$$
\begin{aligned}
& \int_{t_{0}}^{t_{1}}-\left(\sigma\left(\mathbf{u}_{a}, \mathbf{y}_{a}\right)\right)(t) d t+\frac{\epsilon}{8}>S_{a}^{\sigma}\left(\mathbf{x}_{1}\right), \text { and } \\
& \int_{t_{0}}^{t_{1}}-\left(\sigma\left(\mathbf{u}_{b}, \mathbf{y}_{b}\right)\right)(t) d t+\frac{\epsilon}{8}>S_{a}^{\sigma}\left(\mathbf{x}_{2}\right) .
\end{aligned}
$$

Similar to [19, p. 796], we let $\left(\tilde{\mathbf{u}}_{a}, \tilde{\mathbf{y}}_{a}, \tilde{\mathbf{x}}_{a}\right)=\left(\mathbf{u}_{a}, \mathbf{y}_{a}, \mathbf{x}_{a}\right)+$ $\left(\mathbf{u}_{b}, \mathbf{y}_{b}, \mathbf{x}_{b}\right)$ and $\left(\tilde{\mathbf{u}}_{b}, \tilde{\mathbf{y}}_{b}, \tilde{\mathbf{x}}_{b}\right)=\left(\mathbf{u}_{a}, \mathbf{y}_{a}, \mathbf{x}_{a}\right)-\left(\mathbf{u}_{b}, \mathbf{y}_{b}, \mathbf{x}_{b}\right)$. 
Then $\left(\tilde{\mathbf{u}}_{a}, \tilde{\mathbf{y}}_{a}, \tilde{\mathbf{x}}_{a}\right),\left(\tilde{\mathbf{u}}_{b}, \tilde{\mathbf{y}}_{b}, \tilde{\mathbf{x}}_{b}\right) \in \mathcal{B}_{s}, \tilde{\mathbf{x}}_{a}\left(t_{0}\right)=\mathbf{x}_{1}+\mathbf{x}_{2}$, and $\tilde{\mathbf{x}}_{b}\left(t_{0}\right)=\mathbf{x}_{1}-\mathbf{x}_{2}$, whence

$$
\begin{aligned}
& S_{a}^{\sigma}\left(\mathbf{x}_{1}+\mathbf{x}_{2}\right)+S_{a}^{\sigma}\left(\mathbf{x}_{1}-\mathbf{x}_{2}\right) \\
& \quad \geq \int_{t_{0}}^{t_{1}}-\left(\sigma\left(\tilde{\mathbf{u}}_{a}, \tilde{\mathbf{y}}_{a}\right)\right)(t) d t+\int_{t_{0}}^{t_{1}}-\left(\sigma\left(\tilde{\mathbf{u}}_{b}, \tilde{\mathbf{y}}_{b}\right)\right)(t) d t \\
& \quad=2\left(\int_{t_{0}}^{t_{1}}-\left(\sigma\left(\mathbf{u}_{a}, \mathbf{y}_{a}\right)\right)(t) d t+\int_{t_{0}}^{t_{1}}-\left(\sigma\left(\mathbf{u}_{b}, \mathbf{y}_{b}\right)\right)(t) d t\right) \\
& \quad>2\left(S_{a}^{\sigma}\left(\mathbf{x}_{1}\right)+S_{a}^{\sigma}\left(\mathbf{x}_{2}\right)-\frac{\epsilon}{4}\right)=S_{a}^{\sigma}\left(\mathbf{x}_{1}+\mathbf{x}_{2}\right)+S_{a}^{\sigma}\left(\mathbf{x}_{1}-\mathbf{x}_{2}\right)+\frac{\epsilon}{2},
\end{aligned}
$$

a contradiction. Thus, $S_{a}^{\sigma}\left(\mathbf{x}_{1}+\mathbf{x}_{2}\right)+S_{a}^{\sigma}\left(\mathbf{x}_{1}-\mathbf{x}_{2}\right) \leq$ $2\left(S_{a}^{\sigma}\left(\mathbf{x}_{1}\right)+S_{a}^{\sigma}\left(\mathbf{x}_{2}\right)\right)$. A similar argument shows that $S_{a}^{\sigma}\left(\mathbf{x}_{1}+\mathbf{x}_{2}\right)+S_{a}^{\sigma}\left(\mathbf{x}_{1}-\mathbf{x}_{2}\right) \geq 2\left(S_{a}^{\sigma}\left(\mathbf{x}_{1}\right)+S_{a}^{\sigma}\left(\mathbf{x}_{2}\right)\right)$, and completes the proof of (ii).

We next consider a related optimal control problem concerning the observer staircase form in note Al.1.

Lemma B.5: Let $\mathcal{B}_{s}$ be as in (1); let $\sigma$ and $S_{a}^{\sigma}$ be as in Definition B.1, let $S_{a}^{\sigma}\left(\mathbf{x}_{0}\right)<\infty$ for all $\mathbf{x}_{0} \in \mathbb{R}^{d}$; let $T_{1}$ and $\hat{\mathcal{B}}_{s}$ be as in note Al1, and let

$$
\begin{aligned}
\hat{S}_{a}^{\sigma}\left(\hat{\mathbf{x}}_{0}\right):= & \sup _{t_{1} \geq t_{0}, \mathbf{u} \in \mathcal{L}_{2}^{\text {Ioc }}\left(\mathbb{R}, \mathbb{R}^{n}\right)} \int_{t_{0}}^{t_{1}}-(\sigma(\mathbf{u}, \mathbf{y}))(t) d t, \\
& \text { such that }(\mathbf{u}, \mathbf{y}, \hat{\mathbf{x}}) \in \hat{\mathcal{B}}_{s}, \hat{\mathbf{x}}\left(t_{0}\right)=\hat{\mathbf{x}}_{0} .
\end{aligned}
$$

Then $S_{a}^{\sigma}\left(\mathbf{x}_{0}\right)=\hat{S}_{a}^{\sigma}\left(T_{1} \mathbf{x}_{0}\right)$ for all $\mathbf{x}_{0} \in \mathbb{R}^{d}$. In particular, with $V_{o}$ as in (6), then $\mathbf{z} \in \mathbb{R}^{d}$ and $V_{o} \mathbf{z}=0$ imply $S_{a}^{\sigma}(\mathbf{z})=0$.

Proof: Let $T=\operatorname{col}\left(T_{1} T_{2}\right)$ be as in note A|1. It can be shown from the variation of the constants formula (2)-(3) that (i) if $(\mathbf{u}, \mathbf{y}, \mathbf{x}) \in \mathcal{B}_{s}$ satisfies $\mathbf{x}\left(t_{0}\right)=\mathbf{x}_{0}$, then there exists $(\mathbf{u}, \mathbf{y}, \hat{\mathbf{x}}) \in \hat{\mathcal{B}}_{s}$ with $\hat{\mathbf{x}}\left(t_{0}\right)=T_{1} \mathbf{x}_{0}$; and (ii) if $(\mathbf{u}, \mathbf{y}, \hat{\mathbf{x}}) \in$ $\hat{\mathcal{B}}_{s}$ satisfies $\hat{\mathbf{x}}\left(t_{0}\right)=\hat{\mathbf{x}}_{0}$, and $\hat{\mathbf{x}}_{1} \in \mathbb{R}^{d-\hat{d}}$, then there exists $(\mathbf{u}, \mathbf{y}, \mathbf{x}) \in \mathcal{B}_{s}$ with $\mathbf{x}\left(t_{0}\right)=T^{-1} \operatorname{col}\left(\begin{array}{ll}\hat{\mathbf{x}}_{0} & \hat{\mathbf{x}}_{1}\end{array}\right)$. Now, consider a fixed but arbitrary $\mathbf{x}_{0} \in \mathbb{R}^{d}$. It follows from (i) that

$$
\begin{aligned}
S_{a}^{\sigma}\left(\mathbf{x}_{0}\right) \leq \sup _{t_{1} \geq t_{0}, \mathbf{u} \in \mathcal{L}_{2}^{\text {loc }}\left(\mathbb{R}, \mathbb{R}^{n}\right)} \int_{t_{0}}^{t_{1}}-(\sigma(\mathbf{u}, \mathbf{y}))(t) d t, \\
\quad \text { such that }(\mathbf{u}, \mathbf{y}, \hat{\mathbf{x}}) \in \hat{\mathcal{B}}_{s}, \hat{\mathbf{x}}\left(t_{0}\right)=T_{1} \mathbf{x}_{0},
\end{aligned}
$$

i.e., $S_{a}^{\sigma}\left(\mathbf{x}_{0}\right) \leq \hat{S}_{a}^{\sigma}\left(T_{1} \mathbf{x}_{0}\right)$. Similarly, from (ii), it can be shown that $S_{a}^{\sigma}\left(\mathbf{x}_{0}\right) \geq \hat{S}_{a}^{\sigma}\left(T_{1} \mathbf{x}_{0}\right)$, so $S_{a}^{\sigma}\left(\mathbf{x}_{0}\right)=\hat{S}_{a}^{\sigma}\left(T_{1} \mathbf{x}_{0}\right)$. Finally, if $V_{o} \mathbf{z}=0$, then it can be shown that $T_{1} \mathbf{z}=0$. As $\hat{S}_{a}^{\sigma}$ is a storage function by Lemma B.3, then $S_{a}^{\sigma}(\mathbf{z})=\hat{S}_{a}^{\sigma}(0)=0$.

\section{APPENDIX C}

\section{POSITIVE-REAL AND BOUNDED-REAL PAIRS}

Here, we provide several results relating to the new concepts of positive-real and bounded-real pairs.

C]1 Let $P \in \mathbb{R}^{m \times n}[\xi]$ and $Q \in \mathbb{R}^{m \times m}[\xi]$; and let

$$
\begin{aligned}
& J_{n}:=\frac{1}{2}\left[\begin{array}{cc}
0 & I_{n} \\
I_{n} & 0
\end{array}\right], \Sigma_{m, n}:=\left[\begin{array}{cc}
I_{n} & 0 \\
0 & -I_{m}
\end{array}\right], \\
& \Psi(\eta, \xi):=\left[\begin{array}{ll}
P & -Q
\end{array}\right](\eta) \Sigma_{m, n}\left[\begin{array}{ll}
P & -Q
\end{array}\right](\xi)^{T} \text {, and } \\
& \Phi(\eta, \xi):=\left[\begin{array}{ll}
P & -Q
\end{array}\right](\eta) J_{n}\left[\begin{array}{ll}
P & -Q
\end{array}\right](\xi)^{T} \text { if } m=n \text {. }
\end{aligned}
$$

Then $(P, Q)$ is a positive-real pair (resp., bounded-real pair) if and only if (i) $\Phi(\lambda, \bar{\lambda}) \leq 0$ (resp., $\Psi(\lambda, \bar{\lambda}) \leq 0$ ) for all $\lambda \in \mathbb{C}_{+}$; (ii) $\operatorname{rank}\left(\left[\begin{array}{ll}P & -Q\end{array}\right](\lambda)\right)=n$ for all $\lambda \in \overline{\mathbb{C}}_{+}$; and (iii) if $\mathbf{p} \in \mathbb{R}^{n}[\xi]$ and $\lambda \in \mathbb{C}$ satisfy $\mathbf{p}(\xi)^{T} \Phi(\xi,-\xi)=0$ (resp., $\mathbf{p}(\xi)^{T} \Psi(\xi,-\xi)=0$ ) and $\mathbf{p}(\lambda)^{T}[P-Q](\lambda)=0$, then $\mathbf{p}(\lambda)=0$.
C]2 Let $P, Q \in \mathbb{R}^{n \times n}[\xi]$; let $J_{n}$ be as in (39); let $Y \in$ $\mathbb{R}^{n \times n}[\xi]$ and $S \in \mathbb{R}^{2 n \times 2 n}$ be nonsingular with $S J_{n} S^{T}=J_{n}$;

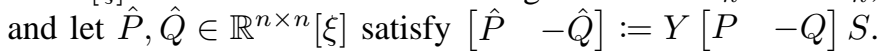
Then $(P, Q)$ is a positive-real pair if and only if $(\hat{P}, \hat{Q})$ is a positive-real pair (this follows from note C11).

C] 3 Let $P, Q \in \mathbb{R}^{n \times n}[\xi]$; let $J_{n}$ and $\Sigma_{n, n}$ be as in (39); let $Y \in \mathbb{R}^{n \times n}[\xi]$ and $S \in \mathbb{R}^{2 n \times 2 n}$ be nonsingular with $S J_{n} S^{T}=\Sigma_{n, n}$; and let $\hat{P}, \hat{Q} \in \mathbb{R}^{n \times n}[\xi]$ satisfy $[\hat{P}-\hat{Q}]:=$ $Y\left[\begin{array}{ll}P & -Q\end{array}\right] S$. Then $(P, Q)$ is a bounded-real pair if and only if $(\hat{P}, \hat{Q})$ is a positive-real pair (this follows from note C.11).

C. 4 Let $\Sigma \in \mathbb{R}^{n \times n}$ be a signature matrix (i.e., $\Sigma$ is diagonal with diagonal entries \pm 1 ), let $P, Q \in \mathbb{R}^{n \times n}[\xi]$, and let $\hat{Q}:=\frac{1}{2}(Q-P \Sigma)$ and $\hat{P}:=\frac{1}{2}(P \Sigma+Q)$. Then $(P, Q)$ is a bounded-real pair if and only if $(\hat{P}, \hat{Q})$ is a positive-real pair (this follows from note Cl3). Also, if $P, Q \in \mathbb{R}^{n \times n}[\xi]$ and $Q^{-1} P$ is proper, then there necessarily exists a signature matrix $\Sigma$ and matrices $\hat{Q}:=\frac{1}{2}(Q-P \Sigma)$ and $\hat{P}:=\frac{1}{2}(P \Sigma+Q)$ such that $\hat{Q}$ is nonsingular and $\hat{Q}^{-1} \hat{P}$ is proper. To obtain such matrices $\Sigma, \hat{P}$ and $\hat{Q}$, we let $\tilde{P}:=\frac{1}{2}(P+Q)$ and $\tilde{Q}:=\frac{1}{2}(Q-P)$, so $P=\tilde{P}-\tilde{Q}$ and $Q=\tilde{P}+\tilde{Q}$. We then let $S_{1}$ and $S_{2} \in \mathbb{R}^{n \times n}$ be matrices that select columns from $\tilde{P}$ and $\tilde{Q}$ to achieve the maximal determinantal degree. I.e., (i) $S_{1}$ and $S_{2}$ are diagonal matrices with all entries either 0 or 1; (ii) $S_{1}+S_{2}=I$; and (iii) $\operatorname{deg}\left(\operatorname{det}\left(\tilde{P} S_{1}+\tilde{Q} S_{2}\right)\right)$ takes its maximum value among all matrices $S_{1}$ and $S_{2}$ that satisfy (i) and (ii). We then let $\hat{P}:=\tilde{P} S_{2}+\tilde{Q} S_{1}, \hat{Q}:=\tilde{P} S_{1}+\tilde{Q} S_{2}$, and $\Sigma:=S_{2}-S_{1}$, so $\Sigma$ is a signature matrix. The method in [5. Proof of Theorem 9] then proves that $\hat{Q}^{-1} \hat{P}$ is proper.

\section{APPENDIX D \\ EXPLICIT CHARACTERISATION OF THE AVAILABLE ENERGY: SUPPLEMENTARY LEMMAS}

In this final appendix, we present four supplementary lemmas used in the proof of Theorem 13

Lemma D.1: Let $\mathcal{B}_{s}$ and $H$ be as in (1) and (8) with $m=n$; let $\operatorname{spec}(A) \in \overline{\mathbb{C}}_{-}$; let $X_{-}, L$ and $W$ be real matrices that satisfy condition 2(iiia) of Theorem 13, and let

$$
Z(\xi):=W+L(\xi I-A)^{-1} B \text { and } Y(\xi):=\left[\begin{array}{cc}
\xi I-A & -B \\
L & W
\end{array}\right] .
$$

Then $Z^{\star} Z=H+H^{\star}$, and $Z$ is a spectral factor for $H+H^{\star}$ if and only if $Y(\lambda)$ has full row rank for all $\lambda \in \mathbb{C}_{+}$.

Proof: That $Z^{\star} Z=H+H^{\star}$ follows by pre-multiplying $\Omega(X)$ in (7) by $\left[B^{T}\left(-\xi I-A^{T}\right)^{-1} \quad I\right]$ and post-multiplying by $\operatorname{col}\left((\xi I-A)^{-1} B \quad I\right)$. Since $\operatorname{spec}(A) \in \overline{\mathbb{C}}_{-}$, then $Z$ is analytic in $\mathbb{C}_{+}$. Finally, consider a fixed but arbitrary $\lambda \in \mathbb{C}_{+}$, so $\lambda I-A$ is nonsingular. It remains to show that $Z(\lambda)$ has full row rank if and only if $Y(\lambda)$ does. This follows from

$$
\left[\begin{array}{cc}
0 & \lambda I-A \\
Z(\lambda) & L
\end{array}\right]=\left[\begin{array}{cc}
\lambda I-A & -B \\
L & W
\end{array}\right]\left[\begin{array}{cc}
(\lambda I-A)^{-1} B & I \\
I & 0
\end{array}\right],
$$

since the rightmost matrix in this equation is nonsingular.

The final three lemmas relate to the decomposition in case (ii) in the proof of Theorem 13 We refer back to that proof for definitions of conditions (R1) (R4) and (S1) + (S4).

Lemma D.2: Let $P_{k-1}, Q_{k-1}$ satisfy (R1) for $i=k-1$, and let $D_{k-1}:=\lim _{\xi \rightarrow \infty}\left(Q_{k-1}^{-1} P_{k-1}(\xi)\right)$. The following hold. 
1. Let $P_{k}:=P_{k-1}-\frac{1}{2} Q_{k-1}\left(D_{k-1}-D_{k-1}^{T}\right)$ and $Q_{k}:=Q_{k-1}$. Then (R1) and (R2) hold for $i=k$.

2. Let $A_{k}, B_{k}, C_{k}, D_{k}$ satisfy (S1) for $i=k$; and let $A_{k-1}:=A_{k}, B_{k-1}:=B_{k}$, and $C_{k-1}:=C_{k}$. Then:

a) (S1) holds for $i=k-1$.

b) Let $X_{k-1}, L_{k-1}, W_{k-1}, X_{k}, L_{k}$, and $W_{k}$ be real matrices with $X_{k}=X_{k-1} \geq 0, L_{k}=L_{k-1}$, and $W_{k}=W_{k-1}$. Then (i) (S3) holds for $i=k-1$ if and only if (S3) holds for $i=k$; and (ii) (S4) holds for $i=k-1$ if and only if (S4) holds for $i=k$.

Proof: Condition 11 Clearly, $Q_{k}^{-1} P_{k}=$ $Q_{k-1}^{-1} P_{k-1}-\frac{1}{2}\left(D_{k-1}-D_{k-1}^{T}\right)$, so $Q_{k}^{-1} P_{k}$ is proper and $\lim _{\xi \rightarrow \infty}\left(Q_{k}^{-1} P_{k}(\xi)\right)=\lim _{\xi \rightarrow \infty}\left(Q_{k-1}^{-1} P_{k-1}(\xi)\right)-$ $\frac{1}{2}\left(D_{k-1}-D_{k-1}^{T}\right)=\frac{1}{2}\left(D_{k-1}+D_{k-1}^{T}\right)$. Next, let

$$
S=\left[\begin{array}{cc}
I & 0 \\
\frac{1}{2}\left(D_{k-1}-D_{k-1}^{T}\right) & I
\end{array}\right] .
$$

Then $\left[\begin{array}{ll}P_{k} & -Q_{k}\end{array}\right]=\left[\begin{array}{ll}P_{k-1} & -Q_{k-1}\end{array}\right] S$, and it follows from note C/2 that $\left(P_{k}, Q_{k}\right)$ is a positive-real pair.

Condition 2. Let $\mathcal{A}_{k}, M_{k}, N_{k}, U_{k}, V_{k}, E_{k}, F_{k}$ be as in (S1) for the case $i=k$; and let $E_{k-1}:=E_{k}-\frac{1}{2} F_{k}\left(D_{k-1}-\right.$ $\left.D_{k-1}^{T}\right), F_{k-1}:=F_{k}, M_{k-1}:=M_{k}, N_{k-1}:=N_{k}, U_{k-1}:=$ $U_{k}$, and $V_{k-1}:=V_{k}$. By post-multiplying both sides of the relationship in (S1) for the case $i=k$ by $\operatorname{diag}\left(S^{-1} I\right)$, we find that (S1) holds for $i=k-1$. Finally, condition $2 \mathrm{~b}$ follows since $D_{k}+D_{k}^{T}=D_{k-1}+D_{k-1}^{T}$. Thus, with $\Omega_{i}\left(X_{i}\right)$ as in (S3), then $\Omega_{k}\left(X_{k}\right)=\Omega_{k-1}\left(X_{k-1}\right)$.

Lemma D.3: Let $P_{k-1}, Q_{k-1}$ satisfy (R1) (R2) for $i=k-1$, and let $n_{k}:=\operatorname{normalrank}\left(P_{k-1}\right), m_{k}:=n_{k-1}-n_{k}$, and $r_{k}:=\operatorname{rank}\left(D_{k-1}\right)$. The following hold.

1. There exists a nonsingular $T \in \mathbb{R}^{n_{k-1} \times n_{k-1}}$; unimodular $Y \in \mathbb{R}^{n_{k-1} \times n_{k-1}}[\xi]$ and $\tilde{Q}_{22} \in \mathbb{R}^{m_{k} \times m_{k}}[\xi] ; \tilde{Q}_{12} \in$ $\mathbb{R}^{n_{k} \times m_{k}}[\xi]$; and $P_{k}, Q_{k}$ satisfying (R1) (R3) for $i=k$, with

$$
Y P_{k-1} T=\left[\begin{array}{cc}
P_{k} & 0 \\
0 & 0
\end{array}\right], Y Q_{k-1}\left(T^{-1}\right)^{T}=\left[\begin{array}{cc}
Q_{k} & \tilde{Q}_{12} \\
0 & \tilde{Q}_{22}
\end{array}\right] .
$$

2. Let $A_{k}, B_{k}, C_{k}, D_{k}$ satisfy (S1) for $i=k$; and $A_{k-1}:=A_{k}$, $B_{k-1}:=\left[\begin{array}{ll}B_{k} & 0\end{array}\right] T^{-1}, C_{k-1}:=\left(T^{-1}\right)^{T} \operatorname{col}\left(\begin{array}{ll}C_{k} & 0\end{array}\right)$, and $D_{k-1}:=\left(T^{-1}\right)^{T} \operatorname{diag}\left(\begin{array}{ll}D_{k} & 0\end{array}\right) T^{-1}$. Then:

a) (S1) holds for $i=k-1$.

b) Let $X_{k-1}, L_{k-1}$ and $W_{k-1}$ satisfy (S3) for $i=k-1$; partition $T$ as $T=:\left[\begin{array}{ll}T_{1} & T_{2}\end{array}\right]$ with $T_{1} \in \mathbb{R}^{n_{k-1} \times n_{k}}$ and $T_{2} \in \mathbb{R}^{n_{k-1} \times m_{k}}$; and let $X_{k}:=X_{k-1}, L_{k}:=L_{k-1}$, and $W_{k}:=W_{k-1} T_{1}$. Then (i) $W_{k-1} T_{2}=0$; (ii) (S3) holds for $i=k$; and (iii) if (S4) holds for $i=k-1$, then (S4) holds for $i=k$.

c) Let $X_{k}, L_{k}$ and $W_{k}$ satisfy (S3) for $i=k$; and let $X_{k-1}:=X_{k}, L_{k-1}:=L_{k}$, and $W_{k-1}:=\left[\begin{array}{ll}W_{k} & 0\end{array}\right] T^{-1}$. Then (i) (S3) holds for $i=k-1$; and (ii) if (S4) holds for $i=k$, then [S4) holds for $i=k-1$.

Proof: Condition 11 Since $\left(P_{k-1}, Q_{k-1}\right)$ is a positivereal pair and $Q_{k-1}$ is nonsingular, then $H:=Q_{k-1}^{-1} P_{k-1}$ is positive-real (see Remark 9]. Since, in addition, $D_{k-1}$ is symmetric, then $D_{k-1} \geq 0$ by Theorem 10, so there exists $T_{1 a} \in \mathbb{R}^{n_{k-1} \times r_{k}}$ such that $T_{1 a}^{T} D_{k-1} T_{1 a}=I_{r_{k}}$ by Sylvester's law of inertia (as $\left.\operatorname{rank}\left(D_{k-1}\right)=r_{k}\right)$. Now, let the columns of $T_{2} \in \mathbb{R}^{n_{k-1} \times m_{k}}$ be a basis for the nullspace of
$H$ (i.e., $T_{2}$ has full column rank and $H T_{2}=0$ ). Then, since the nullspace of $H$ is contained in the nullspace of $D_{k-1}$, there exists $T_{1 b} \in \mathbb{R}^{n_{k-1} \times\left(n_{k}-r_{k}\right)}$ such that the columns of $\left[\begin{array}{ll}T_{1 b} & T_{2}\end{array}\right]$ are a basis for the nullspace of $D_{k-1}$. With $T_{1}=\left[\begin{array}{ll}T_{1 a} & T_{1 b}\end{array}\right]$, then $T=\left[\begin{array}{ll}T_{1} & T_{2}\end{array}\right]$ is nonsingular and $T^{T} D_{k-1} T=\operatorname{diag}\left(I_{r_{k}} \quad 0\right)$. Also, from [3, Theorem 8.4.1], $T^{T} H T=\operatorname{diag}\left(\begin{array}{cc}\hat{H} & 0\end{array}\right)$, where $\hat{H} \in \mathbb{R}^{n_{k} \times n_{k}}(\xi)$ is positivereal and nonsingular.

From [7, Theorem B.1.1], there exists a unimodular $Y \in$ $\mathbb{R}^{n_{k-1} \times n_{k-1}}[\xi]$ such that $\tilde{Q}:=Y Q_{k-1}\left(T^{-1}\right)^{T}$ is upper triangular. Let $\tilde{P}:=Y P_{k-1} T$, and note that $\tilde{Q}$ is nonsingular with $\tilde{Q}^{-1} \tilde{P}=T^{T} H T=\operatorname{diag}\left(\begin{array}{ll}\hat{H} & 0\end{array}\right)$. Since $\tilde{P}=\tilde{Q} \operatorname{diag}\left(\begin{array}{ll}\hat{H} & 0\end{array}\right)$, then $\tilde{P}$ and $\tilde{Q}$ (partitioned compatibly with $\left.\operatorname{diag}\left(\begin{array}{ll}\hat{H} & 0\end{array}\right)\right)$ take the form indicated in (42). To show that $\tilde{Q}_{22}$ in (42) is unimodular, we let $\lambda \in \mathbb{C}$ and $\hat{\mathbf{p}} \in \mathbb{R}^{m_{k}}[\xi]$ satisfy $\hat{\mathbf{p}}(\lambda)^{T} \tilde{Q}_{22}(\lambda)=0$, and $\mathbf{p}^{T}:=\left[\begin{array}{ll}0 & \hat{\mathbf{p}}^{T}\end{array}\right] Y$. It can be verified that $\mathbf{p}^{T}\left(P_{k-1} Q_{k-1}^{\star}+\right.$ $\left.Q_{k-1} P_{k-1}^{\star}\right)=0$ and $\mathbf{p}(\lambda)^{T}\left[\begin{array}{ll}P_{k-1} & -Q_{k-1}\end{array}\right](\lambda)=0$. Since $\left(P_{k-1}, Q_{k-1}\right)$ is a positive-real pair, this implies $\mathbf{p}(\lambda)=0$. Since, in addition, $Y$ is unimodular, then $\hat{\mathbf{p}}(\lambda)=0$, and it follows that $\tilde{Q}_{22}$ is unimodular. It is then easily shown from notes C[1 and $\mathrm{Cl} 2$ that $\left(P_{k}, Q_{k}\right)$ is a positive-real pair. Moreover, $T^{T} H T=T^{T} Q_{k-1}^{-1} P_{k-1} T=\operatorname{diag}\left(Q_{k}^{-1} P_{k} \quad 0\right)=$ $\operatorname{diag}\left(\begin{array}{ll}\hat{H} & 0\end{array}\right)$ where $\hat{H}$ is nonsingular and $\lim _{\xi \rightarrow \infty}(\hat{H}(\xi))=$ $T^{T} D_{k-1} T=\operatorname{diag}\left(I_{r_{k}} \quad 0\right)$. Thus, $P_{k}, Q_{k}$ satisfy (R1) (R3) for $i=k$.

Condition 2, Let $\mathcal{A}_{k}, M_{k}, N_{k}, U_{k}, V_{k}, E_{k}, F_{k}$ be as in (S1) for the case $i=k$; and let

$$
\left[\begin{array}{cc}
M_{k-1} & N_{k-1} \\
U_{k-1} & V_{k-1}
\end{array}\right]:=\left[\begin{array}{cc}
Y^{-1} & 0 \\
0 & I
\end{array}\right]\left[\begin{array}{cc|c}
M_{k} & \tilde{Q}_{12} & N_{k} \\
0 & Q_{22} & 0 \\
\hline U_{k} & 0 & V_{k}
\end{array}\right]\left[\begin{array}{cc}
T^{T} & 0 \\
0 & I
\end{array}\right] .
$$

It can be verified that each of these four matrices is unimodular. Also, with $\mathcal{A}_{i}$ as in (S1) for $i=k-1$ and $i=k$, then

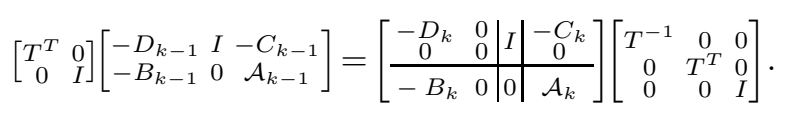

Thus, with $E_{k-1}:=\left[\begin{array}{ll}E_{k} & 0\end{array}\right] T^{-1}$ and $F_{k-1}:=\left[\begin{array}{ll}F_{k} & 0\end{array}\right] T^{T}$, it can be verified that (S1) holds for $i=k-1$. To see 2b note initially that $T_{2}^{T} W_{k-1}^{T} W_{k-1} T_{2}=T_{2}^{T}\left(D_{k-1}+D_{k-1}^{T}\right) T_{2}=0$, so $W_{k-1} T_{2}=0$. Next, note that

$$
\left[\begin{array}{c|cc}
\xi I-A_{k} & -B_{k} & 0 \\
L_{k} & W_{k} & 0
\end{array}\right]=\left[\begin{array}{cc}
\xi I-A_{k-1} & -B_{k-1} \\
L_{k-1} & W_{k-1}
\end{array}\right]\left[\begin{array}{cc}
I & 0 \\
0 & T
\end{array}\right] .
$$

We denote the rightmost matrix in (43) by $S$; we let $\Omega_{k-1}\left(X_{k-1}\right)$ and $\Omega_{k}\left(X_{k}\right)$ be as in (S3), and we note that $S^{T} \Omega_{k-1}\left(X_{k-1}\right) S=\operatorname{diag}\left(\Omega_{k}\left(X_{k}\right) 0\right)$. This shows $2 \mathrm{~b}$ (ii). Also, since $S$ is nonsingular, then $2 \mathrm{~b}$ (iii) holds. The proof of 2c is similar, noting that (43) also holds in this case.

Lemma D.4: Let $P_{k-1}, Q_{k-1}$ satisfy (R1) (R3) for $i=k-1$, with $m_{k}:=n_{k-1}-r_{k-1}>0$. The following hold.

1. There exists $0<K \in \mathbb{R}^{m_{k} \times m_{k}}$ such that $\lim _{\xi \rightarrow \infty}\left(\frac{1}{\xi} P_{k-1}^{-1} Q_{k-1}(\xi)\right)=\operatorname{diag}(0 \quad K)$.

2. Let $P_{k}(\xi):=Q_{k-1}(\xi)-P_{k-1}(\xi) \operatorname{diag}(0 \quad K \xi)$, and $Q_{k}:=P_{k-1}$. Then (R1) holds for $i=k$; $\operatorname{deg}\left(\operatorname{det}\left(Q_{k}\right)\right)<\operatorname{deg}\left(\operatorname{det}\left(Q_{k-1}\right)\right)$; and there exist $\hat{D}_{12} \in$ $\mathbb{R}^{r_{k-1} \times m_{k}}, \hat{D}_{21} \in \mathbb{R}^{m_{k} \times r_{k-1}}, \hat{D}_{22} \in \mathbb{R}^{m_{k} \times m_{k}}$ such that

$$
\lim _{\xi \rightarrow \infty}\left(Q_{k}^{-1} P_{k}(\xi)\right)=: D_{k}=\left[\begin{array}{cc}
I_{r_{k-1}} & \hat{D}_{12} \\
\hat{D}_{21} & \hat{D}_{22}
\end{array}\right] .
$$


3. Let $A_{k}, B_{k}, C_{k}, D_{k}$ satisfy [S1) for $i=k$; partition $B_{k}, C_{k}$ compatibly with $D_{k}$ as $B_{k}=\left[\begin{array}{ll}\hat{B}_{1} & \hat{B}_{2}\end{array}\right], C_{k}=$ $\operatorname{col}\left(\begin{array}{ll}\hat{C}_{1} & \hat{C}_{2}\end{array}\right)$; and let

$$
\begin{aligned}
A_{k-1} & :=\left[\begin{array}{cc}
A_{k}-\hat{B}_{1} \hat{C}_{1} & \hat{B}_{2} K^{-1}-\hat{B}_{1} \hat{D}_{12} K^{-1} \\
\hat{D}_{21} \hat{C}_{1}-\hat{C}_{2} & \hat{D}_{21} \hat{D}_{12} K^{-1}-\hat{D}_{22} K^{-1}
\end{array}\right], \\
B_{k-1} & :=\left[\begin{array}{cc}
\hat{B}_{1} & 0 \\
-\hat{D}_{21} & I
\end{array}\right], \text { and } C_{k-1}:=\left[\begin{array}{cc}
-\hat{C}_{1} & -\hat{D}_{12} K^{-1} \\
0 & K^{-1}
\end{array}\right] .
\end{aligned}
$$

Then:

a) (S1) holds for $i=k-1$.

b) Let $X_{k-1}, L_{k-1}$ and $W_{k-1}$ satisfy (S3) for $i=$ $k-1$; partition $L_{k-1}$ and $W_{k-1}$ compatibly with $A_{k-1}, B_{k-1}, C_{k-1}$ and $D_{k-1}$ as $L_{k-1}:=\left[\begin{array}{cc}\tilde{L}_{1} & \tilde{L}_{2}\end{array}\right]$ and $W_{k-1}:=\left[\begin{array}{ll}\tilde{W}_{1} & \tilde{W}_{2}\end{array}\right] ;$ and let $L_{k}:=\tilde{L}_{1}+\tilde{W}_{1} \hat{C}_{1}$, and $W_{k}:=\left[\begin{array}{ll}\tilde{W}_{1} & \tilde{L}_{2} K+\tilde{W}_{1} \hat{D}_{12}\end{array}\right]$. Then (i) $\tilde{W}_{2}=0$; (ii)

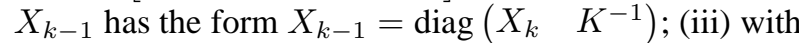
$X_{k}$ as in condition (ii), then (S3) holds for $i=k$; and (iv) if (S4) holds for $i=k-1$, then (S4) holds for $i=k$.

c) Let $X_{k}, L_{k}$ and $W_{k}$ satisfy (S3)] for $i=k$; partition $W_{k}$ compatibly with $D_{k}$ as $W_{k}=\left[\begin{array}{ll}\hat{W}_{1} & \hat{W}_{2}\end{array}\right]$; and let $L_{k-1}:=\left[\begin{array}{ll}L_{k}-\hat{W}_{1} \hat{C}_{1} & \left(\hat{W}_{2}-\hat{W}_{1} \hat{D}_{12}\right) K^{-1}\end{array}\right]$, $W_{k-1}:=\left[\begin{array}{ll}\hat{W}_{1} & 0\end{array}\right]$ and $X_{k-1}:=\operatorname{diag}\left(\begin{array}{ll}X_{k} & K^{-1}\end{array}\right)$. Then (i) (S3) holds for $i=k-1$; and (ii) if (S4) holds for $i=k$, then (S4) holds for $i=k-1$.

Proof: Condition 1 Since $\left(P_{k-1}, Q_{k-1}\right)$ is a positivereal pair and $P_{k-1}$ is nonsingular, then $P_{k-1}^{-1} Q_{k-1}$ is positivereal. Hence, if $P_{k-1}^{-1} Q_{k-1}$ has a pole at infinity, then it is simple and the residue matrix $J:=\lim _{\xi \rightarrow \infty}\left(\frac{1}{\xi}\left(P_{k-1}^{-1} Q_{k-1}\right)(\xi)\right)$ is real and non-negative definite [3, Theorem 2.7.2]. Thus, there exist real matrices $J, \hat{D}$ and strictly proper real-rational matrices $G, H$ (partitioned compatibly with $D_{k-1}$ ) such that

$$
\begin{aligned}
& P_{k-1}^{-1} Q_{k-1}(\xi)=\left[\begin{array}{cc}
J_{11} & J_{12} \\
J_{12}^{T} & J_{22}
\end{array}\right] \xi+\left[\begin{array}{ll}
\hat{D}_{11} & \hat{D}_{12} \\
\hat{D}_{21} & \hat{D}_{22}
\end{array}\right]+\left[\begin{array}{ll}
G_{11} & G_{12} \\
G_{21} & G_{22}
\end{array}\right](\xi) \\
& Q_{k-1}^{-1} P_{k-1}(\xi)=\left[\begin{array}{cc}
I_{r_{k}-1} & 0 \\
0 & 0
\end{array}\right]+\left[\begin{array}{ll}
H_{11} & H_{12} \\
H_{21} & H_{22}
\end{array}\right](\xi) .
\end{aligned}
$$

By considering the first block row in the equation $0=$ $\lim _{\xi \rightarrow \infty}\left(\frac{1}{\xi}\left(Q_{k-1}^{-1} P_{k-1} P_{k-1}^{-1} Q_{k-1}\right)(\xi)\right)$, we obtain $J_{11}=0$ and $J_{12}=0$. Then, by considering the bottom right block in the equation $\lim _{\xi \rightarrow \infty}\left(\left(P_{k-1}^{-1} Q_{k-1} Q_{k-1}^{-1} P_{k-1}\right)(\xi)\right)=I$, we find that $J_{22} \lim _{\xi \rightarrow \infty}\left(\xi H_{22}(\xi)\right)=I$, which implies that $J_{22}$ is nonsingular. By letting $K:=J_{22}$, we obtain condition 1 .

Condition 2. Since $Q_{k}^{-1} P_{k}(\xi)=P_{k-1}^{-1} Q_{k-1}(\xi)-$ $\operatorname{diag}\left(\begin{array}{ll}0 & K \xi\end{array}\right)$, then $Q_{k}^{-1} P_{k}$ is proper and positive-real by [3. Theorem 8.4.3], and $\lim _{\xi \rightarrow \infty}\left(\left(Q_{k}^{-1} P_{k}\right)(\xi)\right)$ is equal to the matrix $\hat{D}$ in (45). Then, the top left block in the equation $I=\lim _{\xi \rightarrow \infty}\left(\left(P_{k-1}^{-1} Q_{k-1} Q_{k-1}^{-1} P_{k-1}\right)(\xi)\right)$ gives $\hat{D}_{11}=I_{r_{k-1}}$. Next, note that $\left[\begin{array}{ll}P_{k} & -Q_{k}\end{array}\right]=\left[\begin{array}{ll}P_{k-1} & -Q_{k-1}\end{array}\right] S$ where

$$
S=\left[\begin{array}{cc}
S_{11} & -I \\
-I & 0
\end{array}\right], \text { with } S_{11}(\xi)=\left[\begin{array}{cc}
0 & 0 \\
0 & -K \xi
\end{array}\right] .
$$

With $J_{n}$ as defined in Appendix $\mathrm{C}$, it can be verified that $S$ is unimodular and $S J_{n} S^{\star}=J_{n}$, and it is then easily shown that $\left(P_{k}, Q_{k}\right)$ satisfy conditions (b) and (c) in Definition 8 Since, in addition, $Q_{k}^{-1} P_{k}$ is positive-real, then $\left(P_{k}, Q_{k}\right)$ also satisfies condition (a) in Definition 8 , so $\left(P_{k}, Q_{k}\right)$ is a positivereal pair.
Finally, that $\operatorname{deg}\left(\operatorname{det}\left(Q_{k}\right)\right)<\operatorname{deg}\left(\operatorname{det}\left(Q_{k-1}\right)\right)$ will follow from condition 3a, noting from the final two block columns in (S1) that $\operatorname{deg}\left(\operatorname{det}\left(Q_{i}\right)\right)=\operatorname{deg}\left(\operatorname{det}\left(\mathcal{A}_{i}\right)\right)$.

Condition 3. Let $\mathcal{A}_{k}, M_{k}, N_{k}, U_{k}, V_{k}, E_{k}, F_{k}$ be as in (S1) for the case $i=k$, partition these matrices compatibly as

$$
\left[\begin{array}{ll|l}
\hat{M}_{11} & \hat{M}_{12} & \hat{N}_{1} \\
\hat{M}_{21} & \hat{M}_{22} & \hat{N}_{2} \\
\hline \hat{U}_{1} & \hat{U}_{2} & \hat{V}
\end{array}\right] \text {, and }\left[\begin{array}{cc|c|c}
-I & -\hat{D}_{12} & -\hat{C}_{1} \\
\hline-\hat{D}_{21} & -\hat{D}_{22} & -\hat{C}_{2} \\
\hline-\hat{B}_{1} & -\hat{B}_{2} & 0 & \mathcal{A}_{k}
\end{array}\right] \text {, }
$$

and let

$$
\left[\begin{array}{cc}
M_{k-1} & N_{k-1} \\
U_{k-1} & V_{k-1}
\end{array}\right]=\left[\begin{array}{cc|cc}
\hat{M}_{11} & \hat{M}_{12} & \hat{N}_{1} & 0 \\
\hat{M}_{21} & \hat{M}_{22} & \hat{N}_{2} & 0 \\
\hline-\hat{U}_{1} & \hat{U}_{2} & -\hat{V} & 0 \\
0 & I & 0 & -I
\end{array}\right]\left[\begin{array}{cc|cc}
I & \hat{D}_{12} & 0 & 0 \\
\hat{D}_{21} & \hat{D}_{22}+K \xi & 0 & I \\
\hline \hat{B}_{1} & \hat{B}_{2} & -I & 0 \\
\hat{D}_{21} & \hat{D}_{22}+K(1+\xi) & 0 & I
\end{array}\right] .
$$

It can be verified that each of the above matrices is unimodular (the modulus of the determinant of the rightmost matrix is equal to $\operatorname{det}(K))$. Also, with $E_{k-1}:=\operatorname{col}\left(\begin{array}{ll}F_{k} & 0) \text { and }\end{array}\right.$ $F_{k-1}(\xi):=\operatorname{col}\left(E_{k}(\xi) \quad 0\right)+\operatorname{col}\left(\xi \hat{U}_{2}(\xi) \quad I\right)\left[\begin{array}{ll}0 & K\end{array}\right]$, it can be verified that (S1) holds for $i=k-1$. Now, let $L_{k-1}, W_{k-1}$ be as in condition [3b. Since $W_{k-1}^{T} W_{k-1}=D+D^{T}=$ $\operatorname{diag}\left(2 I_{r_{k-1}} \quad 0\right)$, then $\tilde{W}_{2}^{T} \tilde{W}_{2}=0$, which proves $3 \mathrm{~b}$ (i). To show 3 b (ii), we partition $X_{k-1}$ compatibly with $A_{k-1}$ as

$$
X_{k-1}=\left[\begin{array}{cc}
X_{11} & X_{12} \\
X_{12}^{T} & X_{22}
\end{array}\right]
$$

Since $C_{k-1}^{T}-X_{k-1} B_{k-1}=L_{k-1}^{T} W_{k-1}=\left[\begin{array}{lll}L_{k-1}^{T} \tilde{W}_{1} & 0\end{array}\right]$, then $X_{12}=0$ and $X_{22}=K^{-1}$. Now, note that

$$
\left[\begin{array}{cc|cc}
\xi I-A_{k}-\hat{B}_{1} & -\hat{B}_{2} & 0 \\
0 & 0 & 0 & -I \\
\hline L_{k} & \hat{W}_{1} & \hat{W}_{2} & 0
\end{array}\right]=\left[\begin{array}{cc|cc}
\xi I-A_{k-1} & -B_{k-1} \\
L_{k-1} & W_{k-1}
\end{array}\right]\left[\begin{array}{ccc|cc}
I & 0 & 0 & 0 \\
0 & 0 & K & 0 \\
\hat{C}_{1} & I & \hat{D}_{12} & 0 \\
\hat{C}_{2} & \hat{D}_{21} & \xi K+\hat{D}_{22} & I
\end{array}\right] .
$$

We denote the rightmost matrix in this equation by $S$, we let $\Omega_{k-1}\left(X_{k-1}\right)$ and $\Omega_{k}\left(X_{k}\right)$ be as in (S3), and by direct calculation we obtain $S^{T} \Omega_{k-1}\left(X_{k-1}\right) S=\operatorname{diag}\left(\Omega_{k}\left(X_{k}\right) \quad 0\right)$. This proves $3 \mathrm{~b}$ (iii). Condition $3 \mathrm{~b}$ (iv) then follows since the rightmost matrix in the above displayed equation is nonsingular.

Next, let $X_{k}, L_{k}, W_{k}, X_{k-1}, L_{k-1}$ and $W_{k-1}$ be as in condition $3 \mathrm{c}$. We recall that the rightmost matrix in the above displayed equation (denoted $S$ ) is nonsingular. We then find that $\Omega_{k-1}\left(X_{k-1}\right)=\left(S^{-1}\right)^{T} \operatorname{diag}\left(\Omega_{k}\left(X_{k}\right)\right.$ 0) $S^{-1}$, so $3 \mathrm{c}$ (i) holds, and 3 c (ii) follows since $S^{-1}$ is nonsingular.

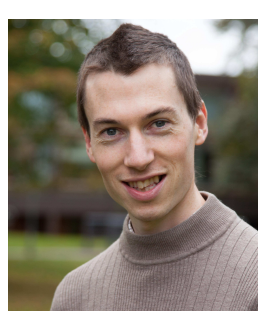

Timothy H. Hughes received the M.Eng. degree in mechanical engineering, and the Ph.D. degree in control engineering, from the University of Cambridge, U.K., in 2007 and 2014, respectively. From 2007 to 2010 he was employed as a mechanical engineer at The Technology Partnership, Hertfordshire, U.K; and from 2013 to 2017 he was a Henslow Research Fellow at the University of Cambridge, supported by the Cambridge Philosophical Society. $\mathrm{He}$ is currently a lecturer in the Department of Mathematics at the University of Exeter, U.K. 